\title{
Diets and Habitat Analyses of Mule Deer on the 200 Areas of The Hanford Site in Southcentral Washington
}

D. W. Uresk

V. A. Uresk

October 1980

Prepared for Rockwell Hanford Operations under Related Services Agreement with the U.S. Department of Energy Contract DE-AC06-76RLO 1830

Pacific Northwest Laboratory Operated for the U.S. Department of Energy by Battelle Memorial Institute 
NOTICE

This report was prepared as an account of work sponsored by the United States Government. Neither the United States nor the Department of Energy, nor any of their employees, nor any of their contractors, subcontractors, or their employees, makes any warranty, express or implied, or assumes any legal liability or responsibility for the accuracy, completeness or usefulness of any information, apparatus, product or process disclosed, or represents that its use would not infringe privately owned rights.

The views, opinions and conclusions contained in this report are those of the contractor and do not necessarily represent those of the United States Government or the United States Department of Energy.

\author{
PACIFIC NORTHWEST LABORATORY \\ operated by \\ BATTELLE \\ for the \\ UNITED STATES DEPARTMENT OF ENERGY \\ Under Contract EY-76-C-06-1830
}
Printed in the United States of America Available from
National Technical Information Service United States Department of Commerce 5285 Port Royal Road Springfield, Virginia 22151

Price: Printed Copy $\mathbf{S}$

-: Microfiche $\$ 3.00$

NTIS

$\begin{array}{lr}\text { *Pages } & \text { Selling Price } \\ 001-025 & \$ 4.00 \\ 026-050 & \$ 4.50 \\ 051-075 & \$ 5.25 \\ 076-100 & \$ 6.00 \\ 101-125 & \$ 6.50 \\ 126-150 & \$ 7.25 \\ 151-175 & \$ 8.00 \\ 176-200 & \$ 9.00 \\ 201-225 & \$ 9.25 \\ 226-250 & \$ 9.50 \\ 251-275 & \$ 10.75 \\ 276-300 & \$ 11.00\end{array}$




\section{7}

DIETS AND HABITAT ANALYSES OF MULE DEER ON THE 200 AREAS OF THE HANFORD SITE IN SOUTHCENTRAL WASHINGTON

D. W. Uresk*

V. A. Uresk ${ }^{* *}$

October 1980

*U.S. Forest Service Rocky Mounta in Forest and Range Experiment Station School of Mines Rapid City, South Dakota 57701

**Formerly with Rockwell Hanford Richland, Washington 99352

Prepared for Rockwell Hanford Operations under a Related Services Agreement with the U.S. Department of Energy under Contract DE-AC06-76RL0-1830

This research was performed as part of Rockwel1 Hanford Operations' Long-term Transuranic Defense Waste Program

Pacific Northwest Laboratory Richland, Washington 99352 


\section{ACKNOWLEDGEMENTS}

This study was supported by Rockwell Hanford Operations (RHO) as part of a program to document the potential for transporting waste radionuclides through biotic pathways. Many of the methods to analyze diet and vegetation were developed on the Arid Land Ecology Reserve under separate funding by the Department of Energy.

The authors wish to thank Marci Combs, Mark Rumble, Fern Nelson and Virgil Charles for their helpful suggestions and technical assistance during this study. Thanks are extended to Lee Rogers, Jack $\mathrm{Cl}$ ine and Donald Paine for their critical reviews and technical advice. Special thanks are given to Richard M. Hansen and Theresa Foppe at Colorado State University for dietary analyses of the deer pellets, and to Jerran T. Flinders at Brigham Young University who provided deer pellets from Deer Creek, Provo, Utah. 


\section{EXECUTIVE SUMMARY}

Forty-four food items were identified in the fecal pellets of the mule deer (Odocoileus hemionus hemionus) on three areas of the Hanford Site. Microscopic analysis of plant fragments indicated that bitterbrush was the most common species occurring in the diets of deer from the B-C Cribs area. Russian thistle (Salsola $\mathrm{kali}$ ) and goldenrod (Solidago sp.) were the most abundant plants found in the fecal pellets collected from B Pond and Gable Mountain Pond habitats, respectively. The similarity in diets among the habitats was low, ranging from $10 \%$ to $16 \%$. Preference indices of forage plants among sites were not similar (7\% to 19\%), indicating that deer are selecting different food plants at each site, even though many plants are common to all three sites.

The B-C Cribs, B Pond and Gable Mountain Pond habitats were characterized for canopy cover and frequency of occurrence of plant species. Twelve species were sampled in the B-C Cribs and B Pond areas; 22 species were identified on the Gable Mountain site. The most commonly occurring plant was cheatgrass (Bromus tectorum) in all three sites. The similarity in frequency and canopy cover of plants was low among sites, indicating that each site is different if these two parameters are considered.

Mule deer inhabiting the Hanford Site can serve as a pathway for movement of radioactive material from low-level radioactive waste management areas to man. Maximum levels of 137Cs found in deer pellet groups collected from $B$ Pond and Gable Mountain Pond areas were $100 \mathrm{pCi} / \mathrm{g}$ and $128 \mathrm{pCi} / \mathrm{g}$, respectively. Background levels were reported at B-C Cribs area. Maximum $90 \mathrm{Sr}$ values found in deer pellets at $B$ Pond were $107 \mathrm{pCi} / \mathrm{g}$ and $184 \mathrm{pCi} / \mathrm{g}$ at Gable Mountain Pond. These values indicate that mule deer are consuming radioactive materials through the forage plants at the B Pond and Gable Mountain Pond habitats. 


\section{CONTENTS}

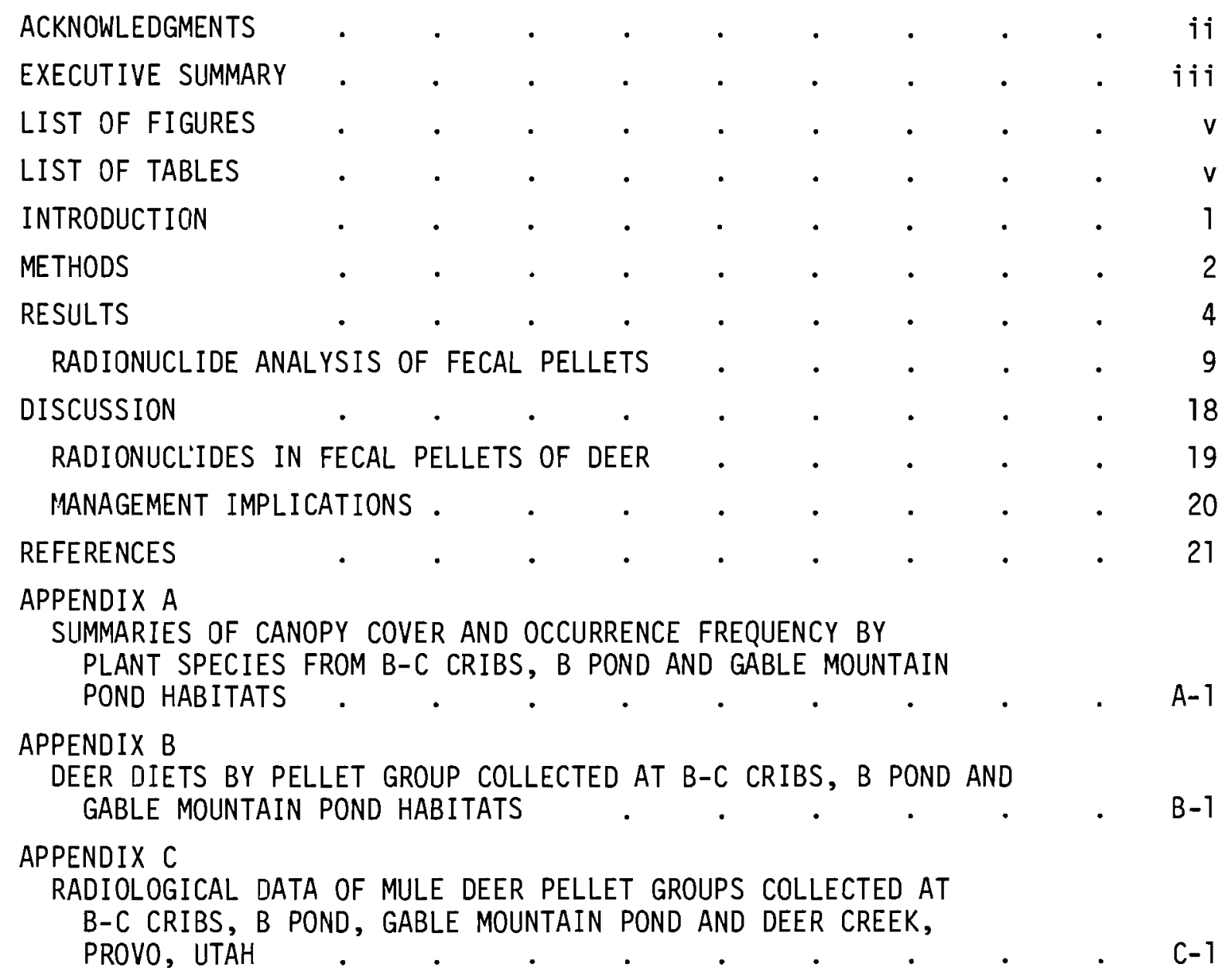




\section{FIGURES}

1. Map Showing the Location of the Three Study Areas, B-C Cribs, B Pund, and Gable Mountain Pond on the Hanford Site

2. Cesium-137 Found in Mule Deer Pellets from B-C Cribs, B Ponds, and Gable Mountain Pond Areas on the Hanford Site .

\section{TABLES}

1. Frequency of Occurrence and Canopy Cover of Plants

Within Three Habitat Sites Used by Mule Deer During

1977 and on the Hanford Site

2. Botanical Composition of Fecal Pellets Collected from Mule Deer During 1976 from Three Habitat Sites on the Hanford Site

3. Preference Indices of Mule Deer by Food Items on Three Areas of the Hanford Reservation

4. Average Similarity Indices (\%) and Rank-Order Correlation Coefficients $\left(r_{s}\right)$ of Mule Deer Diets, Preference Indices, Frequency of Occurrence and Canopy Cover When the Three Areas are Compared

5. Cerium-144 Content of Mule Deer Feces Collected from the 200 Area Plateau, and Deer Creek, Utah . . . . . . . . 12

6. Ruthenium-106 Content of Mule Deer Feces Collected from the 200 Area Plateau and Deer Creek, Utah . . . . . . . 12

7. Cesium-137 Content of Mule Deer Feces Collected from the 200 Area Plateau and Deer Creek, Utah . . . . . . . . 13

8. Zirconium-95 Content of Mule Deer Feces Collected from the 200 Area Plateau and Deer Creek, Utah . . . . . . . 13

9. Manganese-54 Content of Mule Deer Feces Collected from the 260 Area Plateau and Deer Creek, Utah . . . . . . . 15

10. Zinc-65 Content of Mule Deer Feces Collected from the 200 Area Plateau and Deer Creek, Utah . . . . . . . . . 15

11. Potassium-40 Content of Mule Deer Feces Collected from the 200 Area Plateau and Deer Creek, Utah, 
12. Cobalt-60 Content of Mule Deer Feces collected from the 200 Area Plateau and Deer Creek, Utah . . . .

13. Strontium-90 Analysis of Deer Pellet Groups Collected Adjacent to B Pond and Gable Mountain Pond on the Hanford Site During 1976 


\section{$\underline{\text { INTRODUCTION }}$}

On rangelands, knowledge of the diets of herbivores and the availability of forage plants aids in formulating better management programs for both plants and animals. The diets of mule deer (Odocoileus hemionus hemionus), a common heribivore on shrub-steppe rangeland of southcentral Washington, have been estimated by microhistological examination of fecal pellets. Food habits of the mule deer in eastern Oregon on bunchgrass rangeland have been reported by Edgerton and Smith (1971). Other studies of food habits of mule deer in bunchgrass rangelands have been reported by Nellis and Ross (1969), Lovaas (1958), Morris and Schwartz (1957), and Wilkins (1957).

The purpose of this study was to determine the dietary habits of mule deer at three locations on the 200 Area plateau of the Hanford Site in southcentral Washington. The study area has not been grazed by livestock since 1943, but small mammals such as the black-tailed jackrabbit (Lepus

californicus) and Townsend ground squirrel (Spermophilus townsendi) utilize the area. 
METHODS

The study area is located on the Department of Energy's (DOE) Hanford Site in southcentral Washington on a large plain which slopes gently towurd the Columbia River. This area is described as Artemesia tridentata/Poa sandbergii associations (Daubenmire, 1970). This community has been extensively invaded by alien annuals, especially cheatgrass (Bromus tectorum), which is the dominant understory species (Cline et al., 1977). The elevation is approximately $223 \mathrm{~m}$, and the area has an average annual rainfall of $16 \mathrm{~cm}$. The soils are primarily Rupert sands and Burbank loamy sand (Hajek, 1966). The topography is described as gently undulating.

Three study sites were selected, each differing in plant species composition. One site (Gable Mountain Pond) is located near a 71-acre man-made pond with dense riparian vegetation (Figure 1). The second study site (B Pond) is near a 45-acre man-made pond with sparse riparian vegetation. The third site (B-C Cribs area) is located in an area of sagebrush and Sandberg bluegrass. These study areas are near chemical separation and waste management operations associated with DOE's plutonium processing facilities.

Vegetation was analyzed for species composition, canopy cover by plant species, and frequency of occurrence. Four transects, adjacent and parallel to the shoreline of the ponds, were examined at each site. Transects sampled by Cline et al. (1977) in the B-C Cribs area were also used in this study. Canopy cover and frequency of occurrence were estimated by ocular examination at $50\left(2 \times 5 \mathrm{dm}, 0.1 \mathrm{~m}^{2}\right)$ plots, systematically spaced at $1-\mathrm{m}$ intervals along each 50-m line transect (Daubenmire, 1959). Canopy cover and frequency of occurrence values were summarized for each line transect. All data were collected during May 4-10, 1977.

Deer fecal pellets were collected along repeated transects located randomly within the study sites. At Gable Mountain Pond and B Pond, four transects were located adjacent and parallel to the shoreline of the ponds. The first 10 pellet groupings (recent, less than a month old and old, one month to several years) encountered along the transect were collected and individually placed in paper bags for dietary analysis. Forty pellet groups were collected at each of the pond sites. At the B-C Cribs site, two transects were randomly located within the radiation control area, and the first 10 pellet groups encountered on each transect were collected for dietary analysis. A total of 100 pellet groups was collected from all three study sites. The pellets were dried at $70{ }^{\circ} \mathrm{C}$ for 48 hours and ground through a wiley mill (1-mm screen) to make a homogeneous sample. All fecal pellet groups, both recent and old, were collected on either July 30 or August 4, 1976.

Five slides were prepared for each pellet group after the ground sample was washed over a 0.1-mm screen (Sparks and Malechek, 1968). The slides were prepared with Hertwig's solution and Hoyer's solution and dried for approximately $72 \mathrm{hr}$ at $60{ }^{\circ} \mathrm{C}$ (Baumgartner and Martin, 1939). 


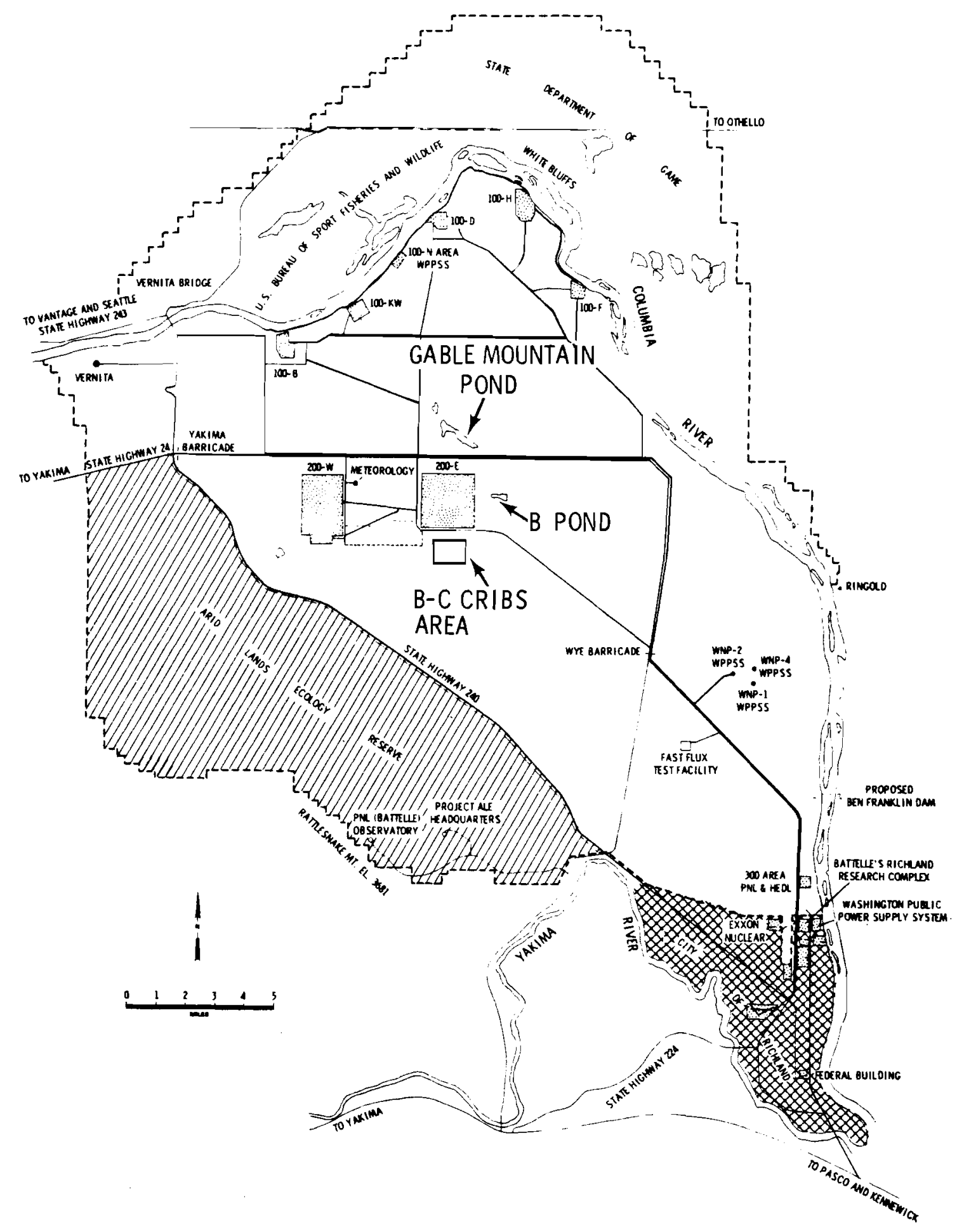

FIGURE 1. Map Showing the Location of the Three Study Areas, B-C Cribs, $B$ Pond, and Gable Mountain Pond on the Hanford Site. 
The slides were examined microscopically to identify the plant fragments on the basis of epidermal cell characteristics and were compared to reference plant material prepared from plant specimens identified and collected from the study areas. Twenty microscope fields were examined on each slide. All identifiable fragments were recorded, as were the unknowns. Each pellet group was individually analyzed for radionuclides by gamma energy analysis using a sodium iodide ( $\mathrm{NaI}$ ) crystal. Those pellet groups with detectable $137 \mathrm{Cs}$ were also analyzed for $90 \mathrm{Sr}$ by U.S. Testing Company, Richland, WA.

Statistical analyses follow Snedecor and Cochran (1967). Kulczynski's similarity indices (0osting, 1956) and Spearman's rank correlation coefficients ( $r_{s}$ ) (Siege1, 1956) were calculated for diet and plant communities to determine the degree of association.

Preference indices were calculated by dividing the relative frequency of a plant species in the diet by the relative frequency in the habitat. All values were rounded to the nearest one-tenth unit. Preference indices as used here are regarded only as a guide to food preferences rather than as absolute values.

\section{RESULTS}

\section{CHARACTERIZATION OF VEGETATION}

Frequency of occurrence and canopy cover provided by plants within three study areas are presented in Table 1. A total of 12 species was sampled in the B-C Cribs and B Pond areas; 22 species were present on the Gable Mountain site. The most cormmonly occurring plant at all three sites was cheatgrass (Bromus tectorum). Tansy mustard (Descurainea pinnata) and Russian thistle (Salsola kali) were the most common forbs present at the B-C Cribs and B Pond areas, respectively. Matted cryptantha (Cryptantha circumscissa) was the most common forb at the Gable Mountain Pond study area. Big sagebrush (Artemesia tridentata) was abundant in the B-C Cribs and B Pond areas, and hopsage (Atriplex spinosa) was the dominant shrub at the Gable Mountain Pond area.

Grasses and grass-1ike plants provided 17\%, 14\% and 6\% of the canopy cover on the Gable Mountain Pond, B Pond and B-C Cribs study areas, respectively (Table 1). Forbs contributed less canopy cover than the grasses and grasslikeplants. Gable Mountain study area had the largest forb canopy cover, $10 \%$. Forbs provided only $2 \%$ of the cover at the B-C Cribs area.

Shrubs dominated the three study areas, with the highest canopy cover occurring on the B-C Cribs site (17\%) and the least at Gable Mountain Pond $(5 \%)$. Sagebrush and hopsage were the major shrubs present on these areas. Canopy cover averaged $52 \%$ in the three areas; bare ground averaged approximately $48 \%$. Further analyses are presented in Appendix A.

\section{DEER DIETS}

Forty-four plants were identified in the fecal pellets of deer collected from the three areas (Table 2). Frequency of occurrence values show that 


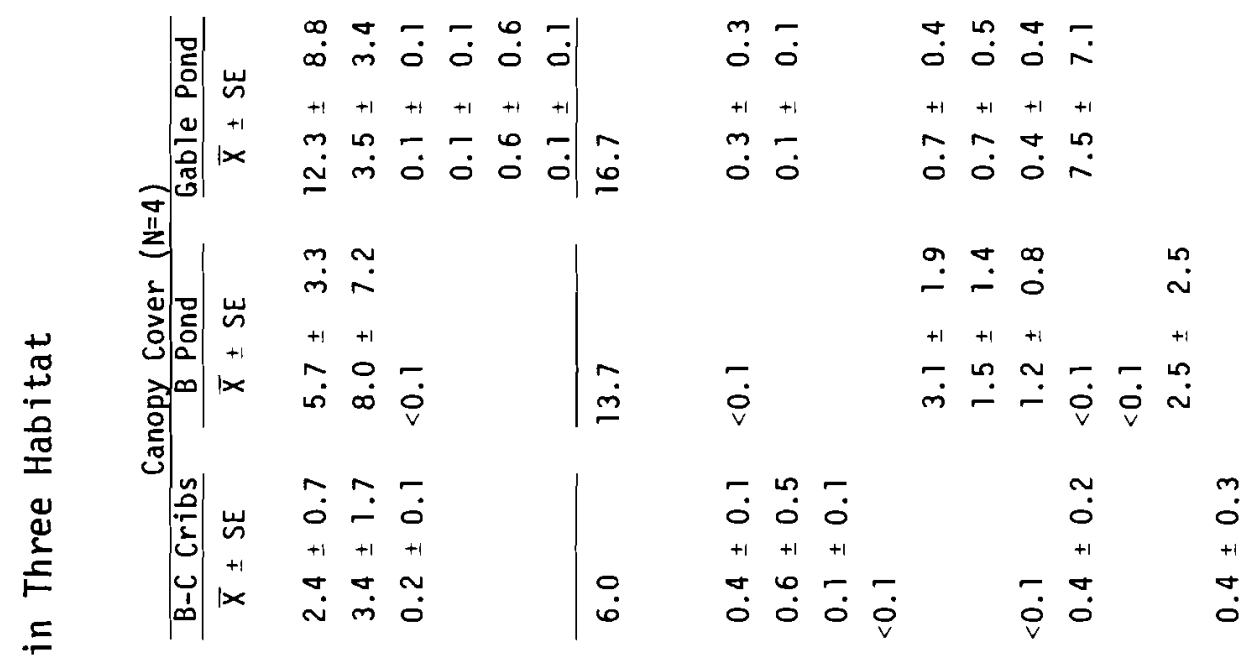

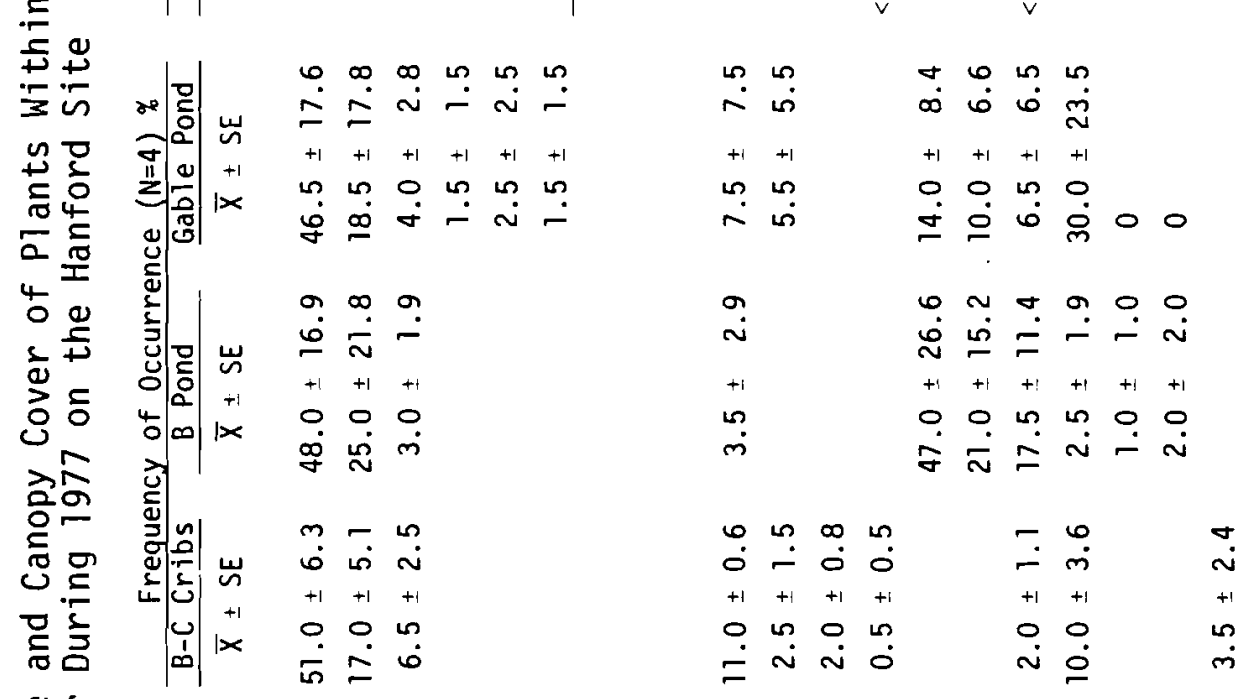

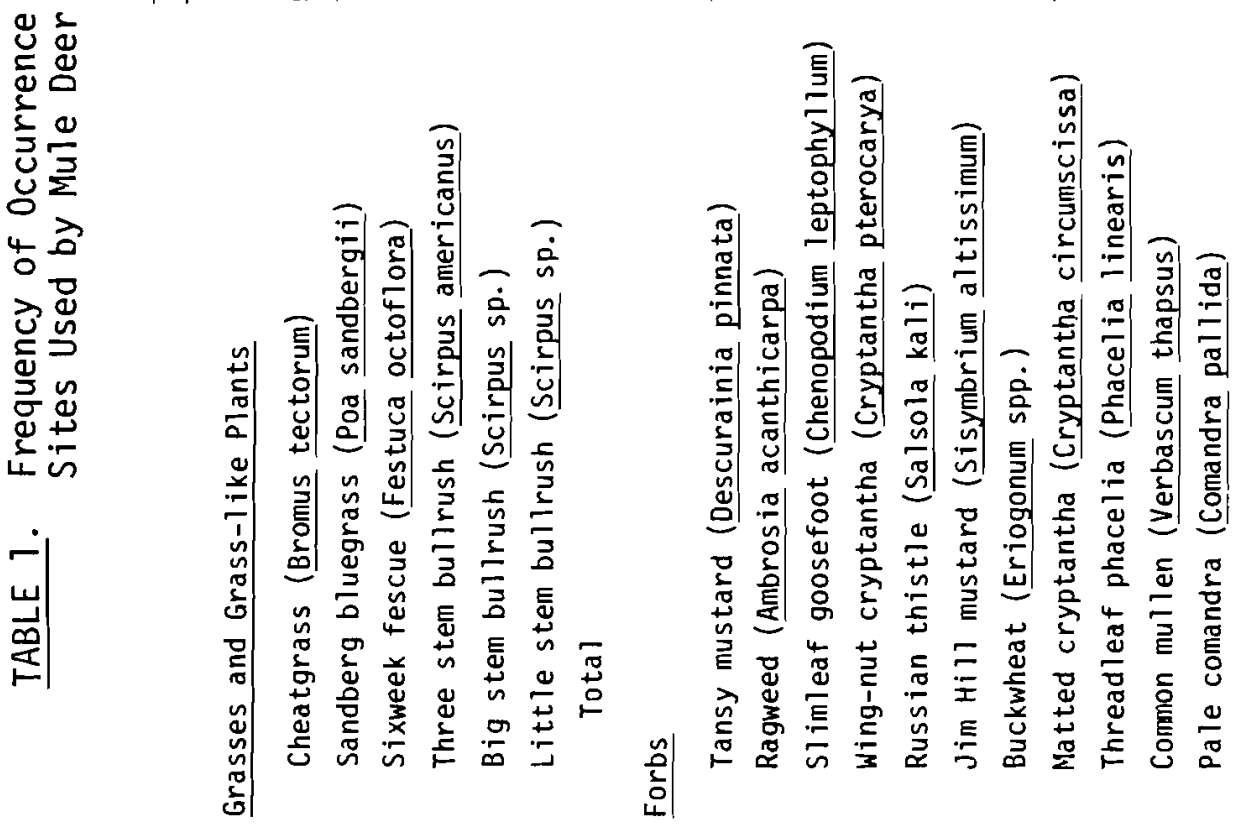




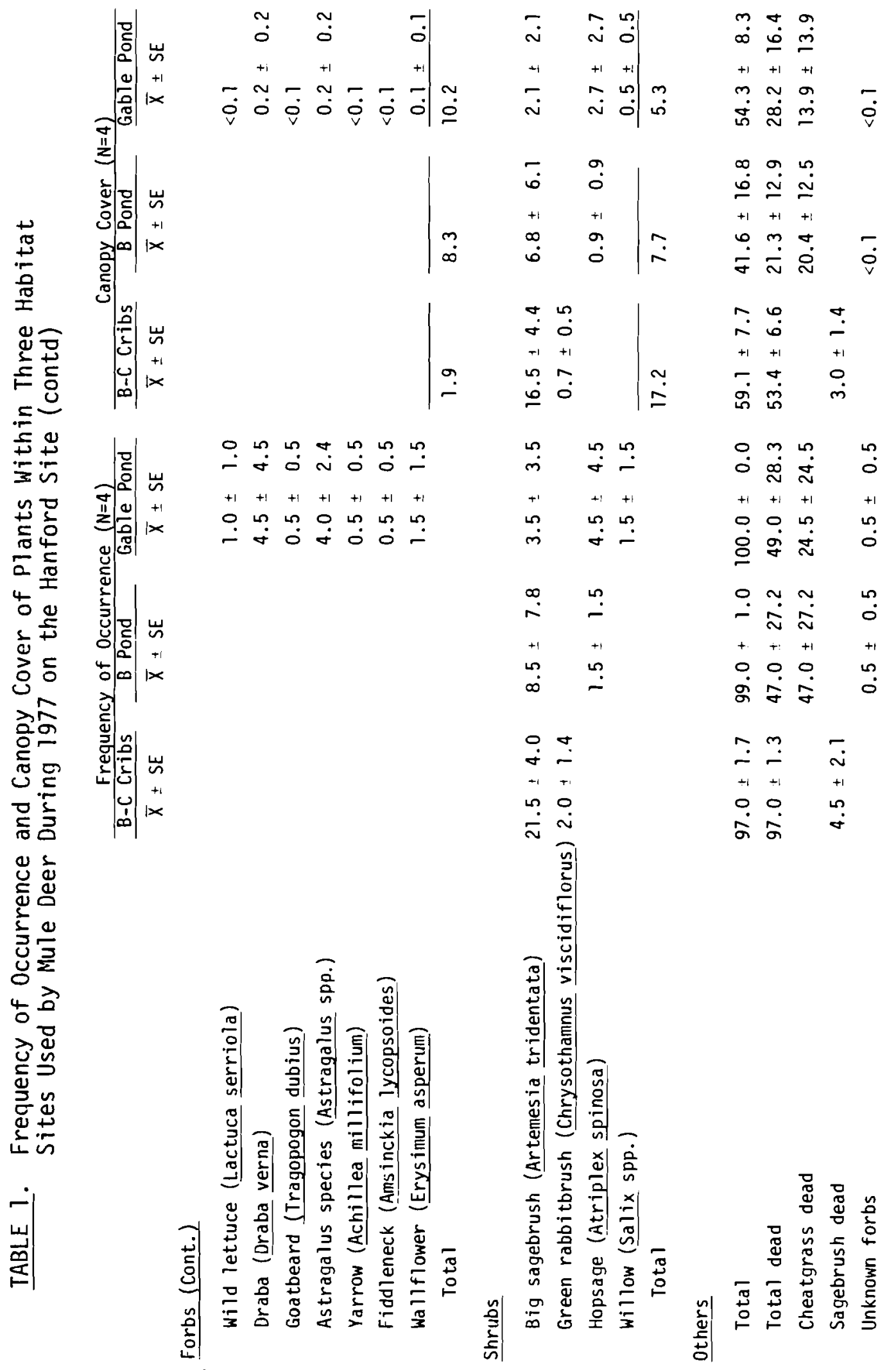


TABLE 2. Botanical Composition of Fecal Pellets Collected from Mule Deer During 1976 from Three Habitat Sites on the Hanford Site

Grasses and Grass-like Plants

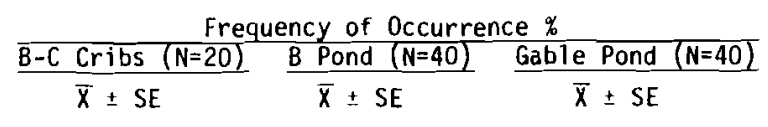

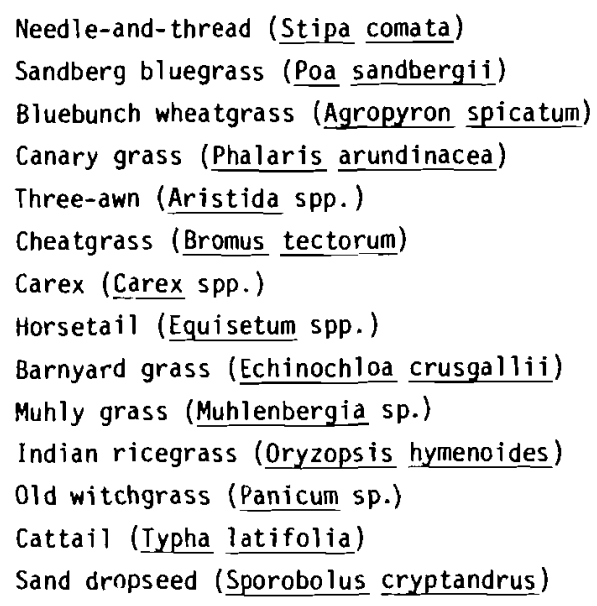

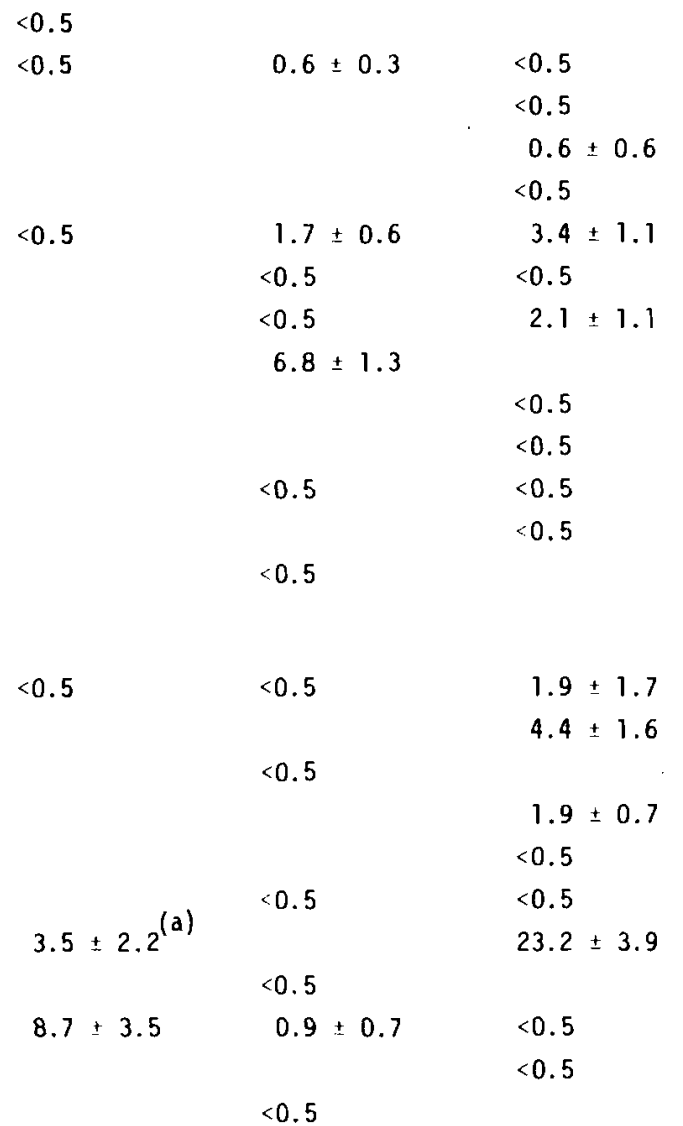

\footnotetext{
(a) These plants are not expected to occur in the B-C Cribs area, which indicates deer would move into this area primarily from the $B$ Pond site.
} 
TABLE 2. Botanical Composition of Fecal Pellets Collected from Mule Deer During 1976 from Three Habitat Sites on the Hanford Site (contd)

\begin{tabular}{|c|c|c|c|}
\hline & $\underline{B-C \operatorname{Cribs}(N=20)}$ & B Pond $(N=40)$ & Gable Pond $(N=40)$ \\
\hline & $\overline{\bar{x}} \pm \mathrm{SE}$ & $\bar{x} \pm$ SE & $\bar{X} \pm S E$ \\
\hline Forbs (Contd) & & & \\
\hline Indian wheat (Plantago purshii) & & & $<0.5$ \\
\hline Willow-weed (Epilobium paniculatum) & & $<0.5$ & $<0.5$ \\
\hline Russian thistle (Salsola kali) & $2.9 \pm 0.7$ & $75.5 \pm 4.0$ & $5.2 \pm 2.2$ \\
\hline Jim Hill mustard (Sisymbrium altissimum) & & & $<0.5$ \\
\hline Goldenrod (Solidago spp.) & $1.4 \pm 0.4^{(\mathrm{a})}$ & $6.1 \pm 1.9$ & $39.7 \pm 5.4$ \\
\hline Globe mallow (Sphaeralcea munroana) & $<0.5$ & $10.9 \pm 4.4$ & $1.8 \pm 1.0$ \\
\hline Buttercup (Ranunculus spp.) & & & $<0.5$ \\
\hline Cormon mullen (Verbascum thapsus) & $<0.5^{(0)}$ & $1.9 \pm 1.9$ & \\
\hline Shrubs & & & \\
\hline Big sagebrush (Artemisia tridentata) & & & $<0.5$ \\
\hline Bitterbrush (Purshia tridentata) & $79.4+7.3$ & & $2.2 \pm 2.2$ \\
\hline Gray Rabbitbrush (Chrysothamnus nauseosus) & & $<0.5$ & $<0.5$ \\
\hline Hopsage (Atriplex spinosa) & & & $<0.5$ \\
\hline Willow (Salix spp.) & $18.1 \pm 5.8^{(\mathrm{a})}$ & $8.3 \pm 2.8$ & $32.3 \div 5.3$ \\
\hline Trees & & & \\
\hline Russian olive (Elaeagnus angustifolia) & & $10.9+4.4$ & \\
\hline thers & & & \\
\hline Moss & & & $<0.5$ \\
\hline Seeds & $1.2 \div 1.2$ & $4.5 \pm 1.6$ & $1.6 \pm 0.6$ \\
\hline Unknown forb & & & $<0.5$ \\
\hline Unknown grass & & $<0.5$ & $<0,5$ \\
\hline Unknown sedge & & $<0.5$ & $<0.5$ \\
\hline
\end{tabular}

(a) These plants are not expected to occur in the B-C Cribs area, which indicates deer would move into this area primarily from $B$ Pond site. 
bitterbrush was the most common species occurring in the diets of deer from the B-C Cribs area, comprising $79 \%$. Russian thistle comprised $76 \%$ and goldenrod $40 \%$ in the diets of deer from B Pond and Gable Mountain Pond, respectively. Forbs provided the mainstay of the mule deer diet and were found more frequently in pellets from Gable Mountain and B Ponds than in pellets from the B-C Cribs site, in which shrubs appeared most often. Shrubs were secondary in importance at the B Pond and Gable Mountain sites, with the grasses and grasslike plants being least important to the mule deer diet. The average dietary composition based upon relative frequency at all sites consisted of $2 \%$ grasses, $43 \%$ shrubs, and $52 \%$ forbs.

Relative preference values are shown in Table 3 . These indices are based on relative frequency values for plants occurring in the diets and in each of the study areas. When a plant species is scarce in the field but is found frequently in the fecal pellets of deer, it is assumed that this particular species of plants were preferred in each study area. Bitterbrush was the preferred species in the B-C Cribs area; both globe mallow and Russian olive were preferred in the B Pond area. Goldenrod was the preferred forage plant in the Gable Mountain Pond area. Cheatgrass was abundant in a 11 areas but was not a preferred food item. However, Russian thistle was abundant in the deer diet and was sought by the deer foraging in the B-C Cribs sites. For additional information see Appendix B.

Similarity indices and rank-order correlation coefficients comparing dietary and habitat analyses of the three areas are presented in Table 4. Similarity indices show the similarity between categories occurring at the study sites, while rank-order correlation coefficients show relationships among the categories when the sites are compared. A strong relationship occurred in diets of deer between the B-C Cribs and Gable Mountain areas. This indicates that the food items were present in different amounts as indicated by similarity indices, but in the same relative proportions as shown by a significant correlation coefficient. Preference indices of forage plants consumed by deer showed low similarity indices when the three sites were compared, indicating that forage plants highly preferred at one site may not be sought at another site.

Similarity indices comparing frequency of occurrence of plants in the habitats among the sites were low, ranging from $23 \%$ to $28 \%$. Significant rankorder correlations did occur when comparisons between B-C Cribs versus Gable Mountain Pond and B Pond versus Gable Mountain Pond were evaluated. This shows that these habitats were related in plant frequency. Canopy cover did not show any high similarity indices; however, a significant correlation did occur between B Pond and Gable Mountain Pond.

RADIONUCLIDE ANALYSIS OF FECAL PELLETS

Gamma-energy analyses were conducted on the 100 groups of deer fecal pellets for $144 \mathrm{Ce}, 106 \mathrm{Ru}, 137 \mathrm{Cs}, 95 \mathrm{Zr}, 54 \mathrm{Mn}, 65 \mathrm{Zn}, 40 \mathrm{~K}$ and $60 \mathrm{Co}$. Pellet groups ranging in age from very recent to very old were included in the analysis. The pellets were collected from the three study areas and a control area in 
TABLE 3. Preference Indices of Mule Deer by Food Items on Three Areas of the Hanford Reservation

\section{B-C Cribs B Pond Gable Mtn. Pond}

Grasses and Grass-1ikes

Needle-and-thread
Sandberg bluegrass
Bluebunch wheatgrass
Canary grass
Three-awn
Cheatgrass
Carex
Horse-tail
Barnyard grass
Muhly grass
Indian ricegrass
Old witchgrass
Cattail
Sand dropseed
rbs

$\begin{array}{rrr}<1 & & \\ <1 & <1 & <1 \\ & & 2 \\ & & 6 \\ <1 & <1 & <1 \\ & & <1 \\ & 2 & 3 \\ & 1 & 2 \\ 68 & <1 \\ & & 1 \\ & <1 & <1 \\ & <1 & <1\end{array}$

Astragalus

Bassia

Tansy mustard

Wild lettuce

Bladderpod

Lupine species

White sweet clover

Microsteris

Evening primrose

Phlox

Prickly pear

Indian wheat

Willow-weed

Russian thistle

Jim Hill mustard

Goldenrod

Globe mallow

Buttercup

Common mullen

Shrubs

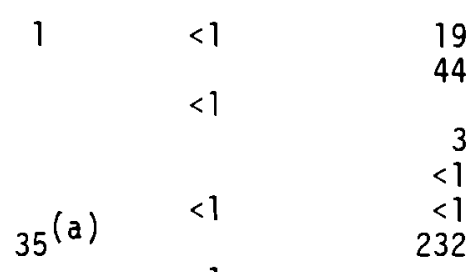

87

$<1$
9

$<1$

6

$<1$

3

2

$<1$

$<1$

$<1$

Big sagebrush

Bitterbrush

Gray rabbit brush

Hopsage

Trees

Willow

$\begin{array}{lrr} & <1 & 3 \\ & 2 & 1 \\ 29 & 3 & <1 \\ 14(a) & 61 & <1 \\ 1 & 109 & 397 \\ { }_{1}(a) & 2 & 18 \\ & & 3\end{array}$

Russian olive

0thers

Moss

Seeds

$9^{(a)}$

45

$<1$

$<1$

$794+12$
$\quad<1$

$\begin{array}{lll}181^{(a)} & 83 & <1\end{array}$

109

(a) These plants are not expected to occur in the B-C Cribs area, which indicates jeer would move into this area primarily from B Pond site. 
TABLE 4. Average Similarity Indices (\%) and Rank-Order Correlation Coefficients $\left(r_{s}\right)$ of Mule Deer Diets, Preference Indices, Frequency of 0ccurrence and Canopy Cover When the Three Areas are Compared.

\begin{tabular}{|c|c|c|c|c|c|c|c|c|c|}
\hline \multirow[b]{2}{*}{ Category } & \multicolumn{3}{|c|}{ B-C Cribs Versus B Pond } & \multicolumn{3}{|c|}{ B-C Cribs Versus Gable Pond } & \multicolumn{3}{|c|}{ B Pond Versus Gable Pond } \\
\hline & $\mathrm{n}$ & $x \pm S E$ & $r_{s}$ & $n$ & $\bar{x} \pm S E$ & $r_{s}$ & $n$ & $\bar{x} \pm S E$ & $r_{s}$ \\
\hline Deer Diets & $\overline{27}$ & $\overline{10 \pm 4}$ & $\overline{+0.28}$ & $\overline{38}$ & $\overline{10 \pm 4}$ & $+0.54^{(a)}$ & $\overline{43}$ & $\overline{16 \pm 4}$ & +0.05 \\
\hline Forage Preference & 27 & $12 \pm 4$ & +0.17 & 38 & $7 \pm 3$ & $+0.40^{(b)}$ & 43 & $19 \pm 5$ & +0.01 \\
\hline Frequency of occurrence & 22 & $26 \pm 8$ & +0.34 & 31 & $23 \pm 6$ & $+0.40^{(b)}$ & 28 & $35 \pm 8$ & $+0.67^{(a)}$ \\
\hline Canopy Cover & 22 & $22 \pm 6$ & +0.18 & 31 & $18 \pm 6$ & +0.26 & 28 & $31 \pm 7$ & $+0.67(a)$ \\
\hline
\end{tabular}

(a) Significant at $\alpha \leq 0.01$

(b) Significant at $a \leq 0.05$

Deer Creek Canyon near Provo, Utah. The pellets used for radiochemical analysis were also used for the dietary analysis. Statistical comparisons followed the Bonferroni T-test statistical method (Miller, 1966). All tests were at the $p=0.008$ level.

Table 5 presents the values for $144 \mathrm{Ce}$ in the deer pellets. The average values are approximately the same for the three study sites with no statistical differences. Maximum values of $111 \mathrm{pCi} / \mathrm{g}$ and $99 \mathrm{pCi} / \mathrm{g}$ were detected in pellet groups collected from B Pond and Gable Mountain Pond study areas, respectively. Counting variability was high as shown by the negative data for the minimum values.

Ruthenium-106 values were higher $(p=0.008)$ in pellets collected from the $B-C$ Cribs and Gable Mountain Pond areas than those in the control (Table 6). The $B$ Pond and the control values were not significantly different statistically. The wide range of values indicates the variability found during the sample analysis.

Cesium-137 values are presented in Table 7 . No differences in ${ }^{137} \mathrm{Cs}$ values were found between the pellets from the control and the B-C Cribs study area. Values for $B$ Pond and Gable Mountain Pond averaged $15 \mathrm{pCi} / \mathrm{g}$ and $16 \mathrm{pCi} / \mathrm{g}$, respectively, and were higher than the control. Maximum $13{ }^{3} \mathrm{Cs}$ values were $100 \mathrm{pCi} / \mathrm{g}$ at $\mathrm{B}$ Pond and $108 \mathrm{pCi} / \mathrm{g}$ at Gable Pond. Variability among these samples was much lower as the maximum and minimum values indicate. Figure 2 presents the distribution of $137 \mathrm{Cs}$ found in the deer fecal pellets. Most of the values are below $38 \mathrm{pCi} / \mathrm{g}$, although five samples are found in the higher range of the spectra.

Table 8 presents values for $95 \mathrm{Zr}$ in the pellets. No real differences are evident among the various locations, and the values appear to reflect worldwide fallout levels. 
TABLE 5. Cerium-144 Content of Mule Deer Feces $(\mathrm{pCi} / \mathrm{g}$ ) Collected from the 200 Area Plateau, and Deer Creek, Utah.

\begin{tabular}{|c|c|c|c|c|}
\hline Location & $\underline{n}$ & $\bar{x} \pm S E$ & Maximum & Minimum \\
\hline B-C Cribs & 20 & $15.4 \pm 6.2$ & 70.8 & $-40.3^{(a)}$ \\
\hline B Pond & 40 & $22.3 \pm 3.8$ & 98.6 & -15.5 \\
\hline Gable Mountain Pond & 40 & $21.7 \pm 6.2$ & 110.6 & -63.1 \\
\hline Control (Utah) & 7 & $13.7 \pm 8.9$ & 46.0 & -16.5 \\
\hline
\end{tabular}

(a) Negative values are less than analytical background.

TABLE 6. Ruthenium-106 Content of Mule Deer Feces ( $\mathrm{pCi} / \mathrm{g}$ ) Collected from the 200 Area Plateau and Deer Creek, Utah.

\begin{tabular}{|c|c|c|c|c|}
\hline Location & $n$ & $X \pm S E$ & Maximum & Minimum \\
\hline B-C Cribs & 20 & $57.3 \pm 5.7$ & 103.7 & 18.3 \\
\hline B Pond & 40 & $5.8 \pm 10.2$ & 134.6 & $-105.0^{(a)}$ \\
\hline Gable Mountain Pond & 40 & $42.8 \pm 6.0$ & 148.7 & -48.2 \\
\hline Control (Utah) & 7 & $-27.7 \pm 9.59$ & 8.2 & -57.7 \\
\hline
\end{tabular}

(a) Negative values are less than analytical background. 
TABLE 7. Cesium-137 Content of Mule Deer Feces ( $\mathrm{pCi/g)} \mathrm{Collected} \mathrm{from}$ the 200 Area Plateau and Deer Creek, Utah.

\begin{tabular}{|c|c|c|c|c|}
\hline Location & $n$ & $\bar{x} \pm S E$ & Maximum & Minimum \\
\hline B-C Cribs & 20 & $1.6 \pm 1.1$ & 17.7 & $-4.4^{(a)}$ \\
\hline B Pond & 40 & $14.8 \pm 2.9$ & 99.6 & -1.5 \\
\hline Gable Mountain Pond & 40 & $16.0 \pm 3.6$ & 127.8 & -3.9 \\
\hline Control (Utah) & 7 & $0.5 \pm 0.9$ & 3.3 & -2.1 \\
\hline
\end{tabular}

(a) Negative values are less than analytical background.

TABLE 8. Zirconium-95 Content of Mule Deer Feces ( $p C i / g$ ) Collected from the 200 Area Plateau and Deer Creek, Utah.

\begin{tabular}{|c|c|c|c|c|}
\hline Location & $n$ & $\bar{x} \pm S E$ & Maximum & Minimum \\
\hline B-C Cribs & 20 & $-1.4 \pm 0.5$ & 2.9 & $-5.2^{(a)}$ \\
\hline B Pond & 40 & $-1.4 \pm 1.1$ & 11.5 & -16.4 \\
\hline Gable Mountain Pond & 40 & $2.1 \pm 0.6$ & 13.2 & -3.5 \\
\hline Control (Utah) & 7 & $-0.3 \pm 3.9$ & 15.1 & -15.5 \\
\hline
\end{tabular}

(a) Negative values are less than analytical background. 


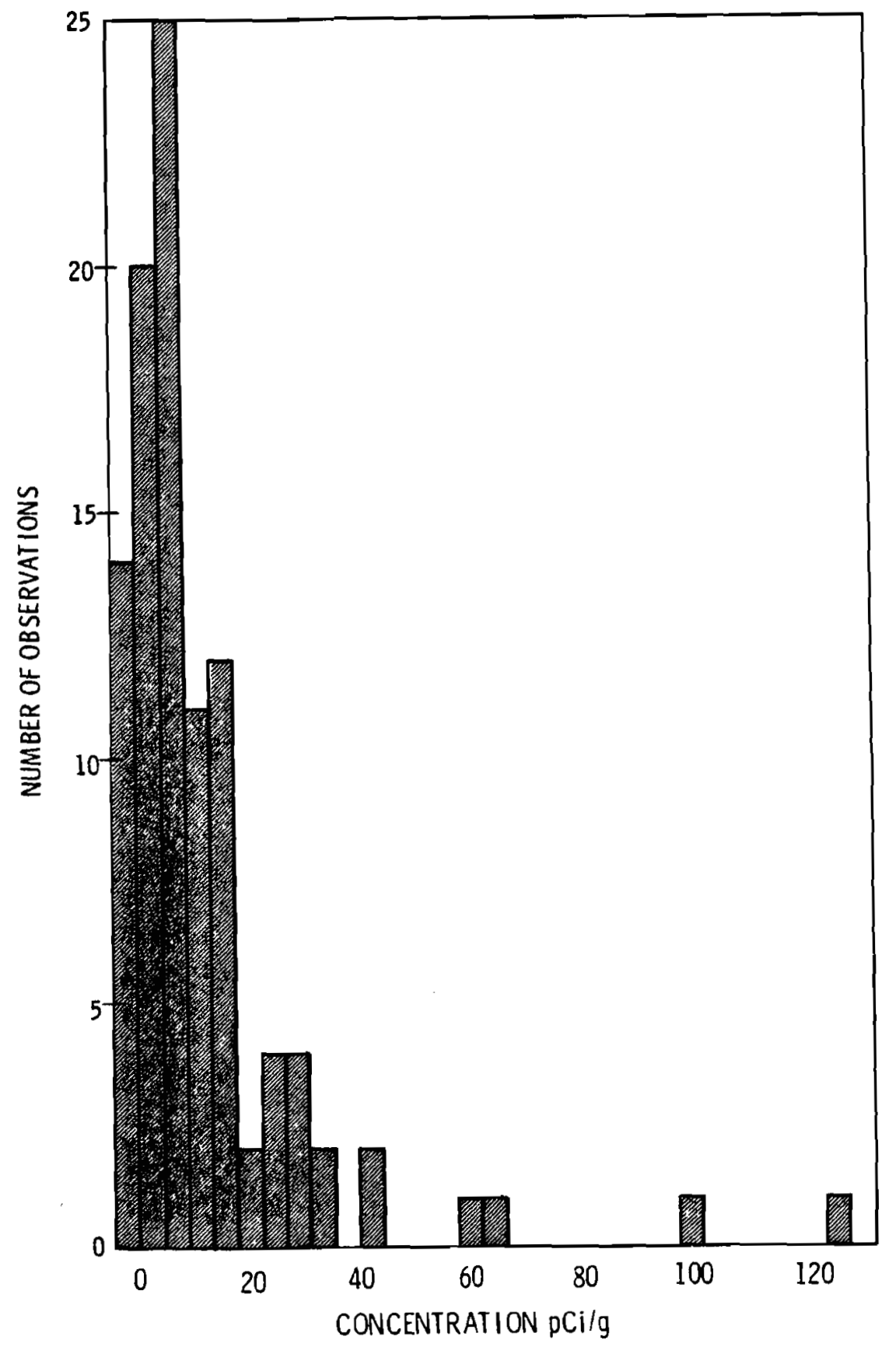

FIGURE 2. Cesium-137 Found in Mule Deer Pellets from B-C Cribs, B Ponds, and Gable Mountain Pond Areas on the Hanford Site 
Manganese-54 in the pellets appears to be at background levels with no differences evident among locations (Table 9). Table 10 presents values for $65 \mathrm{Zn}$ in the pellets. No real differences among locations are evident with the control pellets showing the highest average value of $9 \mathrm{pCi} / \mathrm{g}$.

Values of $40_{K}$, a naturally occurring radionuclide, were highest in pellets collected from $B$ Pond, averaging $45 \mathrm{pCi} / \mathrm{g}$ (Table 11). The other two areas and the control had values at background levels. These differences probably reflect differences in the potassium content of forage and in the food habits of deer within the study areas.

TABLE 9. Manganese-54 Content of Mule Deer Feces ( $\mathrm{pCi} / \mathrm{g}$ ) Collected from the 200 Area Plateau and Deer Creek, Utah.

\begin{tabular}{|c|c|c|c|c|}
\hline Location & $n$ & $\bar{x} \pm S E$ & Maximum & Minimum \\
\hline B-C Cribs & 20 & $-0.1 \pm 0.4$ & 3.2 & $-2.4^{(a)}$ \\
\hline B Pond & 40 & $1.0 \pm 0.3$ & 5.1 & -2.3 \\
\hline Gable Mountain Pond & 40 & $-3.6 \pm 0.5$ & 3.2 & -10.3 \\
\hline Control (Utah) & 7 & $-2.9 \pm 1.1$ & 3.8 & -5.1 \\
\hline
\end{tabular}

(a) Negative values are less than analytical background.

TABLE 10. Zinc-65 Content of Mule Deer Feces ( $\mathrm{pCi} / \mathrm{g}$ ) Collected from the 200 Area Plateau and Deer Creek, Utah.

\begin{tabular}{|c|c|c|c|c|}
\hline Location & $n$ & $\bar{x} \pm S E$ & Maximum & Minimum \\
\hline B-C Cribs & 20 & $-8.2 \pm 1.3$ & 5.2 & $-17.1^{(a)}$ \\
\hline B Pond & 40 & $4.6 \pm 1.0$ & 17.3 & -11.7 \\
\hline Gable Mountain Pond & 40 & $1.1 \pm 0.7$ & 8.8 & -7.2 \\
\hline Control (Utah) & 7 & $8.8 \pm 1.8$ & 14.1 & 2.7 \\
\hline
\end{tabular}

(a) Negative values are less than analytical background. 
TABLE 11. Potassium-40 Content of Mule Deer Feces ( $p C i / g$ ) from the 200 Area Plateau and Deer Creek, Utah.

\begin{tabular}{|c|c|c|c|c|}
\hline Location & $\underline{n}$ & $\bar{x} \pm S E$ & Maximum & Minimum \\
\hline B-C Cribs & 20 & $-10.1 \pm 5.8$ & 37.1 & $-64.0^{(a)}$ \\
\hline B Pond & 40 & $45.3 \pm 5.3$ & 97.4 & -21.5 \\
\hline Gable Mountain Pond & 40 & $-29.2 \pm 5.3$ & 34.3 & -126.6 \\
\hline Control (Utah) & 7 & $-10.2 \pm 11.0$ & 29.2 & -47.3 \\
\hline
\end{tabular}

(a) Negative values are less than analytical background.

Cobalt-average values did not exceed background levels at any of the three study areas or control (Table 12). Strontium-12 analys is was conducted on the 12 fecal pellet groups collected at the B Pond and Gable Mountain sites which showed the highest $137 \mathrm{Cs}$ values among the total pellet groups sampled. Strontium-90 content in the pellet groups from the Gable Mountain area ranged from 2 to $184 \mathrm{pCi} / \mathrm{g}$ (Table 13). Pellet groups from B Pond ranged from 12 to $107 \mathrm{pCi} / \mathrm{g}$.

Dietary analysis of the two pellet groups from the Gable Mountain Pond area which showed the highest $90 \mathrm{Sr}$ values $(20$ and $184 \mathrm{pCi} / \mathrm{g})$ revealed the following plant composition based on relative percentage values: willow (59\%), white sweet clover $(33 \%)$, goldenrod $(7 \%)$, and garex $(1 \%)$. Plants found in the five pellet groups collected from B Pond from ${ }^{90} \mathrm{Sr}$ analysis were: Russian thistle $(70 \%)$, goldenrod $(7 \%)$, barnyard grass $(6 \%)$, globe mallow $(5 \%)$, willow $(4 \%)$, cheatgrass $(3 \%)$, seeds $(3 \%)$, and others $(2 \%)$.

These analyses indicate that the plants located adjacent to the pond areas represent a potential mechanism for $90 \mathrm{Sr}$ transport. So 11 was not considered as a mechanism in these analyses since the soil particles were not detectable in the microscopic analysis of the diets. Additional information on $90 \mathrm{Sr}$ content of plants adjacent to these areas is necessary for assessing the future need for vegetation control measures. 
TABLE 12. Cobalt-60 Content of Mule Deer Feces ( $\mathrm{pCi} / \mathrm{g}$ )

Collected from the 200 Area Plateau and

Deer Creek, Utah.

\begin{tabular}{|c|c|c|c|c|}
\hline Location & n & $\bar{x} \pm S E$ & Maximum & Minimum \\
\hline B-C Cribs & 20 & $-0.2 \pm 0.5$ & 3.2 & $-4.4^{(a)}$ \\
\hline B Pond & 40 & $-0.7 \pm 0.3$ & 3.2 & -6.6 \\
\hline Gable Mountain Pond & 40 & $-0.6 \pm 0.4$ & 6.3 & -6.6 \\
\hline Control (Utah) & 7 & $-2.3 \pm 1.2$ & 1.5 & -7.6 \\
\hline
\end{tabular}

(a) Negative values are less than analytical background.

TABLE 13. Strontium-90 ( $\mathrm{pCi} / \mathrm{g}$ ) Analysis of Deer Pellet Groups Collected Adjacent to B Pond and Gable Mountain Pond on the Hanford Site During 1976

\begin{tabular}{|c|c|c|c|c|}
\hline Location & Class & $\begin{array}{r}\text { Diet } \\
\text { No. (a) }\end{array}$ & $\begin{array}{l}\text { Radiation } \\
\text { No. (a) }\end{array}$ & $\begin{array}{l}\text { Result } \\
(\mathrm{pCi} \mathrm{g})\end{array}$ \\
\hline Gable Mountain Pond & Recent & 49 & 406 & 3.9 \\
\hline Gable Mountain Pond & old & 42 & 410 & 5.1 \\
\hline Gable Mountain Pond & Recent & 56 & 411 & 1.6 \\
\hline Gable Mountain Pond & 01d & 63 & 428 & 4.0 \\
\hline Gable Mountain Pond & Recent & 79 & 434 & 6.1 \\
\hline Gable Mountain Pond & Recent & 73 & 436 & 20.4 \\
\hline Gable Mountain Pond & Recent & 76 & 437 & 184.3 \\
\hline B Pond & Recent & 08 & 466 & 130.1 \\
\hline B Pond & old & 05 & 468 & 12.0 \\
\hline B Pond & 01d & 02 & 470 & 63.6 \\
\hline B Pond & Recent & 25 & 488 & 50.7 \\
\hline B Pond & Recent & 24 & 489 & 107.0 \\
\hline
\end{tabular}

(a) Diet and radiation sample numbers are cross-referenced for more detail in Appendices $B$ and $C$, (e.g., Diet analysis of pellet group 49 can be found in Appendix B. Additional radiation analyses for the same pellet group can be found under 406 in Appendix C). 


\section{DISCUSSION}

In the arid region of southcentral Washington at the Hanford Site, mule deer are primarily found along the riparian habitat of the Columbia River (Hedlund, 1975). Deer are commonly found at Hanford near man-made ponds which contain effluent from nuclear waste operations. These ponds and surrounding vegetation provide a source of water, food and cover which are important in supporting wildlife. The Hanford Site supports a sizable population of mule deer that have been protected from hunting since 1943.

The amount of forage available to large herbivores is generally sparse in arid regions. Similarity indices show that the three study areas are not alike if frequency of occurrence and canopy cover of plants are considered. The number of plant taxa varied at each location with those areas near man-made ponds richest in species composition. B Pond and Gable Mountain Pond areas were more closely related in plant frequency and canopy cover than with vegetation in the B-C Cribs area.

Dietary habits of the mule deer in southcentral Washington on Artemisia tridentata/Poa sandbergii associations (Daubenmire, 1970) have not been reported in the literature. Most authors have reported dietary habits of mule deer on bunchgrass rangelands, which have some sagebrush and bitterbrush with understory species of bluebunch wheatgrass (Agropyron spicatum), cheatgrass and Sandberg bluegrass (Edgerton and Smith, 1971; Wi1kins, 1957; Morris and Schwartz, 1957; Ne11is and Ross, 1969; and Lovaas, 1958). These studies have been conducted in areas which receive more precipitation than the Hanford Site.

The mule deer select forbs as the mainstay of their diet at $B$ Pond and Gable Mountain Pond areas. Shrubs were the second most common food item, while grasses were a minor portion of the total diet. At the B-C Cribs habitat, shrubs were the major food item found in the pellets, followed by forbs and grasses. In Montana, Wilkins (1957) found that summer forbs were the most important, that shrubs were of less importance, and that grass was least often eaten. Wilkins also found that bitterbrush was the most important species in the deers' diet during the fall. Edgerton (1971) in eastern Oregon showed that forbs were important in the spring. However, with the decline of spring forbs, the amounts of grasses and shrubs in the diets of deer increased sharply. During the fall, grasses and sedges were important while shrubs became a major constituent of the diets in forested areas.

At Hanford, six forage species provided the mainstay of the mule deer diet from the $B$ Pond habitat, and three forage species made up most of the diet from B-Cribs area and Gable Mountain Pond habitat. Several species of plants were abundant in the habitat but were rarely eaten. These species were apparently unpalatable, although others were not eaten because more preferred species were available. Short et al. (1966) have shown that the normal and predictable variation in plant chemistry caused by seasonal changes in plant phenology and physiology have important implications in the nutrition and physiology of deer. These major forage species consumed by the deer may be related to the nutrient requirement of the animal. 
The food items found in deer pellets from the B-C Cribs and Gable Mountain Pond habitats related more closely than those found at $B$ Pond. Preference indices at the B-C Cribs and Gable Mountain Pond habitats showed the same general trends; the deer at both sites showed a significant relationship in forage preference. Generally these deer were selecting different foods at each site even though some plants were common to all sites. Cheatgrass was abundant to all three habitats, but dietary analysis indicated that it provided only a small part of the total diet. Generally seven plants were highly preferred and sought out in a far greater proportion than their availability within each of the three habitat types. Other forage plants were found in both the diet and habitats either in the same relative frequency or less in the diet than in the habitat, indicating that deer do not especially prefer these plants. The habitats are dissimilar in frequency of occurrence and canopy cover.

\section{RADIONUCLIDES IN FECAL PELLETS OF DEER}

Mule deer at Hanford are wide-ranging, as the tags collected from hunters by check stations indicate (Hedlund, 1975). Deer tags have been returned from hunter kills at Mattawa, 25 miles upstream, and from another 50 miles downstream near the confluence of the Columbia and Walla Walla rivers. The farthest documented distance that a Hanford deer was killed by a hunter was 70 miles away at Soap Lake.

Mule deer occupying areas on the Hanford Site where low-level radionuclide contamination is present, such as retired waste disposal trenches and cribs (0'Farre11 and Gilbert, 1975; Klepper, et a1., 1979) and ponds and streams (Cushing and Watson, 1974; Emery and McShane, 1978), have the potential for transporting radionuclides to surrounding areas. Concentrations of $137 \mathrm{Cs}$ in deer fecal pellets averaged 1.6 (range $0-17.7$ ) $\mathrm{pCi} / \mathrm{g}$ at $\mathrm{B}-\mathrm{C}$ Cribs, 14.8 (range 0-99.6) $\mathrm{pCi} / \mathrm{g}$ at $\mathrm{B}-$ Pond and 16.0 (range $0-127.8$ ) $\mathrm{pCj} / \mathrm{g}$ at Gable Mountain Pond. The average $137 \mathrm{Cs}$ concentration in feces from a control site (Utah) was 0.5 (range $0-3.3$ ) $\mathrm{pCi} / \mathrm{g}$ (Table 7 ).

Strontium-90 analysis of seven fecal pellet samples ranged from 1.6 to $184.3 \mathrm{pCi} / \mathrm{g}$. Five samples from near B-Pond ranged from 12.0 to $130.1 \mathrm{pCi} / \mathrm{g}$ (Table 13). These data indicate that deer are ingesting radionuclides. Forage plants are a likely vehicle for the radioactive materials, but contaminated water is also a possibility. The absence of soil particles in microscope slide preparations used for dietary analysis indicates that direct ingestion of contaminated soil was probably not a major contributor to the radionuclide burden in the feces.

Analyzing deer pellets from many locations provides information on the levels of radioactive contaminants ingested by deer. Trapping programs at sensitive sites for whole body counting could provide reliable data on the same arimal over several years. Monitoring environmental contaminants by analysis of deer fecal pellets is an attractive method since it would not harm the animal. 


\section{REFERENCES}

Baumgartner, L. L. and A. C. Martin. "Plant Histology as an Aid in Squirrel Food-habit Studies." J. Wild1. Manage. 3:266-268. 1939.

Cline, J. F., D. W. Uresk and W. H. Rickard. "Plants and Soil of a Sagebrush Community on the Hanford Reservation." Northwest Sci. 51:60-70. 1977.

Cushing, C. E. and D. G. Watson. "Aquatic Studies of Gable Mountain Pond." BNWL-1884. Batte11e, Pacific Northwest Laboratories, Richland, WA. 1974.

Daubenmire, R. "A Canopy-coverage Method of Vegetation Analysis." Northwest Sci. 33:43-64. 1959.

Daubenmire, R. "Steppe Vegetation of Washington." Wash. Agric. Exp. Stn. Tech. Bu11. 62. 131 pp. 1970.

Edgerton, P. J. and J. G. Smith. "Seasonal Forage Use by Deer and Elk on the Starkey Experimental Forest and Range, Oregon." U.S. Forest Service Research Paper, PNW-112. 12 pp. 1971.

Emery, R. M. and M. C. McShane. "Comparative Ecology of Nuclear Waste Ponds and Streams on the Hanford Site." PNL-2499. Pacific Northwest Laboratory, Richland, WA. 1978.

Giles, R. H. "Wildlife Management Techniques," 3rd Ed., Rev., Wildlife Society, Wildlife Techniques Manual Committee, Washington, DC. 623 pp. 1969.

Hajek, B. F. "Soil Survey - Hanford Project in Benton County, Washington." BNWL-234. Battelle, Pacific Northwest Laboratories, Richland, WA. 1977.

Hedlund, J. D. "Tagging Mule Deer Fawns in South-central Washington, 1969-1974." Northwest Sci. 49:153-157. 1975.

Klepper, B., L. E. Rogers, J. D. Hedlund, R. G. Schreckhise and K. R. Price. "Radiocesium Movement in a Gray Rabbitbrush Community." Proceedings of the Environmental Chemistry and Cycling Processes Symposium, D. C. Adriano and I. L. Brisbin, Jr. (ed), Apri1 28-May 1, August, GA. CONF-760429, Savannah River Ecology Laboratory, Aiken, SC. NTIS, Springfield, VA. 1978.

Lovaas, A. L. "Mule Deer Food Habits and Range Use, Little Belt Mountains, Montana." J. Wildl. Manage. 22:275-283. 1958.

Messner, H. E., D. R. Dietz and C. E. Garrett. "A Modification of the Slanting Deer Fence." J. Range Manage. 26:233-235. 1973.

Miller, R. G., Jr. "Simultaneous Statistical Inference." McGraw-Hill Book Company, New York, NY. 272 pp. 1966.

Morris, M. S. and J. E. Schwartz. "Mule Deer and Elk Food Habits on the National Bison Range." J. Wild1. Manage. 21:189-193. 1957. 
Nellis, C. H. and R. L. Ross. "Changes in Mule Deer Food Habits Associated with Herd Reduction." J. Wildl. Manage. 33:191-195. 1969.

0'Farre11, T. P. and R. 0. Gilbert. "Transport of Radioactive Materials by Jackrabbits on the Hanford Reservation." Health Physics 29:9-15. 1975.

Oosting, H. J. "The Study of Plant Communities." W. H. Freeman and Company, San Francisco, CA. 440 pp. 1956.

Short, H. L., D. R. Dietz and E. E. Remmenga. "Selected Nutrients in Mule Deer Browse Plants." Ecology 4:222-229. 1966.

Siege1, S. "Non-parametric Statistics for the Behavioral Sciences." McGrawHil1, NY. 312 pp. 1956.

Snedecor, G. W. and W. G. Cochran. "Statistical Methods." Iowa State Univ. Press, Ames. 593 pp. 1967.

Sparks, D. R. and J. C. Malechek. "Estimating Percentage Dry Weight in Diets Using Microscopic Technique." J. Range Mange. 21:264-265. 1968.

Wilkins, B. T. "Range Use, Food Habits, and Agricultural Relationships of Mule Deer, Bridger Mountains, Montana. J. Wild1. Manage. 21:159-169. 1957. 
APPENDIX A

SUMMARIES OF CANOPY COVER AND OCCURRENCE FREQUENCY

BY PLANT SPECIES

FROM B-C CRIBS, B POIND AND GABLE MOUNTAIN POND HABITATS 
Listing of Plant Species and Code Names from

B-C Cribs, B Pond and Gable Mountain Areas

Grasses and Grass-like Plants

Cheatgrass (Bromus tectorum)

Sandberg bluegrass (Poa sandbergii)

Sixweek fescue (Festuca octoflora)

Three stem bullrush (Scirpus americanus)

Big stem bullrush (Scripus spp.)

Little stem bullrush (Scirpus spp.)

\begin{tabular}{l} 
Code \\
\hline Brte, Brte D (Dead) \\
Pose, Posa \\
Feoc \\
Scam, BBR \\
Scsp, BBR \\
Scsp, LBR
\end{tabular}

Forbs

Pale comandra (Comandra pallida)

Buckwheat (Eriogonum spp.)

Tansy mustard (Descurania pinnata)

Ragweed (Ambrosia acanthicarpa)

Sl imleaf qoosefoot (Chenopodium leptophyllum)

Wing-nut crypthantha (Cryptantha pterocarya)

Russian thistle (Salsola kali)

Jim hill mustard (Sisymbrium altissimum)

Matted cryptantha (Cryptantha circumscissa)

Threadleaf phacelia (Phacelia Tinearis)

Common mullen (Verbascum thapsus)

Wild lettuce (Lactuca serriola)

Draba (Draba verna)

Goatbeard (Tragopogon dubius)

Astragalus (Astragalus spp.)

Yarrow (Achil1ea mil1ifolium)

Wa 11 flower (Erysimum asperum)

Copa

Ersp, Erni

Depi

Amac, Amps

Chle

Crpt.

Saka

Sial

Crci

Phli, Hydr

Veth, Mul1

Lase

Drve

Trdu

Astr.

Acmi

Fiddleneck (Amsinckia Tycopsoides)

Unknown

Eras

Amly

Unk1. Unkf

Shrubs

Big sagebrush (Artemesia tridentata)

Green rabbitbrush (Chrysothamuus viscidiflorus)

Hopsage (Atriplex spinosa)

Willow (Salix spp.)

Artr, Artr D (Dead)

Chvi

Atsp., Grsp.

Sasp, Salx.

Others

TOTAL

TOTAL dead

TOTAL

$N(0), N(1), N(2)$

$B C-1, B C-2, \ldots$

$B C=B-C$ Cribs Area

$B P=B$ Pond Area

GMP $=$ Gable Mountain Pond Area

Totaled total of all species

Totaled total of all dead species

Total sum of above

Diversity indicies

These indicate line transects 
Summary of Canopy Cover (percent Cover by Species) at BC-Cribs

\begin{tabular}{|c|c|c|c|c|c|c|}
\hline & $B C_{-1}(a)$ & $B C-2^{(a)}$ & $B C-3^{(a)}$ & $B C-4^{(a)}$ & MEAN & STAN ERR \\
\hline TOTL & 48.750 & 64.500 & 78.350 & 44.750 & 59.088 & 7.707 \\
\hline $5 L M X$ & 2437.5 & 3225.5 & 3917.5 & 2237.5 & 2954.4 & 385.3 \\
\hline $5 x * x$ & 186431.3 & $27+462.5$ & 340918.8 & 154056.3 & 238967.2 & 42449.9 \\
\hline SD & 37.144 & 36.826 & 26.335 & 33.175 & 33.370 & 2.512 \\
\hline$C V$ & .762 & .571 & .336 & .741 & .603 & .099 \\
\hline SE & 5.253 & 5.208 & 3.724 & 4.692 & 4.719 & .355 \\
\hline $95 \% \cup$ & 58.783 & 74.447 & 85.463 & 53.711 & 68.101 & 8.385 \\
\hline $95^{\circ} \dot{L} \mathrm{~L}$ & 38.717 & 54.553 & 71.237 & 35.789 & 50.074 & 7.028 \\
\hline$D E A D$ & 42.100 & 64.550 & 65.050 & 41.850 & 53.388 & 6.590 \\
\hline SLMX & 2105.0 & 3227.5 & 3252.5 & 2092.5 & 2669.4 & 329.5 \\
\hline $5 x * x$ & 147912.5 & 274468.8 & 255706.3 & 140868.8 & 204739.1 & 35081.5 \\
\hline SD & 34.786 & 36.738 & 30.011 & 32.980 & 33.629 & 1.429 \\
\hline $\mathrm{CV}$ & .826 & .569 & .461 & .788 & .661 & .087 \\
\hline SE & 4.919 & 5.196 & 4.244 & 4.664 & 4.756 & .202 \\
\hline $95 \%$ & 51.496 & 74.473 & 73.156 & 50.758 & 62.471 & 6.976 \\
\hline $95^{\circ} \circ \mathrm{L}$ & 32.704 & 54.627 & 56.944 & 32.942 & 44.304 & 6.204 \\
\hline BRTE & 2.200 & 1.700 & 4.400 & 1.300 & 2.400 & .692 \\
\hline SLMX & 110.0 & 85.0 & 220.0 & 65.0 & 120.0 & 34.6 \\
\hline $5 x * x$ & 1212.5 & 212.5 & 2987.5 & 162.5 & 1143.8 & 660.4 \\
\hline$S D$ & 4.450 & 1.178 & 6.420 & 1.262 & 3.327 & 1.282 \\
\hline $\mathrm{CV}$ & 2.023 & .693 & 1.459 & .971 & 1.286 & .292 \\
\hline SE & .629 & .167 & .908 & .178 & .471 & .181 \\
\hline $\left.95^{\prime \prime}\right)^{\prime}$ & 3.402 & 2.018 & 6.134 & 1.641 & 3.299 & 1.038 \\
\hline $95^{\circ} \mathrm{L}$ & .998 & 1.382 & 2.566 & .959 & 1.501 & .345 \\
\hline ARTR & 14.900 & 13.250 & 29.000 & 8.850 & 16.500 & 4.358 \\
\hline $\operatorname{SLM} X$ & 745.0 & 662.5 & 1450.0 & 422.5 & 825.0 & 217.9 \\
\hline $5 x=x$ & 64075.0 & 55656.3 & 140312.5 & 35456.3 & 73875.0 & 22945.4 \\
\hline SD & 32.880 & 30.931 & 44.781 & 25.371 & 33.491 & 4.086 \\
\hline$C V$ & 2.207 & 2.334 & 1.544 & 2.867 & 2.238 & .272 \\
\hline SE & 4.650 & 4.374 & 6.333 & 3.588 & 4.736 & .578 \\
\hline $95^{\prime \prime}: L$ & 23.781 & 21.605 & 41.096 & 15.703 & 25.546 & 5.462 \\
\hline $95 \%$ L & 6.019 & 4.895 & 16.904 & 1.997 & 7.454 & 3.254 \\
\hline POSE & .950 & 8.100 & 4.100 & .750 & 3.475 & 1.722 \\
\hline $\operatorname{sLM} x$ & 47.5 & 405.0 & 205.0 & 37.5 & 173.8 & 86.1 \\
\hline $5 x^{*} x$ & 681.3 & 38062.5 & 5387.5 & 468.8 & 11150.0 & 9042.4 \\
\hline SD & 3.603 & 26.643 & 9.633 & 2.999 & 10.719 & 5.515 \\
\hline $\mathrm{CV}$ & 3.793 & 3.289 & 2.350 & 3.998 & 3.357 & .368 \\
\hline SE & .510 & 3.768 & 1.362 & .424 & 1.516 & .780 \\
\hline $95 \%$ U & 1.923 & 15.297 & 6.702 & 1.560 & 6.370 & 3.212 \\
\hline $95^{\circ}: \mathrm{L}$ & -.023 & .903 & 1.498 & -.060 & .580 & .232 \\
\hline $\mathrm{CRCl}$ & 1.000 & .150 & .100 & .250 & .375 & .211 \\
\hline SL:MX & 50.0 & 7.5 & 5.0 & 12.5 & 18.8 & 10.5 \\
\hline $5 x^{*} x$ & 500.0 & 18.8 & 12.5 & 31.3 & 140.6 & 119.9 \\
\hline SD & 3.030 & .600 & .495 & .758 & $1.2 \geq 1$ & .606 \\
\hline $\mathrm{Cr}$ & 3.030 & 3.998 & 4.949 & 3.030 & $3 .-52$ & .460 \\
\hline SE & .429 & .085 & .070 & .107 & .173 & .086 \\
\hline $95^{\circ} \circ \mathrm{L}$ & 1.819 & .312 & .234 & .455 & .705 & .374 \\
\hline $95^{\circ} \% \mathrm{~L}$ & .181 & -.012 & -.034 & .045 & .045 & .047 \\
\hline COPA & .350 & 0.000 & 0.000 & 1.250 & .400 & .295 \\
\hline $5 L 1 X$ & 17.5 & 0.0 & 0.0 & 62.5 & 20.0 & 14.8 \\
\hline $5 x * x$ & 231.3 & 0.0 & 0.0 & 906.2 & 284.4 & 214.3 \\
\hline $\mathrm{SD}$ & 2.143 & 0.000 & 0.000 & 4.111 & 1.564 & .988 \\
\hline $\mathrm{CV}$ & 6.124 & 0.000 & 0.000 & 3.289 & 2.353 & $1.4 \pi$ \\
\hline SE & .303 & 0.000 & 0.000 & .581 & .221 & .740 \\
\hline $95 \circ \mathrm{L}$ & .929 & 0.000 & 0.000 & 2.360 & .822 & .562 \\
\hline
\end{tabular}

(a) $B C-1$ indicates $B C$-Cribs transect $1, B C-2$ indicates $B C$-Cribs transect 2 , etc. 


\section{Summary of Canopy Cover (Percent Cover by Species) at BC-Cribs (contd.)}

\begin{tabular}{|c|c|c|c|c|c|c|}
\hline $95 \% \mathrm{~L}$ & -.229 & 0.000 & 0.000 & .140 & -.022 & .028 \\
\hline ERNI & .100 & 0.000 & 0.000 & .100 & .050 & .029 \\
\hline $\operatorname{sen} x$ & 5.0 & 0.0 & 0.0 & 5.0 & 2.5 & 1.4 \\
\hline$S X^{*} X$ & 12.5 & 0.0 & 0.0 & 12.5 & 6.3 & 3.6 \\
\hline SD & .495 & 0.000 & 0.000 & .495 & .247 & .143 \\
\hline $\mathrm{CV}$ & 4.949 & 0.000 & 0.000 & 4.949 & 2.474 & 1.429 \\
\hline SE & .070 & 0.000 & 0.000 & .070 & .035 & .020 \\
\hline $95 \% \cup$ & .234 & 0.000 & 0.000 & .234 & .117 & .067 \\
\hline $95 \% \mathrm{~L}$ & -.034 & 0.000 & 0.000 & -.034 & -.017 & -.010 \\
\hline FEOC & .200 & 0.000 & .150 & .300 & .163 & .063 \\
\hline $\operatorname{sum} x$ & 10.0 & 0.0 & 7.5 & 15.0 & 8.1 & 3.1 \\
\hline$S X^{*} x$ & 25.0 & 0.0 & 18.8 & 37.5 & 20.3 & 7.8 \\
\hline SD & .685 & 0.000 & .600 & .821 & .526 & .181 \\
\hline$C V$ & 3.426 & 0.000 & 3.998 & 2.736 & 2.540 & .885 \\
\hline SE & .097 & 0.000 & .085 & .116 & .074 & .026 \\
\hline $95 \% \cup$ & .385 & 0.000 & .312 & .522 & .305 & .171 \\
\hline $95 \% \mathrm{~L}$ & .015 & 0.000 & -.012 & .078 & .020 & .014 \\
\hline DEPI & .750 & .250 & .300 & .300 & .400 & .117 \\
\hline $\operatorname{sen} x$ & 37.5 & 12.5 & 15.0 & 15.0 & 20.0 & 5.9 \\
\hline$S x^{*} x$ & 468.8 & 31.3 & 37.5 & 37.5 & 143.8 & 108.3 \\
\hline SD & 2.999 & .758 & .821 & .821 & 1.349 & .550 \\
\hline $\mathrm{CV}$ & 3.998 & 3.030 & 2.736 & 2.736 & 3.125 & .299 \\
\hline SE & .424 & .107 & .116 & .116 & .191 & .078 \\
\hline $95 \% \cup$ & 1.560 & .455 & .522 & .522 & .764 & .266 \\
\hline $95 \% \mathrm{~L}$ & -.060 & .045 & .078 & .078 & .036 & -.031 \\
\hline ARTRD & 1.950 & 3.750 & 6.400 & 0.000 & 3.025 & 1.361 \\
\hline $\operatorname{SUM} X$ & 97.5 & 187.5 & 320.0 & 0.0 & 151.3 & 68.0 \\
\hline$S X^{*} X$ & 9506.3 & 12543.8 & 26825.0 & 0.0 & 12218.8 & 5553.6 \\
\hline SD & 13.789 & 15.545 & 22.487 & 0.000 & 12.955 & 4.709 \\
\hline $\mathrm{CV}$ & 7.071 & 4.145 & 3.514 & 0.000 & 3.682 & 7.452 \\
\hline SE & 1.950 & 2.198 & 3.180 & 0.000 & 1.832 & .666 \\
\hline $95 \% \cup$ & 5.675 & 7.949 & 12.474 & 0.000 & 6.524 & 2.633 \\
\hline $95 \% \mathrm{~L}$ & -1.775 & -.449 & .326 & 0.000 & -.474 & .089 \\
\hline AMPS & .350 & 0.000 & 0.000 & 2.050 & .600 & .490 \\
\hline $\operatorname{sun} x$ & 17.5 & 0.0 & 0.0 & 102.5 & 30.0 & 24.5 \\
\hline$S x^{*} x$ & 237.3 & 0.0 & 0.0 & 9518.7 & 2437.5 & 2361.0 \\
\hline SD & 2.143 & 0.000 & 0.000 & 13.783 & 3.982 & 3.306 \\
\hline $\mathrm{CV}$ & 6.124 & 0.000 & 0.000 & 6.723 & 3.212 & 1.858 \\
\hline SE & .303 & 0.000 & 0.000 & 1.949 & .563 & .468 \\
\hline $95 \% U$ & .929 & 0.000 & 0.000 & 5.773 & 1.675 & 1.383 \\
\hline $95 \% \mathrm{~L}$ & -.229 & 0.000 & 0.000 & -1.673 & -.475 & -.403 \\
\hline CHLE & .050 & 0.000 & .300 & .100 & .113 & .066 \\
\hline $\operatorname{sum} x$ & 2.5 & 0.0 & 15.0 & 5.0 & 5.6 & 3.3 \\
\hline$S x^{*} x$ & 6.3 & 0.0 & 225.0 & 12.5 & 60.9 & 54.7 \\
\hline SD & .354 & 0.000 & 2.121 & .495 & .742 & .471 \\
\hline $\mathrm{CV}$ & 7.071 & 0.000 & 7.071 & 4.949 & 4.773 & 1.668 \\
\hline SE & .050 & 0.000 & .300 & .070 & .105 & .067 \\
\hline $95 \% \cup$ & .146 & 0.000 & .873 & .234 & .313 & .193 \\
\hline $95 \% \mathrm{~L}$ & -.046 & 0.000 & -.273 & -.034 & -.088 & -.062 \\
\hline $\mathrm{CHVl}$ & 0.000 & 0.000 & 1.950 & .900 & .713 & .464 \\
\hline $\operatorname{suM} x$ & 0.0 & 0.0 & 97.5 & 45.0 & 35.6 & 23.2 \\
\hline $5 x * x$ & 0.0 & 0.0 & 9506.3 & 675.0 & 2545.3 & 2325.8 \\
\hline SD & 0.000 & 0.000 & 13.789 & 3.598 & 4.347 & 3.260 \\
\hline $\mathrm{CV}$ & 0.000 & 0.000 & 7.071 & 3.998 & 2.767 & 1.716 \\
\hline SE & 0.000 & 0.000 & 1.950 & .509 & .615 & .461 \\
\hline $95 \% U$ & 0.000 & 0.000 & 5.675 & 1.872 & 1.887 & 1.344 \\
\hline $95^{\circ}: \mathrm{L}$ & 0.000 & 0.000 & -1.775 & -.072 & -.462 & -.417 \\
\hline CRPT & 0.000 & 0.000 & 0.000 & .050 & .013 & .013 \\
\hline $\operatorname{sen} x$ & 0.0 & 0.0 & 0.0 & 2.5 & .6 & .6 \\
\hline $5 x=x$ & 0.0 & 0.0 & 0.0 & 6.3 & 1.6 & 1.6 \\
\hline
\end{tabular}




$\begin{array}{lrrrrrr}\text { SD } & 0.000 & 0.000 & 0.000 & .354 & .088 & .088 \\ \text { CV } & 0.000 & 0.000 & 0.000 & 7.071 & 1.768 & 1.768 \\ \text { SE } & 0.000 & 0.000 & 0.000 & .050 & .013 & .013 \\ 95^{\circ} \mathrm{L} & 0.000 & 0.000 & 0.000 & .146 & .036 & .036 \\ 95^{\circ} \mathrm{L} & 0.000 & 0.000 & 0.000 & -.046 & -.011 & -.011 \\ \text { TOTAL } & 113.650 & 156.250 & 190.100 & 102.800 & 140.700 & 20.104 \\ \text { SUM X } & 5682.5 & 7812.5 & 9505.0 & 5140.0 & 7035.0 & 1005.2 \\ \text { SX*X } & 1051568.8 & 1659556.3 & 2127537.5 & 824137.5 & 1415700.0 & 295623.1 \\ \text { SD } & 90.998 & 94.637 & 80.893 & 77.689 & 86.054 & 4.028 \\ \text { CV } & .801 & .606 & .426 & .756 & .647 & .085 \\ \text { SE } & 12.869 & 13.384 & 11.440 & 10.987 & 12.170 & .570\end{array}$

\section{Occurrence Frequency (\%) by Plant Species From BC-Cribs}

$\begin{array}{lrrrrrr} & \text { BC-1 } & \text { BC-2 } & \text { BC-3 } & \text { BC-4 } & \text { MEAN } & \text { STAN ERR } \\ \text { TOTAL } & 94.000 & 94.000 & 100.000 & 100.000 & 97.000 & 1.732 \\ \text { DEAD } & 94.000 & 96.000 & 100.000 & 98.000 & 97.000 & 1.292 \\ \text { BRTE } & 38.000 & 68.000 & 46.000 & 52.000 & 51.000 & 6.351 \\ \text { ARTR } & 26.000 & 18.000 & 30.000 & 12.000 & 27.500 & 4.031 \\ \text { POSE } & 8.000 & 20.000 & 30.000 & 10.000 & 17.000 & 5.065 \\ \text { CRCI } & 20.000 & 6.000 & 4.000 & 10.000 & 10.000 & 3.559 \\ \text { COPA } & 4.000 & 0.000 & 0.000 & 10.000 & 3.500 & 2.363 \\ \text { ERNI } & 4.000 & 0.000 & 0.000 & 4.000 & 2.000 & 1.155 \\ \text { FEOC } & 8.000 & 0.000 & 6.000 & 12.000 & 6.500 & 2.500 \\ \text { DEPI } & 10.000 & 10.000 & 12.000 & 12.000 & 11.000 & .577 \\ \text { ARTRD } & 2.000 & 8.000 & 8.000 & 0.000 & 4.500 & 2.062 \\ \text { AMPS } & 4.000 & 0.000 & 0.000 & 6.000 & 2.500 & 1.500 \\ \text { CHLE } & 2.000 & 0.000 & 2.000 & 4.000 & 2.000 & .816 \\ \text { CHWI } & 0.000 & 0.000 & 2.000 & 6.000 & 2.000 & 1.414 \\ \text { CRPT } & 0.000 & 0.000 & 0.000 & 2.000 & .500 & .500\end{array}$


Summary of Canopy Cover (Percent Cover by Species) at B-Pond

\begin{tabular}{|c|c|c|c|c|c|c|}
\hline & BP-1(a) & BP-2(a) & BP-3(a) & BP-4(a) & MEAN & STAN ERR \\
\hline TOTL & 22.300 & 10.400 & 86.450 & 47.450 & 41.650 & 16.812 \\
\hline SUMX & 1115.0 & 520.0 & 4322.5 & 2372.5 & 2082.5 & 840.6 \\
\hline $5 x * x$ & 32600.0 & 8800.0 & 385693.8 & 184928.8 & 153003.1 & 86821.2 \\
\hline SD & 12.565 & 8.320 & 15.658 & 38.424 & 18.742 & 6.731 \\
\hline $\mathrm{CV}$ & .563 & .800 & .781 & .810 & .589 & .147 \\
\hline SE & 1.777 & 1.177 & 2.214 & 5.434 & 2.650 & .952 \\
\hline $95 \% \mathrm{U}$ & 25.694 & 12.647 & 90.679 & 57.829 & 46.712 & 18.630 \\
\hline $95 \% \mathrm{~L}$ & 18.906 & 8.153 & 82.221 & 37.071 & 36.588 & 14.994 \\
\hline BRTE & 15.550 & 1.450 & 2.550 & 3.350 & 5.725 & 3.298 \\
\hline $\operatorname{SUM} X$ & 777.5 & 72.5 & 127.5 & 167.5 & 286.3 & 164.9 \\
\hline$S X * X$ & 17318.8 & 931.2 & 2381.3 & 2106.3 & 5684.4 & 3890.8 \\
\hline SD & 10.330 & 4.106 & 6.478 & 5.615 & 6.632 & 1.326 \\
\hline $\mathrm{CV}$ & .664 & 2.832 & 2.540 & 1.676 & 1.928 & .488 \\
\hline SE & 1.461 & .581 & .976 & .794 & .938 & .188 \\
\hline $95 \% U$ & 18.340 & 2.559 & 4.300 & 4.867 & 7.516 & 3.656 \\
\hline $95 \% \mathrm{~L}$ & 12.760 & .341 & .800 & 1.833 & 3.934 & 2.940 \\
\hline SAKA & 4.750 & 7.650 & .050 & 0.000 & 3.113 & 1.878 \\
\hline SUM X & 237.5 & 382.5 & 2.5 & 0.0 & 155.6 & 93.9 \\
\hline$S X \cdot X$ & 2468.8 & 4893.8 & 6.3 & 0.0 & 1842.2 & 1171.5 \\
\hline SD & 5.231 & 6.337 & .354 & 0.000 & 2.980 & 1.636 \\
\hline $\mathrm{CV}$ & 1.101 & .828 & 7.071 & 0.000 & 2.250 & 1.624 \\
\hline SE & .740 & .896 & .050 & 0.000 & .421 & .231 \\
\hline $95 \% \cup$ & 6.163 & 9.362 & .146 & 0.000 & 3.918 & 2.320 \\
\hline $95 \% \mathrm{~L}$ & 3.337 & 5.938 & -.046 & 0.000 & 2.307 & 1.436 \\
\hline
\end{tabular}

(a) BP-1 indicates B-Pond transect $1, B P-2$ indicates B-Pond transect 2 , etc. 
Summary of Canopy Cover (Percent Cover by Species) at B-Pond (contd.)

\begin{tabular}{|c|c|c|c|c|c|c|}
\hline SIAL & 5.600 & .250 & .200 & 0.000 & 1.513 & 1.364 \\
\hline $\sin x$ & 280.0 & 12.5 & 10.0 & 0.0 & 75.6 & 68.2 \\
\hline $5 x * x$ & 4450.0 & 31.3 & 25.0 & 0.0 & 1126.6 & 1107.8 \\
\hline SD & 7.669 & .758 & .685 & 0.000 & 2.278 & 1.805 \\
\hline$C V$ & 1.369 & 3.030 & 3.426 & 0.000 & 1.956 & .790 \\
\hline $\mathrm{SE}$ & 1.085 & .107 & .097 & 0.000 & .322 & .255 \\
\hline $95 \% U$ & 7.672 & .455 & .385 & 0.000 & 2.128 & 1.851 \\
\hline $95 \%$ L & 3.528 & .045 & .015 & 0.000 & .897 & .876 \\
\hline ERSP & 1.300 & 3.600 & 0.000 & 0.000 & 1.225 & .849 \\
\hline SUM $X$ & 65.0 & 180.0 & 0.0 & 0.0 & 61.3 & 42.4 \\
\hline$S x=x$ & 725.0 & 3825.0 & 0.0 & 0.0 & 1137.5 & 912.0 \\
\hline SD & 3.615 & 8.052 & 0.000 & 0.000 & 2.917 & 1.912 \\
\hline$C V$ & 2.781 & 2.237 & 0.000 & 0.000 & 1.254 & .733 \\
\hline SE & .511 & 1.139 & 0.000 & 0.000 & .413 & .270 \\
\hline $95 \% U$ & 2.277 & 5.775 & 0.000 & 0.000 & 2.013 & 1.365 \\
\hline $95 \%$ L & .323 & 1.425 & 0.000 & 0.000 & .437 & .332 \\
\hline FEOC & .100 & 0.000 & 0.000 & .200 & .075 & .048 \\
\hline $\operatorname{suM} X$ & 5.0 & 0.0 & 0.0 & 10.0 & 3.8 & 2.4 \\
\hline $5 x * x$ & 12.5 & 0.0 & 0.0 & 25.0 & 9.4 & 6.0 \\
\hline SD & .495 & 0.000 & 0.000 & .685 & .295 & .175 \\
\hline$C V$ & 4.949 & 0.000 & 0.000 & 3.426 & 2.094 & 1.248 \\
\hline SE & .070 & 0.000 & 0.000 & .097 & .042 & .025 \\
\hline $95 \% U$ & .234 & 0.000 & 0.000 & .385 & .155 & .095 \\
\hline $95 \%$ L & -.034 & 0.000 & 0.000 & .015 & -.005 & .001 \\
\hline $\mathrm{CRCl}$ & .050 & 0.600 & 0.000 & .200 & .063 & $.04 i$ \\
\hline $\operatorname{sun} x$ & 2.5 & 0.0 & 0.0 & 10.0 & 3.1 & 2.4 \\
\hline$S x * x$ & 6.3 & 0.0 & 0.0 & 25.0 & 7.8 & 5.9 \\
\hline SD & .354 & 0.000 & 0.000 & .685 & .260 & .164 \\
\hline $\mathrm{Cl}$ & 7.071 & 0.000 & 0.000 & 3.426 & 2.624 & 1.688 \\
\hline SE & .050 & 0.000 & 0.000 & .097 & .037 & .023 \\
\hline $95 \%$ U & .146 & 0.000 & 0.000 & .385 & .133 & .092 \\
\hline $95 \% \mathrm{~L}$ & -.046 & 0.000 & 0.000 & .015 & -.008 & .003 \\
\hline HYDR & 0.000 & .100 & 0.000 & 0.000 & .025 & .025 \\
\hline $\operatorname{SLM} X$ & 0.0 & 5.0 & 0.0 & 0.0 & 1.3 & 1.3 \\
\hline $5 x * x$ & 0.0 & 12.5 & 0.0 & 0.0 & 3.1 & 3.1 \\
\hline SD & 0.000 & .495 & 0.000 & 0.000 & .124 & .124 \\
\hline$C V$ & 0.000 & 4.949 & 0.000 & 0.000 & 1.237 & 1.237 \\
\hline SE & 0.000 & .070 & 0.000 & 0.000 & .017 & .017 \\
\hline $95 \% U$ & 0.000 & .234 & 0.000 & 0.000 & .058 & .058 \\
\hline $95 \% \mathrm{~L}$ & 0.000 & -.034 & 0.000 & 0.000 & -.008 & -.008 \\
\hline UNKL & 0.000 & .050 & 0.000 & 0.000 & .013 & .013 \\
\hline SUMX & 0.0 & 2.5 & 0.0 & 0.0 & .6 & .6 \\
\hline$S x^{*} X$ & 0.0 & 6.3 & 0.0 & 0.0 & 1.6 & 1.6 \\
\hline SD & 0.000 & .354 & 0.000 & 0.000 & .088 & .088 \\
\hline$C V$ & 0.000 & 7.071 & 0.000 & 0.000 & 1.768 & 1.768 \\
\hline SE & 0.000 & .050 & 0.000 & 0.000 & .013 & .013 \\
\hline $95 \% \cup$ & 0.000 & .146 & 0.000 & 0.000 & .036 & .036 \\
\hline $95 \%$ L & 0.000 & -.046 & 0.000 & 0.000 & -.011 & -.011 \\
\hline MULL & 0.000 & .200 & 0.000 & 0.000 & .050 & .050 \\
\hline $\operatorname{SUM} X$ & 0.0 & 10.0 & 0.0 & 0.0 & 2.5 & 2.5 \\
\hline $5 x * x$ & 0.0 & 25.0 & 0.0 & 0.0 & 6.3 & 6.3 \\
\hline SD & 0.000 & .685 & 0.000 & 0.000 & .171 & .171 \\
\hline $\mathrm{CV}$ & 0.000 & 3.426 & 0.000 & 0.000 & .856 & .856 \\
\hline SE & 0.000 & .097 & 0.000 & 0.000 & .024 & .024 \\
\hline $95^{\circ}, U$ & 0.000 & .385 & 0.000 & 0.000 & .096 & .096 \\
\hline $95 \% \mathrm{~L}$ & 0.000 & .015 & 0.000 & 0.000 & .004 & .004 \\
\hline
\end{tabular}


Summary of Canopy Cover (Percent Cover by Species) at B-Pond (contd.)

\begin{tabular}{|c|c|c|c|c|c|c|}
\hline TOTLD & 0.000 & 0.000 & 52.500 & 32.850 & 21.338 & 12.956 \\
\hline $\operatorname{SUM} X$ & 0.0 & 0.0 & 2625.0 & 1642.5 & 1066.9 & 647.8 \\
\hline $5 x * x$ & 0.0 & 0.0 & 188962.5 & 105281.3 & 73560.9 & 45776.7 \\
\hline SD & 0.000 & 0.000 & 32.309 & 32.364 & 16.168 & 9.335 \\
\hline $\mathrm{CV}$ & 0.000 & 0.000 & .615 & .985 & .400 & .243 \\
\hline $\mathrm{SE}$ & 0.000 & 0.000 & 4.569 & 4.577 & 2.287 & 1.320 \\
\hline $95 \% U$ & 0.000 & 0.000 & 61.227 & 41.592 & 25.705 & 15.477 \\
\hline $95 \%$ L & 0.000 & 0.000 & 43.773 & 24.108 & 16.970 & 10.434 \\
\hline BRTED & 0.000 & 0.000 & 51.050 & 30.650 & 20.425 & 12.506 \\
\hline $\operatorname{SUM} X$ & 0.0 & 0.0 & 2552.5 & 1532.5 & 1021.3 & 625.3 \\
\hline$S x * x$ & 0.0 & 0.0 & 187843.8 & 95556.3 & 70850.0 & 45034.6 \\
\hline$S D$ & 0.000 & 0.000 & 34.267 & 31.489 & 16.439 & 9.508 \\
\hline $\mathrm{CV}$ & 0.000 & 0.000 & .671 & 1.027 & .425 & .256 \\
\hline $\mathrm{SE}$ & 0.000 & 0.000 & 4.846 & 4.453 & 2.325 & 1.345 \\
\hline $95 \% \cup$ & 0.000 & 0.000 & 60.306 & 39.156 & 24.865 & 15.074 \\
\hline $95 \% \mathrm{~L}$ & 0.000 & 0.000 & 41.794 & 22.744 & 15.985 & 9.938 \\
\hline POSE & 0.000 & 0.000 & 29.700 & 2.400 & 8.025 & 7.247 \\
\hline SUM X & 0.0 & 0.0 & 1485.0 & 120.0 & 401.3 & 362.4 \\
\hline$S x^{*} X$ & 0.0 & 0.0 & 67425.0 & 5550.0 & 18243.8 & 16445.9 \\
\hline SD & 0.000 & 0.000 & 21.816 & 10.363 & 8.045 & 5.200 \\
\hline $\mathrm{CV}$ & 0.000 & 0.000 & .735 & 4.318 & 1.263 & 1.033 \\
\hline SE & 0.000 & 0.000 & 3.085 & 1.466 & 1.138 & .735 \\
\hline $95 \%$ U & 0.000 & 0.000 & 35.593 & 5.199 & 10.198 & 8.652 \\
\hline $95 \% \mathrm{~L}$ & 0.000 & 0.000 & 23.807 & -.399 & 5.852 & $5.8+3$ \\
\hline ATSP & 0.000 & 0.000 & 3.700 & 0.000 & .925 & .925 \\
\hline SUMX & 0.0 & 0.0 & 185.0 & 0.0 & 46.3 & 46.3 \\
\hline$S x * x$ & 0.0 & 0.0 & 12537.5 & 0.0 & 3134.4 & 3134.4 \\
\hline SD & 0.000 & 0.000 & 15.553 & 0.000 & 3.888 & 3.888 \\
\hline $\mathrm{CV}$ & 0.000 & 0.000 & 4.204 & 0.000 & 1.051 & 1.051 \\
\hline SE & 0.000 & 0.000 & 2.200 & 0.000 & .550 & .550 \\
\hline $95 \% \cup$ & 0.000 & 0.000 & 7.901 & 0.000 & 1.975 & 1.975 \\
\hline $95 \% \mathrm{~L}$ & 0.000 & 0.000 & -.501 & 0.000 & -.125 & -.125 \\
\hline ARTR & 0.000 & 0.000 & 1.950 & 25.200 & 6.788 & 6.155 \\
\hline SUM X & 0.0 & 0.0 & 97.5 & 1260.0 & 339.4 & 307.7 \\
\hline$S x \cdot x$ & 0.0 & 0.0 & 9506.3 & 105412.5 & 28729.7 & 25659.0 \\
\hline SD & 0.000 & 0.000 & 13.789 & 38.772 & 13.140 & 9.141 \\
\hline$C V$ & 0.000 & 0.000 & 7.071 & 1.539 & 2.152 & $\cdot 1.679$ \\
\hline SE & 0.000 & 0.000 & 1.950 & 5.483 & 1.858 & 1.293 \\
\hline $95 \% \cup$ & 0.000 & 0.000 & 5.675 & 35.673 & 10.337 & 8.624 \\
\hline $95 \% \mathrm{~L}$ & 0.000 & 0.000 & -1.775 & 14.727 & 3.238 & 3.686 \\
\hline DEP! & 0.000 & 0.000 & .050 & .300 & .088 & .072 \\
\hline SUMX & 0.0 & 0.0 & 2.5 & 15.0 & 4.4 & 3.6 \\
\hline$S x=x$ & 0.0 & 0.0 & 6.3 & 37.5 & 10.9 & 9.0 \\
\hline SD & 0.000 & 0.000 & .354 & .821 & .294 & .194 \\
\hline $\mathrm{CV}$ & 0.000 & 0.000 & 7.071 & 2.736 & 2.452 & 1.669 \\
\hline SE & 0.000 & 0.000 & .050 & .116 & .042 & .028 \\
\hline $95 \%$ U & 0.000 & 0.000 & .146 & .522 & .167 & .124 \\
\hline $95 \% \mathrm{~L}$ & 0.000 & 0.000 & -.046 & .078 & .008 & .019 \\
\hline TOTAL & 49.650 & 23.700 & 228.200 & 142.600 & 111.038 & 46.654 \\
\hline $\operatorname{SUM} X$ & 2482.5 & 1185.0 & 11410.0 & 7130.0 & 5551.9 & 2332.7 \\
\hline $5 x * x$ & 152631.3 & 45912.5 & 2816887.5 & 1700575.0 & 1179001.6 & 664080.6 \\
\hline SD & 24.485 & 19.075 & 65.951 & 118.135 & 56.911 & 22.937 \\
\hline$C V$ & .493 & .805 & .289 & .828 & .604 & .130 \\
\hline SE & 3.463 & 2.698 & 9.327 & 16.707 & 8.048 & 3.244 \\
\hline $95 \%$ U & 56.264 & 28.852 & 246.014 & 174.510 & 126.410 & 52.850 \\
\hline $95 \% \mathrm{~L}$ & 43.036 & 18.548 & 210.386 & 110.690 & 95.665 & 40.459 \\
\hline
\end{tabular}


Relative Cover and Species Diversity of M.O. Hill in Ecology (1973)

$\begin{array}{lrrrrrr} & \mathbf{B P}-1 & \mathbf{B P}-\mathbf{2} & \mathbf{B P}-\mathbf{3} & \mathbf{B P}-\mathbf{4} & \text { MEAN } & \text { STAN ERR } \\ \text { TOTL } & .449 & .439 & .379 & .333 & .400 & .027 \\ \text { BRTE } & .313 & .061 & .011 & .023 & .102 & .071 \\ \text { SAKA } & .096 & .323 & .000 & 0.000 & .105 & .076 \\ \text { SIAL } & .113 & .011 & .001 & 0.000 & .031 & .027 \\ \text { ERSP } & .026 & .152 & 0.000 & 0.000 & .045 & .036 \\ \text { FEOC } & .002 & 0.000 & 0.000 & .001 & .001 & .001 \\ \text { CRCI } & .001 & 0.000 & 0.000 & .001 & .001 & .000 \\ \text { HYDR } & 0.000 & .004 & 0.000 & 0.000 & .001 & .001 \\ \text { UNKL } & 0.000 & .002 & 0.000 & 0.000 & .001 & .001 \\ \text { MULL } & 0.000 & .008 & 0.000 & 0.000 & .002 & .002 \\ \text { TOTLD } & 0.000 & 0.000 & .230 & .230 & .115 & .066 \\ \text { BRTED } & 0.000 & 0.000 & .224 & .215 & .110 & .063 \\ \text { POSE } & 0.000 & 0.000 & .130 & .017 & .037 & .031 \\ \text { ATSP } & 0.000 & 0.000 & .016 & 0.000 & .004 & .004 \\ \text { ARTR } & 0.000 & 0.000 & .009 & .177 & .046 & .044 \\ \text { DEPI } & 0.000 & 0.000 & .000 & .002 & .001 & .001 \\ \text { TOTAL } & 49.650 & 23.700 & 228.200 & 142.600 & 111.038 & 46.654 \\ \text { N(0) } & 7.000 & 8.000 & 10.000 & 9.000 & 8.500 & .645 \\ \text { N(1) } & 3.701 & 3.699 & 4.366 & 4.615 & 4.095 & .234 \\ \text { N(2) } & 3.102 & 3.089 & 3.789 & 4.131 & 3.528 & .259\end{array}$

Occurrence Frequency (\%) by Plant Species From B-Pond

$\begin{array}{lrrrrrr} & \text { BP-1 } & \text { BP-2 } & \text { BP-3 } & \text { BP-4 } & \text { MEAN } & \text { STAN ERR } \\ \text { TOTL } & 100.000 & 100.000 & 100.000 & 96.000 & 99.000 & 1.000 \\ \text { BRTE } & 96.000 & 18.000 & 34.000 & 44.000 & 48.000 & 16.872 \\ \text { SAKA } & 90.000 & 96.000 & 2.000 & 0.000 & 47.000 & 26.589 \\ \text { SIAL } & 66.000 & 10.000 & 8.000 & 0.000 & 21.000 & 15.155 \\ \text { ERSP } & 22.000 & 48.000 & 0.000 & 0.000 & 17.500 & 11.413 \\ \text { FEOC } & 4.000 & 0.000 & 0.000 & 8.000 & 3.000 & 1.915 \\ \text { CRCI } & 2.000 & 0.000 & 0.000 & 8.000 & 2.500 & 1.893 \\ \text { HYDR } & 0.000 & 4.000 & 0.000 & 0.000 & 1.000 & 1.000 \\ \text { UNKL } & 0.000 & 2.000 & 0.000 & 0.000 & .500 & .500 \\ \text { MULL } & 0.000 & 8.000 & 0.000 & 0.000 & 2.000 & 2.000 \\ \text { TOTLD } & 0.000 & 0.000 & 98.000 & 90.000 & 47.000 & 27.185 \\ \text { BRTED } & 0.000 & 0.000 & 100.000 & 88.000 & 47.000 & 27.246 \\ \text { POSE } & 0.000 & 0.000 & 90.000 & 10.000 & 25.000 & 21.794 \\ \text { ATSP } & 0.000 & 0.000 & 6.000 & 0.000 & 1.500 & 1.500 \\ \text { ARTR } & 0.000 & 0.000 & 2.000 & 32.000 & 8.500 & 7.848 \\ \text { DEPI } & 0.000 & 0.000 & 2.000 & 12.000 & 3.500 & 2.872\end{array}$


Summary of Canopy Cover (Percent Cover by Species) at Gable Mtn. Pond

\begin{tabular}{|c|c|c|c|c|c|c|}
\hline & GP-1(a) & GP-2(a) & GP-3(a) & GP-4(a) & MEAN & STAN ERR \\
\hline TOTL & 42.400 & 41.450 & 77.100 & 56.350 & 54.325 & 8.321 \\
\hline SLM $x$ & 2120.0 & 2072.5 & 3855.0 & 2817.5 & 2716.3 & 416.0 \\
\hline $5 x * x$ & 128825.0 & 108231.3 & 314737.5 & 218543.8 & 192584.4 & 47237.0 \\
\hline SD & 28.189 & 21.346 & 18.907 & 34.928 & 25.843 & 3.610 \\
\hline$C V$ & .665 & .515 & .245 & .620 & .511 & .094 \\
\hline SE & 3.987 & 3.019 & 2.674 & 4.940 & 3.655 & .511 \\
\hline $95 \% \mathrm{U}$ & 50.014 & 47.216 & 82.207 & 65.785 & 61.305 & 9.296 \\
\hline $95 \% \mathrm{~L}$ & 34.786 & 35.684 & 71.993 & 46.915 & 47.345 & 7.345 \\
\hline BRTE & 38.650 & 6.100 & .050 & 4.600 & 12.350 & 8.861 \\
\hline $\operatorname{sun} x$ & 1932.5 & 305.0 & 2.5 & 230.0 & 617.5 & 443.0 \\
\hline $5 x^{*} x$ & 122918.8 & 6012.5 & 6.3 & 8900.0 & 34459.4 & 29544.6 \\
\hline SD & 31.373 & 9.205 & .354 & 12.651 & 13.395 & 6.528 \\
\hline$C V$ & .812 & 1.509 & 7.071 & 2.750 & 3.035 & 1.404 \\
\hline SE & 4.437 & 1.302 & .050 & 1.789 & 1.894 & .923 \\
\hline $95 \%$ U & 47.124 & 8.586 & .146 & 8.017 & 15.968 & 10.624 \\
\hline $95 \% \mathrm{~L}$ & 30.176 & 3.614 & -.046 & 1.183 & 8.732 & 7.097 \\
\hline $\mathrm{CRCl}$ & .650 & 28.900 & 0.000 & .350 & 7.475 & 7.143 \\
\hline $\operatorname{sun} x$ & 32.5 & 1445.0 & 0.0 & 17.5 & 373.8 & 357.1 \\
\hline $5 x * x$ & 456.3 & 55062.5 & 0.0 & 43.8 & 13890.6 & 13724.3 \\
\hline SD & 2.980 & 16.476 & 0.000 & .876 & 5.083 & 3.849 \\
\hline $\mathrm{CV}$ & 4.585 & .570 & 0.000 & 2.504 & 1.915 & 1.039 \\
\hline SE & .427 & 2.330 & 0.000 & .124 & .719 & .544 \\
\hline $95 \% U$ & 1.455 & 33.350 & 0.000 & .587 & 8.848 & 8.183 \\
\hline $95 \% \mathrm{~L}$ & -.155 & 24.450 & 0.000 & .113 & 6.102 & 6.103 \\
\hline SAKA & 1.850 & 0.000 & 0.000 & 1.050 & .725 & .449 \\
\hline $\operatorname{suM} x$ & 92.5 & 0.0 & 0.0 & 52.5 & 36.3 & 22.5 \\
\hline $5 x * X$ & 981.3 & 0.0 & 0.0 & 506.3 & 371.9 & 235.6 \\
\hline SD & 4.066 & 0.000 & 0.000 & 3.034 & 1.775 & 1.046 \\
\hline $\mathrm{CV}$ & 2.198 & 0.000 & 0.000 & 2.890 & $1.2 \% 2$ & .748 \\
\hline $\mathrm{SE}$ & .575 & 0.000 & 0.000 & .429 & .251 & .748 \\
\hline $95 \% \mathrm{U}$ & 2.948 & 0.000 & 0.000 & 1.870 & 1.204 & .732 \\
\hline $95 \%$ L & .752 & 0.000 & 0.000 & .230 & .246 & .167 \\
\hline SIAL & 2.200 & 0.000 & 0.000 & .550 & .688 & .521 \\
\hline $\operatorname{suM} x$ & 110.0 & 0.0 & 0.0 & 27.5 & 34.4 & 26.0 \\
\hline$S X=x$ & 1400.0 & 0.0 & 0.0 & 256.3 & 414.1 & 334.1 \\
\hline SD & 4.861 & 0.000 & 0.000 & 2.218 & 1.770 & 1.156 \\
\hline$C V$ & 2.210 & 0.000 & 0.000 & 4.033 & 1.561 & .975 \\
\hline SE & .687 & 0.000 & 0.000 & .314 & .250 & .163 \\
\hline $95 \%$ U & 3.513 & 0.000 & 0.000 & 1.149 & 1.166 & .833 \\
\hline $95 \%$ L & .887 & 0.000 & 0.000 & -.049 & .209 & .208 \\
\hline 3BR & 0.000 & .440 & 0.000 & 0.000 & .100 & .100 \\
\hline $\operatorname{suM} x$ & 0.0 & 20.0 & 0.0 & 0.0 & 5.0 & 5.0 \\
\hline $5 x+x$ & 0.0 & 237.5 & 0.0 & 0.0 & 59.4 & 59.4 \\
\hline SD & 0.000 & 2.164 & 0.000 & 0.000 & .541 & .541 \\
\hline$C V$ & 0.000 & 5.410 & 0.000 & 0.000 & 1.353 & 1.353 \\
\hline SE & 0.000 & .306 & 0.000 & 0.000 & .077 & .077 \\
\hline $95 \% \mathrm{U}$ & 0.000 & .985 & 0.000 & 0.000 & .246 & .246 \\
\hline $95 \% \mathrm{~L}$ & 0.000 & -185 & 0.000 & 0.000 & -.046 & -.046 \\
\hline
\end{tabular}

(a) GP-1 indicates Gable Mtn. Pond transect 1, GP-2 indicates Gabie Mtn. Pond transect 2, etc. 
Summary of Canopy Cover (Percent Cover by Species) at Gable Mtn. Pond (contd.)

\begin{tabular}{|c|c|c|c|c|c|c|}
\hline ERSP & 0.000 & 1.650 & 0.000 & 0.000 & .413 & .413 \\
\hline SUMX & 0.0 & 82.5 & 0.0 & 0.0 & 20.6 & 20.6 \\
\hline $5 x * x$ & 0.0 & 956.3 & 0.0 & 0.0 & 239.1 & 239.1 \\
\hline SD & 0.000 & 4.091 & 0.000 & 0.000 & 1.023 & 1.023 \\
\hline $\mathrm{CV}$ & 0.000 & 2.479 & 0.000 & 0.000 & .620 & .620 \\
\hline SE & 0.000 & .579 & 0.000 & 0.000 & .145 & .145 \\
\hline $95 \% U$ & 0.000 & 2.755 & 0.000 & 0.000 & .689 & .689 \\
\hline $95 \% \mathrm{~L}$ & 0.000 & .545 & 0.000 & 0.000 & .136 & .136 \\
\hline LASE & 0.000 & .100 & 0.000 & 0.000 & .025 & .025 \\
\hline $\operatorname{SUM} X$ & 0.0 & 5.0 & 0.0 & 0.0 & 1.3 & 1.3 \\
\hline $5 x * x$ & 0.0 & 12.5 & 0.0 & 0.0 & 3.1 & 3.1 \\
\hline SD & 0.000 & .495 & 0.000 & 0.000 & .124 & .124 \\
\hline $\mathrm{CV}$ & 0.000 & 4.949 & 0.000 & 0.000 & 1.237 & 1.237 \\
\hline SE & 0.000 & .070 & 0.000 & 0.000 & .017 & .017 \\
\hline $95 \%$ U & 0.000 & .234 & 0.000 & 0.000 & .058 & .058 \\
\hline $95 \% \mathrm{~L}$ & 0.000 & -.034 & 0.000 & 0.000 & -.008 & -.008 \\
\hline UNK2 & 0.000 & .050 & 0.000 & 0.000 & .013 & .013 \\
\hline SUMX & 0.0 & 2.5 & 0.0 & 0.0 & .6 & .6 \\
\hline $5 x * x$ & 0.0 & 6.3 & 0.0 & 0.0 & 1.6 & 1.6 \\
\hline SD & 0.000 & .354 & 0.000 & 0.000 & .088 & .088 \\
\hline$C V$ & 0.000 & 7.071 & 0.000 & 0.000 & 1.768 & 1.768 \\
\hline SE & 0.000 & .050 & 0.000 & 0.000 & .013 & .013 \\
\hline $95 \% U$ & 0.000 & .146 & 0.000 & 0.000 & .036 & .036 \\
\hline $95 \%$ L & 0.000 & -.046 & 0.000 & 0.000 & -.011 & -.011 \\
\hline DRVE & 0.000 & .950 & $0.00 \overline{0}$ & 0.000 & .238 & .238 \\
\hline $\operatorname{SUM} X$ & 0.0 & 47.5 & 0.0 & 0.0 & 11.9 & 11.9 \\
\hline$s x * x$ & 0.0 & 493.8 & 0.0 & 0.0 & 123.4 & 123.4 \\
\hline SD & 0.000 & 3.026 & 0.000 & 0.000 & .756 & .756 \\
\hline$C V$ & 0.000 & 3.185 & 0.000 & 0.000 & .796 & .796 \\
\hline SE & 0.000 & .428 & 0.000 & 0.000 & .107 & .107 \\
\hline $95 i u$ & 0.000 & 1.767 & 0.000 & 0.000 & .442 & .442 \\
\hline $95 \div \mathrm{L}$ & 0.000 & .133 & 0.000 & 0.000 & .033 & .033 \\
\hline FEOC & 0.000 & .100 & 0.000 & .300 & .100 & .071 \\
\hline $\operatorname{sun} x$ & 0.0 & 5.0 & 0.0 & 15.0 & 5.0 & 3.5 \\
\hline$S x * x$ & 0.0 & 12.5 & 0.0 & 37.5 & 12.5 & 8.8 \\
\hline SD & 0.000 & .495 & 0.000 & .821 & .329 & .201 \\
\hline $\mathrm{CV}$ & 0.000 & 4.949 & 0.000 & 2.736 & 1.921 & 1.198 \\
\hline SE & 0.000 & .070 & 0.000 & .116 & .047 & .028 \\
\hline $95 \% U$ & 0.000 & .234 & 0.0100 & .522 & .189 & .125 \\
\hline $95 \% \mathrm{~L}$ & 0.000 & -.034 & 0.000 & .078 & .011 & .016 \\
\hline TRDU & 0.000 & .300 & 0.000 & 0.000 & .075 & .075 \\
\hline $\operatorname{SUM} X$ & 0.0 & 15.0 & 0.0 & 0.0 & 3.8 & 3.8 \\
\hline $5 x * x$ & 0.0 & 225.0 & 0.0 & 0.0 & 56.3 & 56.3 \\
\hline SD & 0.000 & 2.121 & 0.000 & 0.000 & .530 & .530 \\
\hline $\mathrm{CV}$ & 0.000 & 7.071 & 0.500 & 0.000 & 1.768 & 1.768 \\
\hline SE & 0.000 & .300 & 0.000 & 0.000 & .075 & .075 \\
\hline $95 \%$ U & 0.000 & .873 & 0.000 & 0.000 & .218 & .218 \\
\hline $95 \% \mathrm{~L}$ & 0.000 & -.273 & 0.000 & 0.000 & -.068 & -.068 \\
\hline BBR & 0.000 & 2.400 & 0.000 & 0.000 & .600 & .600 \\
\hline $\operatorname{SUMX}$ & 0.0 & 120.0 & 0.0 & 0.0 & 30.0 & 30.0 \\
\hline$S x * x$ & 0.0 & 3487.5 & 0.0 & 0.0 & 871.9 & 871.9 \\
\hline SD & 0.000 & 8.081 & 0.000 & 0.000 & 2.020 & 2.020 \\
\hline $\mathrm{CV}$ & 0.000 & 3.367 & 0.000 & 0.000 & .842 & .842 \\
\hline SE & 0.000 & 1.143 & 0.000 & 0.000 & .286 & .286 \\
\hline $95^{\circ} \mathrm{L}$ & 0.000 & 4.583 & 0.0000 & 0.000 & $i .146$ & 1.146 \\
\hline $95 \% \mathrm{~L}$ & 0.000 & .217 & (1) & 0.0100 & $.05 t$ & .054 \\
\hline
\end{tabular}


Summary of Canopy Cover (Percent Cover by Species) at Gable Mtn. Pond (contd.)

\begin{tabular}{|c|c|c|c|c|c|c|}
\hline SALX & 0.000 & 1.800 & 0.000 & 0.000 & .450 & 450 \\
\hline $\operatorname{SUM} X$ & 0.0 & 90.0 & 0.0 & 0.0 & 22.5 & 22.5 \\
\hline$S x * x$ & 0.0 & 3037.5 & 0.0 & 0.0 & 759.4 & 759.4 \\
\hline SD & 0.000 & 7.661 & 0.000 & 0.000 & 1.915 & 1.915 \\
\hline$C V$ & 0.000 & 4.256 & 0.000 & 0.000 & 1.064 & $i .064$ \\
\hline $\mathrm{SE}$ & 0.000 & 1.083 & 0.000 & 0.000 & .271 & .271 \\
\hline $95 \% \mathrm{U}$ & 0.000 & 3.869 & 0.000 & 0.000 & .967 & .967 \\
\hline $95 \% \mathrm{~L}$ & 0.000 & -.269 & 0.000 & 0.000 & -.067 & -.067 \\
\hline LBR & 0.000 & .400 & 0.000 & 0.000 & .100 & .100 \\
\hline SUMX & 0.0 & 20.0 & 0.0 & 0.0 & 5.0 & 5.0 \\
\hline$S x * x$ & 0.0 & 237.5 & 0.0 & 0.0 & 59.4 & $5 y+$ \\
\hline SD & 0.000 & 2.164 & 0.000 & 0.000 & .541 & $5+1$ \\
\hline$C V$ & 0.000 & 5.410 & 0.000 & 0.000 & 1.353 & 1.353 \\
\hline SE & 0.000 & .306 & 0.000 & 0.000 & .077 & .077 \\
\hline $95 \% U$ & 0.000 & .985 & 0.000 & 0.000 & .246 & .246 \\
\hline $\begin{array}{l}95 \% \text { L } \\
\text { ASTR }\end{array}$ & $\begin{array}{l}0.000 \\
0.000\end{array}$ & $\frac{-.785}{.650}$ & $\frac{0.000}{0.000}$ & $\begin{array}{c}0.000 \\
.250\end{array}$ & $\begin{array}{l}-.046 \\
.225\end{array}$ & $\begin{array}{c}-.046 \\
.153\end{array}$ \\
\hline SUMX & 0.0 & 32.5 & 0.0 & 12.5 & 11.3 & 7.7 \\
\hline$S X^{*} X$ & 0.0 & 456.3 & 0.0 & 31.3 & 121.9 & 111.7 \\
\hline SD & 0.000 & 2.980 & 0.000 & .758 & .934 & .705 \\
\hline $\mathrm{Cr}$ & 0.000 & 4.585 & 0.000 & 3.030 & 1.504 & 1.144 \\
\hline$S E$ & 0.000 & +31 & 0.000 & $.3 n^{-}$ & .132 & .100 \\
\hline $95 \% \mathrm{U}$ & 0.000 & 1.455 & 0.000 & .455 & .477 & .344 \\
\hline $95 \% \mathrm{~L}$ & 0.000 & -.155 & 0.000 & .045 & -.027 & -.037 \\
\hline$A C M I$ & 0.000 & .050 & 0.000 & 0.000 & .013 & .013 \\
\hline $\operatorname{suM} x$ & 0.0 & 2.5 & 0.0 & 0.0 & .6 & .6 \\
\hline$S x^{*} x$ & 0.0 & 6.3 & 0.0 & 0.0 & 1.6 & 1.6 \\
\hline SD & 0.000 & .354 & 0.000 & 0.000 & .088 & .088 \\
\hline$C V$ & 0.000 & 7.071 & 0.000 & 0.000 & 1.768 & 1.768 \\
\hline SE & 0.000 & .050 & 0.000 & 0.000 & .013 & .013 \\
\hline $95 \% U$ & 0.000 & .146 & 0.000 & 0.000 & .036 & .036 \\
\hline $95 \% \mathrm{~L}$ & 0.000 & -.046 & 0.000 & 0.000 & -.011 & -.011 \\
\hline TOTLD & 0.000 & 0.000 & 61.300 & 51.650 & 28.238 & 16.421 \\
\hline SUM X & 0.0 & 0.0 & 3065.0 & 2582.5 & 1411.9 & 821.1 \\
\hline $5 x * x$ & 0.0 & 0.0 & 237875.0 & 199693.8 & 109392.2 & 63636.7 \\
\hline SD & 0.000 & 0.000 & 31.941 & 36.786 & 17.182 & 9.969 \\
\hline $\mathrm{CV}$ & 0.000 & 0.000 & .521 & .712 & .308 & .182 \\
\hline SE & 0.000 & 0.000 & 4.517 & 5.202 & 2.430 & 1.410 \\
\hline $95 \%$ U & 0.000 & 0.000 & 69.928 & 61.586 & 32.879 & 19.114 \\
\hline $95 \%$ L & 0.000 & 0.000 & 52.672 & 41.714 & 23.596 & 13.729 \\
\hline POSE & 0.000 & 0.000 & 13.600 & .300 & 3.475 & 3.376 \\
\hline $\operatorname{suMx}$ & 0.0 & 0.0 & 680.0 & 15.0 & 173.8 & 168.8 \\
\hline $5 x * x$ & 0.0 & 0.0 & 17637.5 & 225.0 & 4465.6 & 4390.9 \\
\hline SD & 0.000 & 0.000 & 13.085 & 2.121 & 3.802 & 3.135 \\
\hline$C V$ & 0.000 & 0.000 & .962 & 7.071 & 2.008 & 1.703 \\
\hline $\mathrm{SE}$ & 0.000 & 0.000 & 1.850 & .300 & .538 & .443 \\
\hline $95 \% U$ & 0.000 & 0.000 & 17.134 & .873 & 4.502 & 4.222 \\
\hline $95 \% 1$ & 0.000 & 0.000 & 10.066 & -.273 & 2.448 & 2.529 \\
\hline BRTED & 0.000 & 0.000 & 55.600 & 0.000 & 13.900 & 13.900 \\
\hline SUMX & 0.0 & 0.0 & 2780.0 & 0.0 & 695.0 & 695.0 \\
\hline$S x^{*} X$ & 0.0 & 0.0 & 219162.5 & 0.0 & 54790.6 & 54790.6 \\
\hline SD & 0.000 & 0.000 & 36.308 & 0.000 & 9.077 & 9.077 \\
\hline$C V$ & 0.000 & 0.000 & .653 & 0.000 & .163 & .163 \\
\hline SE & 0.000 & 0.000 & 5.135 & 0.000 & 1.284 & 1.284 \\
\hline $95 \%$ U & 0.000 & 0.000 & 65.407 & 0.000 & 16.352 & 16.352 \\
\hline $95 \% L$ & 0.000 & 0.000 & 45.793 & 0.000 & 11.448 & 11.448 \\
\hline
\end{tabular}


Summary of Canopy Cover (Percent Cover by Species) at Gable Mtn. Pond (contd.)

\begin{tabular}{|c|c|c|c|c|c|c|}
\hline ATSP & 0.000 & 0.000 & 10.850 & 0.000 & 2.713 & 2.713 \\
\hline $\operatorname{SUM} x$ & 0.0 & 0.0 & 542.5 & 0.0 & 135.6 & 135.6 \\
\hline $5 x * x$ & 0.0 & 0.0 & 41593.8 & 0.0 & 10398.4 & 10398.4 \\
\hline SD & 0.000 & 0.000 & 26.995 & 0.000 & 6.749 & 6.749 \\
\hline$C V$ & 0.000 & 0.000 & 2.488 & 0.000 & .622 & .622 \\
\hline SE & 0.000 & 0.000 & 3.818 & 0.000 & .954 & .954 \\
\hline $95 \%$ U & 0.000 & 0.000 & 18.142 & 0.000 & 4.535 & 4.535 \\
\hline $95 \%$ L & 0.000 & 0.000 & 3.558 & 0.000 & .890 & .890 \\
\hline DEPI & 0.000 & 0.000 & 0.000 & 1.000 & .250 & .250 \\
\hline $\operatorname{SuM} x$ & 0.0 & 0.0 & 0.0 & 50.0 & 12.5 & 12.5 \\
\hline$S X^{*} \mathrm{X}$ & 0.0 & 0.0 & 0.0 & 312.5 & 78.1 & 78.1 \\
\hline SD & 0.000 & 0.000 & 0.000 & 2.315 & .579 & .579 \\
\hline$C V$ & 0.000 & 0.000 & 0.000 & 2.315 & .579 & .579 \\
\hline SE & 0.000 & 0.000 & 0.000 & .327 & .082 & .082 \\
\hline $95 \% \cup$ & 0.000 & 0.000 & 0.000 & 1.625 & .406 & .406 \\
\hline $\begin{array}{l}95 \% \text { L } \\
\text { AMAC }\end{array}$ & $\begin{array}{l}0.000 \\
0.000\end{array}$ & $\begin{array}{c}0.000 \\
0.000\end{array}$ & $\begin{array}{r}0.000 \\
0.000\end{array}$ & $\begin{array}{r}.375 \\
.550\end{array}$ & $\begin{array}{l}.094 \\
.138\end{array}$ & $\begin{array}{l}.094 \\
.138\end{array}$ \\
\hline SUMX & 0.0 & 0.0 & 0.0 & 27.5 & 6.9 & 6.9 \\
\hline $5 x * x$ & 0.0 & 0.0 & 0.0 & 68.8 & 17.2 & 17.2 \\
\hline SD & 0.000 & 0.000 & 0.000 & 1.046 & .262 & .262 \\
\hline $\mathrm{CV}$ & 0.000 & 0.000 & 0.000 & 1.902 & .476 & .476 \\
\hline SE & 0.000 & 0.000 & 0.000 & .148 & .037 & .037 \\
\hline $95 \% U$ & 0.000 & 0.000 & 0.000 & .833 & .208 & .208 \\
\hline $95 \% \mathrm{~L}$ & 0.000 & 0.000 & 0.000 & .267 & .067 & .067 \\
\hline AMLY & 0.000 & 0.000 & 0.000 & .050 & .013 & .013 \\
\hline $\operatorname{SuMX}$ & 0.0 & 0.0 & 0.0 & 2.5 & .6 & .6 \\
\hline $5 x * x$ & 0.0 & 0.0 & 0.0 & 6.3 & 1.5 & 1.6 \\
\hline SD & 0.000 & 0.000 & 0.000 & .354 & .088 & .088 \\
\hline$C V$ & 0.000 & 0.000 & 0.000 & 7.071 & 1.768 & 1.768 \\
\hline SE & 0.000 & 0.000 & 0.000 & .050 & .013 & .013 \\
\hline $95 \%$ U & 0.000 & 0.000 & 0.000 & .146 & .036 & .036 \\
\hline $95 \% \mathrm{~L}$ & 0.000 & 0.000 & 0.000 & -.046 & -.011 & -.011 \\
\hline ERAS & 0.000 & 0.000 & 0.000 & .400 & .100 & .100 \\
\hline $\operatorname{SUMX}$ & 0.0 & 0.0 & 0.0 & 20.0 & 5.0 & 5.0 \\
\hline$S x * x$ & 0.0 & 0.0 & 0.0 & 237.5 & 59.4 & 59.4 \\
\hline SD & 0.000 & 0.000 & 0.000 & 2.164 & .541 & .541 \\
\hline$C V$ & 0.000 & 0.000 & 0.000 & 5.410 & 1.353 & 1.353 \\
\hline SE & 0.000 & 0.000 & 0.000 & .306 & .077 & .077 \\
\hline $95 \%$ U & 0.000 & 0.000 & 0.000 & .985 & .246 & .246 \\
\hline $95 \% \mathrm{~L}$ & 0.000 & 0.000 & 0.000 & -.185 & -.046 & -.046 \\
\hline ARTR & 0.000 & 0.000 & 0.000 & 8.650 & 2.163 & 2.163 \\
\hline $\operatorname{sun} x$ & 0.0 & 0.0 & 0.0 & 432.5 & 108.1 & 108.1 \\
\hline $5 x * x$ & 0.0 & 0.0 & 0.0 & 33181.3 & 8295.3 & 8295.3 \\
\hline SD & 0.000 & 0.000 & 0.000 & 24.512 & 6.128 & 6.128 \\
\hline$C V$ & 0.000 & 0.000 & 0.000 & 2.834 & .708 & .708 \\
\hline SE & 0.000 & 0.000 & 0.000 & 3.466 & .867 & .867 \\
\hline $95 \% U$ & 0.000 & 0.000 & 0.000 & 15.271 & 3.818 & 3.818 \\
\hline $95 \% \mathrm{~L}$ & 0.000 & 0.0000 & 0.000 & 2.029 & .507 & .507 \\
\hline TOTAL & 85.750 & 85.300 & 218.500 & 126.050 & 128.900 & $31.35 \bar{i}$ \\
\hline $\sin x$ & 4287.5 & $\$ 265.0$ & 10925.0 & 6302.5 & 6445.0 & $156-.9$ \\
\hline $5 x * x$ & 525318.8 & $4465 \div 5.0$ & $278598-.5$ & 1129306.3 & 1221796.9 & 543238.6 \\
\hline SD & 56.724 & 41.100 & 90.224 & 82.669 & 67.679 & 11.400 \\
\hline$C V$ & .662 & .482 & .413 & .656 & .553 & .063 \\
\hline SE & 8.022 & 5.872 & 12.760 & 11.691 & 9.571 & 1.612 \\
\hline $95 \%$ U & 101.072 & 96.402 & 242.871 & 148.380 & 147.181 & 34.436 \\
\hline $95 \% \mathrm{~L}$ & 70.428 & 74.198 & 194.129 & 103.720 & $1 ` i 0.619$ & 28.279 \\
\hline
\end{tabular}


Occurrence Frequency (\%) by Plant Species From Gable Mtn. Pond

$\begin{array}{lrrrrrr} & \text { GP-1 } & \text { GP-2 } & \text { GP-3 } & \text { GP-4 } & \text { MEAN } & \text { STAN ERR } \\ \text { TOTL } & 100.000 & 100.000 & 100.000 & 100.000 & 100.000 & 0.000 \\ \text { BRTE } & 88.000 & 48.000 & 2.000 & 48.000 & 46500 & 17.576 \\ \text { CRCI } & 6.000 & 100.000 & 0.000 & 14.000 & 30.000 & 23.509 \\ \text { SAKA } & 34.000 & 0.000 & 0.000 & 22.000 & 14.000 & 8.446 \\ \text { SIAL } & 28.000 & 0.000 & 0.000 & 12.000 & 10.000 & 6.633 \\ \text { 3BR } & 0.000 & 6.000 & 0.000 & 0.000 & 1.500 & 1.500 \\ \text { ERSP } & 0.000 & 26.000 & 0.000 & 0.000 & 6.500 & 6.500 \\ \text { LASE } & 0.000 & 4.000 & 0.000 & 0.000 & 1.000 & 1.000 \\ \text { LNK2 } & 0.000 & 2.000 & 0.000 & 0.000 & .500 & .500 \\ \text { DRVE } & 0.000 & 18.000 & 0.000 & 0.000 & 4.500 & 4.500 \\ \text { FEOC } & 0.000 & 4.000 & 0.000 & 12.000 & 4.000 & 2.828 \\ \text { TRDU } & 0.000 & 2.000 & 0.000 & 0.000 & .500 & .500 \\ \text { BBR } & 0.000 & 10.000 & 0.000 & -0.000 & 2.500 & 2.500 \\ \text { SALX } & 0.000 & 0.000 & 0.000 & 1.500 & 1.500 \\ \text { LBR } & 0.000 & 6.000 & 0.000 & 0.000 & 1.500 & 1.500 \\ \text { ASTR } & 0.000 & 0.000 & 0.000 & 10.000 & 4.000 & 2.449 \\ \text { ACMI } & 0.000 & 0.000 & 0.000 & .500 & .500 \\ \text { TOTLD } & 0.000 & 2.000 & 1.000 \\ \text { POSE } & 0.000 & 0.000 & 100.000 & 96.000 & 49.000 & 28.302 \\ \text { BRTED } & 0.000 & 0.000 & 72.000 & 2.000 & 18.500 & 17.840 \\ \text { ATSP } & 0.000 & 0.000 & 98.000 & 0.000 & 24.500 & 24.500 \\ \text { DEPI } & 0.000 & 0.000 & 18.000 & 0.000 & 4.500 & 4.500 \\ \text { AMAC } & 0.000 & 0.000 & 0.000 & 30.000 & 7.500 & 7.500 \\ \text { AMLY } & 0.000 & 0.000 & 0.000 & 22.000 & 5.500 & 5.500 \\ \text { ERAS } & 0.000 & 0.000 & 0.000 & 2.000 & .500 & .500 \\ \text { ARTR } & 0.000 & 0.000 & 0.000 & 6.000 & 1.500 & 1.500 \\ & 0.000 & 0.000 & 0.000 & 14.000 & 3.500 & 3.500\end{array}$




\section{Computer Program for Summary of Vegetation Data}

PROGRAM COVER (INPUT, OUTPUT, CARDS, TAPES5=1NPUT, TAPE6=OUTPUT, + TAPE8=CARDS) DIMENSION TITLE (12)

DIMENSION COV $(50,12)$, FREQ $(50,12)$, CODE (7) NAME (50,) KARD (50)

DIMENSION SSX $(50,12)$, SSXX $(50,12)$ SD $(50,12)$, SE $(50,12), C V(50,12)$

DIMENSION CI95U (12), CI95L (12)

DIMENSION ISTNM (7)

COMMON /A/ LINES

DIMENSION TOLIN $(50,12)$

COVER CALCULATES SEVERAL STATISTICS ON EACH SPECIES AND LINE, AND DIVERSITY INDICES OF HILL ECOL. 1973. THE

DATA ARE IN GROUPS OF LINES. THE NUMBER OF PLOTS PER LINE AND TITLES FOR EACH LINE PRECEED EACH GROUP. DATA FOR A LINE IS FOLLOWED BY A 9999 CARD, EXCEPT THE LAST. GROUPS ARE FOLLOWED BY A \$\$\$ CARD, EXCEPT THE LAST.

IN THE ARRAYS, 50 REFS THE SPECIES EXCEPT IN TOLIN WHERE 50 REFS TO THE NUMBER OF PLOTS PER LINE. 12 REFS TO THE MAX NUMBER OF LINES PER GROUP (10) PLUS ONE FOR MEAN AND ONE FOR SE.

BY RONALD H. SAUER DEC 1976

DATA CODE/0., 2.5., 15., 37.5, 62.5, 85., 97.5/

DATA ISTNM/5HSUM X,5HS $X * X, 5 H S D \quad, 5 H C V \quad, 5 H S E \quad, 5 H 95 \% U, 5 H 95 \% \mathrm{~L}$ $+1$

20 CONTINUE

DO $30 \mathrm{I}=1,50$

DO $30 \mathrm{~J}=1,12$

$\operatorname{COV}(I, J)=0$.

$\operatorname{FREQ}(1, J)=0$.

$\operatorname{SSX}(1, J)=0$.

SSXX $(1, J)=0$.

$\mathrm{SD}(1, J)=0$.

$\mathrm{CV}(1, \mathrm{~J})=0$.

SE $(I, J)=0$.

TOLIN $(1, J)=0$.

CONTINUE

40

DO $40 \mathrm{~J}=1,50$

$c$ NAME $(J)=4 \mathrm{H}$ NSPS $=0$

READ THE NUMBER OF PLOTS IN EACH LINE IN CLRRENT GROUP READ $(5,50)$ NPLTS

FORMAT (13)

60 FORMAT $(1 \mathrm{H} 1$, * THE NUMBER OF PLOTS IN THE CURRENT GROUP $=$ *, 1 13)

READ THE TITLES FOR EACH GROUP ( 8 CHARACTERS LONG)

$80 \operatorname{READ}(5,90)$ KSP.M.MM,MMM,KARD

FORMAT (A5.A9,.A10.46.5011)

IF (EOF (5). NE. 0) GO TO 150 


\section{Computer Program for Summary of Vegetation Data}

60

C

65

70

75

80

85

90

95

100

105

C

IF (KSP.EQ.5H9999 ) $L=L+1$

IF (L.GT.10) GO TO 150

IF (KSP.EQ.5H9999, GO TO 80

CATALOG SPECIES NUMBER ID OR DETERMINE ID NUMBER IF SPECIES PREVIOUSLY CATALOGED

IF (NSPS.EQ.0) GO TO 120

DO $110 \mathrm{~K}=$, NSPS

$K P=K$

If (KSP.EQ.NAME (K)) GO TO 130

110 CONTINUE

120 CONTINUE

$N S P S=N S P S+1$

NAME (NSPS) $=K S P$

$K P=N S P S$

130 CONTINUE

$S X=S X X=F=0$.

DO $140 K=1$, NPLTS

If $(K A R D(K) \cdot E Q .0) \quad K A R D(K)=0$

I=KARD $(K)+1$

IF (I.GT.1) $\quad F=F+1$

TEMP $=$ CODE $(I)$

C SUM ALL SPECIES IN PLOT K, LINE L INTO TOLIN(K,L)

TOLIN $(K, L)=$ TOLIN $(K, L)+$ TEMP

$S X=S X+T E M P$

$S X X=S X X+T E M P * T E M P$

140 CONTINUE

FREQ $(K P, L)=F * 100 . / N P L T S$

$\operatorname{COV}(K P, L)=S X / N P L T S$

$S S X(K P, L)=S X$

$\operatorname{SSXX}(K P, L)=S X X$

$H=N P L T S$

TEMP $=S X X-S X * S X / H$

$S D(K P, L)=S Q R T(T E M P /(H-1))$

$C V(K P, L)=S D(K P, L) / C O V(K P, L)$

$S E(K P, L)=S D(K P, L) / S Q R T(H)$

GO TO 80

150 CONTINLE

LINES $=\mathrm{L}$

NAME (NSPS 1$)=5$ HTOTAL

NAME (NSPS+2) $=5 H N(0)$

NAME (NSPS+3) $=5 \mathrm{HN}(1)$

NAME (NSPS+4)=5HN (2)

TITLE (LINES+1) $=8 \mathrm{H}$ MEAN

TITLE (LINES +2 ; $=8$ HSTAN ERR

$J=L I N E S+2$

$11=N S P S+1$

$12=N S P S+2$

$13=N S P S+3$

$14=N S P S+4$

TOTAL COVER FOR EACH PLOT

DO 170 L $=1$, LINES

$5 X=0$.

$110 \quad 5 X X=0$

DO 160 JKS $=1$, NPLTS

TEMP $=$ TOLIN(JKS,L) 


\section{Computer Program for Summary of Vegetation Data}

250
$S X=S X+T E M P$

$S X X=S X X+T E M P *$ TEMP

CONTINUE

$\operatorname{SSX}(17, L)=S X$

$\operatorname{SSXX}(11, L)=S X X$

$H=N P L T S$

$T E M P=S X X-S X * S X / H$

SD $(I 1, \mathrm{~L})=S Q R T(T E M P /(H-1)$.

CV $(11, \mathrm{~L})=S D(11, L) /(S X / H)$

SE $(11, L)=S D(11, L) / S Q R T(H)$

TOTAL ALL SPECIES IN A GROUP. DO $180 \mathrm{~L}=1$, LINES

DO $180 \mathrm{~K}=1$, NSPS

$\operatorname{COV}(11, L)=\operatorname{COV}(11, L)+\operatorname{COV}(K, L)$

CALCLLATE MEAN AND STANDARD ERROR FOR PERCENT COVER FOR EACH SPECIES CALL STER (COV,1,I1)

CALL STER $($ SSX,1,I1)

CALL STER (SSXX,1,11)

CALL STER (SD,1,11)

CALL STER (CV,1,11)

CALL STER (SE,1,11)

PRINT THE COVER PERCENTS

WRITE $(6,190)$ (TITLE (I), $\{=1, J)$

190 FORMAT $(1 \mathrm{H} 1, \mathrm{~T} 50 . *$ PERCENT COVER BY SPECIES $*, / 1,10 \mathrm{X}, 12 \mathrm{~A} 10)$ DO $220 \mathrm{~K}=\mathrm{a}, 11$

WRITE $(6,240)$ NAME (K), (COV $(K, L), L=1, J)$

WRITE $(6,200)$ ISTNM (1), (SSX(K,L),L=1,J))

WRITE $(6,200)$ ISTNM (2), (S5XX(K,L),L=1,J))

FORMAT $(2 \mathrm{H}, A 6,12 \mathrm{~F} 10.1)$

WRITE $(6,230)$ ISTNM (3), (SD(K,L),L=1,J)

WRITE $(6,230)$ ISTNM (4), (CViK,L),L=J))

WRITE $(6,230)$ ISTNM (5), (SE(K,L),L=1,J)

DO 210$\rfloor K=1, J]$

CI95L $(J K)=\operatorname{COV}(K, J K)+S E(K, J K) * 1.910$

CI95L $($ IK $)=\operatorname{COV}^{\prime}(K, J K)-S E(K, J K) * 1.910$

CONTINUE

WRITE (6.230) ISTNM (6), (CI95L(LL),LL=1,J)

WRITE $(6,230)$ ISTNM (7), (CI95L (LL),LL=1,J)

CONTINUE

FORMAT $(2 \mathrm{H}, A 6.12 \mathrm{~F} 10.3)$

FORMAT (1H ,A6,12F10.3)

CHANGE \% COVER TO RELATIVE COVER.

DO $250 \mathrm{~L}=1$. LINES

DO $250 \mathrm{~K}=1$, NSPS

$\operatorname{COV}(K, L)=\operatorname{COV}(K, L) / \operatorname{COV}(11, L)$

CALCLLATE DIVERSITY INDICES ACCORDING TO HILL IN 1973 ECOLOGY

$N(A)=(S U M$ OF $P * * A) * *(1 .,(1 .-A))$

$A=0$ IS NUMABER OF SPECIES.

$A=1$ IS EXP (SHANNONS ENTROPY)

$A=2$ IS $1 /$ SIMPSONS MODIFIED INDEX.

$A=0$ OR $N(O)$

$A=0$

DO $270 L=1$, LINES

SUN $1=0$

DO $260 k=1$. $\checkmark S P S$

CONTINLE 


\section{Computer Program for Summary of Vegetation Data}

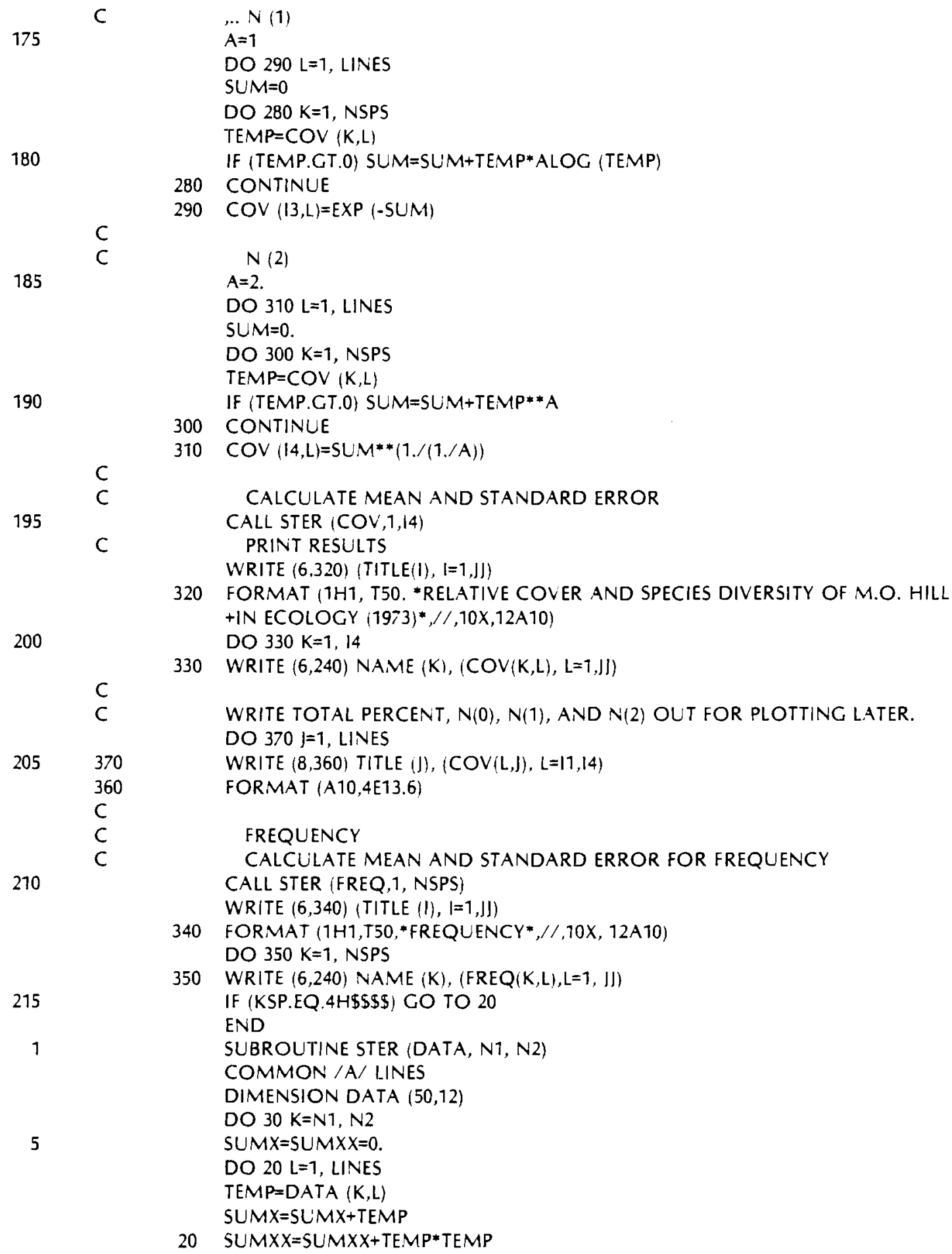

WRITE TOTAL PERCENT, N(0), N(1), AND N(2) OUT FOR PLOTTING LATER. DO $370 \mathrm{j}=1$, LINES 


\section{Computer Program for Summary of Vegetation Data}

10

15

SE $2=($ SUMXX- $($ SUMX*SUMX) $/$ LINES $) /($ LINES* $($ LINES- 1$)$

$S E=S Q R T$ (SE2)

$A V E=S U M X / L I N E S$

DATA $(K$, LINES +1$)=$ AVE

DATA $(K, L I N E S+2)=S E$

30 CONTINUE

RETURN

END

$A-18$ 
APPENDIX B

DEER DIETS BY PELLET GROUP COLLECTED

AT B-C CRIBS, B POND AND GABLE MOUNTAIN POND HABITATS 
Plant Codes Used for 1976 Deer Samples for Diet Analyses:

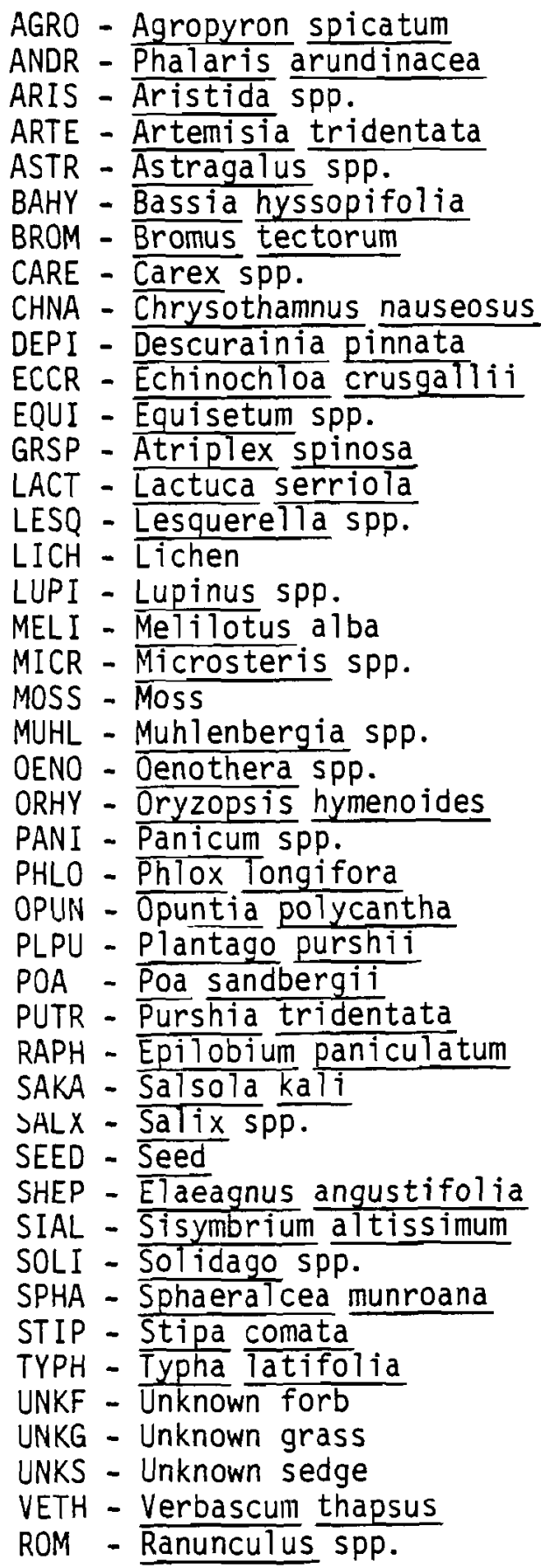


Codes Used for Site (Transect) with Corresponding Diet and Radiation Sample Numbers and Age of Deer Pellet Groups Collected on the Hanford Reservation During 1976 (contd)

B POND

\begin{tabular}{|c|c|c|c|c|}
\hline Site & $\begin{array}{l}\text { Diet } \\
\text { No. } \\
\end{array}$ & $\begin{array}{l}\text { Radiation } \\
\text { No. } \\
\end{array}$ & Age & Date Collected \\
\hline $\begin{array}{l}B P 1 \\
\text { BP1 } \\
\text { BP1 } \\
\text { BP1 } \\
\text { BP1 } \\
\text { BP1 } \\
\text { BP1 } \\
\text { BP 1 } \\
\text { BP1 } \\
\text { BP1 }\end{array}$ & $\begin{array}{l}001 \\
002 \\
003 \\
004 \\
005 \\
006 \\
007 \\
008 \\
009 \\
010\end{array}$ & $\begin{array}{l}467 \\
470 \\
464 \\
463 \\
468 \\
461 \\
462 \\
466 \\
469 \\
465\end{array}$ & $\begin{array}{l}\text { Recent } \\
\text { 0ld } \\
\text { Fresh } \\
\text { 01d } \\
\text { 01d } \\
\text { Recent } \\
\text { Fresh } \\
\text { Recent } \\
\text { Fresh } \\
\text { Recent }\end{array}$ & $\begin{array}{l}7-30-76 \\
7-30-76 \\
7-30-76 \\
7-30-76 \\
7-30-76 \\
7-30-76 \\
7-30-76 \\
7-30-76 \\
7-30-76 \\
7-30-76\end{array}$ \\
\hline $\begin{array}{l}\text { BP2 } \\
\text { BP2 } \\
\text { BP2 } \\
\text { BP2 } \\
\text { BP2 } \\
\text { BP2 } \\
\text { BP2 } \\
\text { BP2 } \\
\text { BP2 } \\
\text { BP2 }\end{array}$ & $\begin{array}{l}011 \\
012 \\
013 \\
014 \\
015 \\
016 \\
017 \\
018 \\
019 \\
020\end{array}$ & $\begin{array}{l}478 \\
474 \\
479 \\
471 \\
476 \\
477 \\
473 \\
480 \\
475 \\
472\end{array}$ & $\begin{array}{l}\text { 01d } \\
\text { 01d } \\
\text { Fresh } \\
\text { 01d } \\
\text { Fresh } \\
\text { Fresh } \\
\text { Recent } \\
\text { Recent } \\
\text { Recent } \\
\text { 01d }\end{array}$ & $\begin{array}{l}7-30-76 \\
7-30-76 \\
7-30-76 \\
7-30-76 \\
7-30-76 \\
7-30-76 \\
7-30-76 \\
7-30-76 \\
7-30-76 \\
7-30-76\end{array}$ \\
\hline $\begin{array}{l}\text { BP3 } \\
\text { BP3 } \\
\text { BP3 } \\
\text { BP3 } \\
\text { BP3 } \\
\text { BP3 } \\
\text { BP3 } \\
\text { BP3 } \\
\text { BP3 } \\
\text { BP3 }\end{array}$ & $\begin{array}{l}021 \\
022 \\
023 \\
024 \\
025 \\
026 \\
027 \\
028 \\
029 \\
030\end{array}$ & $\begin{array}{l}486 \\
484 \\
482 \\
489 \\
488 \\
487 \\
481 \\
490 \\
483 \\
485\end{array}$ & $\begin{array}{l}\text { Recent } \\
\text { Recent } \\
\text { Recent } \\
\text { Recent } \\
\text { Recent } \\
\text { Recent } \\
\text { Recent } \\
\text { Recent } \\
\text { Recent } \\
\text { 0ld }\end{array}$ & $\begin{array}{l}7-30-76 \\
7-30-76 \\
7-30-76 \\
7-30-76 \\
7-30-76 \\
7-30-76 \\
7-30-76 \\
7-30-76 \\
7-30-76 \\
7-30-76\end{array}$ \\
\hline $\begin{array}{l}\text { BP4 } \\
\text { BP4 } \\
\text { BP4 } \\
\text { BP4 } \\
\text { BP4 } \\
\text { BP4 } \\
\text { BP4 } \\
\text { BP4 } \\
\text { BP4 } \\
\text { BP4 }\end{array}$ & $\begin{array}{l}031 \\
032 \\
033 \\
034 \\
035 \\
036 \\
037 \\
038 \\
039 \\
040\end{array}$ & $\begin{array}{l}493 \\
498 \\
497 \\
499 \\
496 \\
492 \\
495 \\
500 \\
494 \\
491\end{array}$ & $\begin{array}{l}\text { 01d } \\
\text { Recent } \\
\text { Recent } \\
\text { 01d } \\
\text { 01d } \\
\text { Recent } \\
\text { Recent } \\
\text { Recent } \\
\text { 01d } \\
\text { Recent }\end{array}$ & $\begin{array}{l}7-30-76 \\
7-30-76 \\
7-30-76 \\
7-30-76 \\
7-30-76 \\
7-30-76 \\
7-30-76 \\
7-30-76 \\
7-30-76 \\
7-30-76\end{array}$ \\
\hline
\end{tabular}


Codes Used for Site (Transect) with Corresponding Diet and Radiation Sample Numbers and Age of Deer Pellet Groups Collected on the Hanford Reservation During 1976 (contd)

GABLE MOUNTAIN POND

\begin{tabular}{|c|c|c|c|c|}
\hline Site & $\begin{array}{l}\text { Diet } \\
\text { No. } \\
\end{array}$ & $\begin{array}{c}\text { Radiation } \\
\text { No. } \\
\end{array}$ & Age & Date Collected \\
\hline $\begin{array}{l}\text { GM1 } \\
\text { GM1 } \\
\text { GM1 } \\
\text { GM1 } \\
\text { GM1 } \\
\text { GM1 } \\
\text { GM1 } \\
\text { GM1 } \\
\text { GM1 } \\
\text { GM1 }\end{array}$ & $\begin{array}{l}041 \\
042 \\
043 \\
044 \\
045 \\
046 \\
047 \\
048 \\
049 \\
050\end{array}$ & $\begin{array}{l}402 \\
410 \\
404 \\
403 \\
405 \\
407 \\
401 \\
409 \\
406 \\
408\end{array}$ & $\begin{array}{l}\text { Recent } \\
\text { old } \\
\text { old } \\
\text { old } \\
\text { old } \\
\text { old } \\
\text { old } \\
\text { old } \\
\text { Recent } \\
\text { old }\end{array}$ & $\begin{array}{l}7-29-76 \\
7-29-76 \\
7-29-76 \\
7-29-76 \\
7-29-76 \\
7-29-76 \\
7-29-76 \\
7-29-76 \\
7-29-76 \\
7-29-76\end{array}$ \\
\hline $\begin{array}{l}\text { GM2 } \\
\text { GM2 } \\
\text { GM2 } \\
\text { GM2 } \\
\text { GM2 } \\
\text { GM2 } \\
\text { GM2 } \\
\text { GM2 } \\
\text { GM2 } \\
\text { GM2 }\end{array}$ & $\begin{array}{l}051 \\
052 \\
053 \\
054 \\
055 \\
056 \\
057 \\
058 \\
059 \\
060\end{array}$ & $\begin{array}{l}412 \\
415 \\
420 \\
418 \\
413 \\
411 \\
417 \\
419 \\
416 \\
414\end{array}$ & $\begin{array}{l}\text { 01d } \\
\text { 01d } \\
\text { Recent } \\
\text { 01d } \\
\text { Recent } \\
\text { Recent } \\
\text { 01d } \\
\text { 01d } \\
\text { old } \\
\text { 01d }\end{array}$ & $\begin{array}{l}7-29-76 \\
7-29-76 \\
7-29-76 \\
7-29-76 \\
7-29-76 \\
7-29-76 \\
7-29-76 \\
7-29-76 \\
7-29-76 \\
7-29-76\end{array}$ \\
\hline $\begin{array}{l}\text { GM3 } \\
\text { GM3 } \\
\text { GM3 } \\
\text { GM3 } \\
\text { GM3 } \\
\text { GM3 } \\
\text { GM3 } \\
\text { GM3 } \\
\text { GM3 } \\
\text { GM3 }\end{array}$ & $\begin{array}{l}061 \\
062 \\
063 \\
064 \\
065 \\
066 \\
067 \\
068 \\
069 \\
070\end{array}$ & $\begin{array}{l}430 \\
424 \\
428 \\
429 \\
425 \\
423 \\
426 \\
421 \\
427 \\
422\end{array}$ & $\begin{array}{l}\text { 0ld } \\
\text { Fresh } \\
\text { old } \\
\text { old } \\
\text { Recent } \\
\text { Recent } \\
\text { Recent } \\
\text { Recent } \\
\text { Old } \\
\text { Recent }\end{array}$ & $\begin{array}{l}7-29-76 \\
7-29-76 \\
7-29-76 \\
7-29-76 \\
7-29-76 \\
7-29-76 \\
7-29-76 \\
7-29-76 \\
7-29-76 \\
7-29-76\end{array}$ \\
\hline $\begin{array}{l}\text { GM4 } \\
\text { GM4 } \\
\text { GM4 } \\
\text { GM4 } \\
\text { GM4 } \\
\text { GM4 } \\
\text { GM4 } \\
\text { GM4 } \\
\text { GM4 } \\
\text { GM4 }\end{array}$ & $\begin{array}{l}071 \\
072 \\
073 \\
074 \\
075 \\
076 \\
077 \\
078 \\
079 \\
080\end{array}$ & $\begin{array}{l}433 \\
438 \\
436 \\
435 \\
431 \\
437 \\
439 \\
440 \\
434 \\
432\end{array}$ & $\begin{array}{l}\text { 01d } \\
\text { Fresh } \\
\text { Recent } \\
\text { Recent } \\
\text { Recent } \\
\text { Recent } \\
\text { old } \\
\text { Old } \\
\text { Recent } \\
\text { 0ld }\end{array}$ & $\begin{array}{l}7-30-76 \\
7-30-76 \\
7-30-76 \\
7-30-76 \\
7-30-76 \\
7-30-76 \\
7-30-76 \\
7-30-76 \\
7-30-76 \\
7-30-76\end{array}$ \\
\hline
\end{tabular}


Codes Used for Site (Transect) with Corresponding Diet and Radiation Sample Numbers and Age of Deer Pellet Groups

Collected on the Hanford Reservation During 1976

\section{$\underline{B-C}$ CRIBS}

\begin{tabular}{|c|c|c|c|c|}
\hline Site & $\begin{array}{l}\text { Diet } \\
\text { No. } \\
\end{array}$ & $\begin{array}{c}\text { Radiation } \\
\text { No. }\end{array}$ & Age & Date Collected \\
\hline $\begin{array}{l}B C 1 \\
B C 1 \\
B C 1 \\
B C 1 \\
B C 1 \\
B C 1 \\
B C 1 \\
B C 1 \\
B C 1 \\
B C 1\end{array}$ & $\begin{array}{l}081 \\
082 \\
083 \\
084 \\
085 \\
086 \\
087 \\
088 \\
089 \\
090\end{array}$ & $\begin{array}{l}448 \\
444 \\
445 \\
443 \\
450 \\
446 \\
441 \\
447 \\
449 \\
442\end{array}$ & $\begin{array}{l}\text { 01d } \\
\text { 01d } \\
\text { Recent } \\
\text { 01d } \\
\text { Recent } \\
\text { Recent } \\
\text { Recent } \\
\text { 01d } \\
\text { 01d } \\
\text { Recent }\end{array}$ & $\begin{array}{l}8-4-76 \\
8-4-76 \\
8-4-76 \\
8-4-76 \\
8-4-76 \\
8-4-76 \\
8-4-76 \\
8-4-76 \\
8-4-76 \\
8-4-76\end{array}$ \\
\hline $\begin{array}{l}\mathrm{BC} 2 \\
\mathrm{BC2} \\
\mathrm{BC} 2 \\
\mathrm{BC} 2 \\
\mathrm{BC} 2 \\
\mathrm{BC} 2 \\
\mathrm{BC} 2 \\
\mathrm{BC} 2 \\
\mathrm{BC} 2 \\
\mathrm{BC2}\end{array}$ & $\begin{array}{l}091 \\
092 \\
093 \\
094 \\
095 \\
096 \\
097 \\
098 \\
099 \\
100\end{array}$ & $\begin{array}{l}453 \\
452 \\
451 \\
456 \\
460 \\
454 \\
459 \\
457 \\
458\end{array}$ & $\begin{array}{l}\text { 01d } \\
\text { 01d } \\
\text { Recent } \\
\text { 01d } \\
\text { 01d } \\
\text { 01d } \\
\text { Recent } \\
\text { 01d } \\
\text { 01d } \\
\text { 01d }\end{array}$ & $\begin{array}{l}8-4-76 \\
8-4-76 \\
8-4-76 \\
8-4-76 \\
8-4-76 \\
8-4-76 \\
8-4-76 \\
8-4-76 \\
8-4-76 \\
8-4-76\end{array}$ \\
\hline
\end{tabular}


DIET ANALYSIS FOR CONSUMER ODHE ON DATE 073076 WITH 100. OBSERVED FIELDS, I SLIDES AND 4 FOOD SPECIES

$\begin{array}{ccc}\text { BP-1 } & & \text { RADIATION } \\ \text { DIET SAMPLE } & & \begin{array}{c}\text { SAMPLE NO. } \\ 467\end{array} \\ 1 & & \text { DENSITY } \\ \text { FOOD SPECIES } & \text { FREQUENCY } & .1393 \\ \text { SALX } & 13.000 & 1.3093 \\ \text { SAKA } & 73.0000 & .0726 \\ \text { ECCR } & 7.0000 & .0305 \\ \text { CHNA } & 3.0000 & \\ & & \\ \text { FOOD SPECIES } & \text { REL FREQ } & \text { REL DENS } \\ \text { SALX } & 13.5417 & 8.9752 \\ \text { SAKA } & 76.0417 & 84.3846 \\ \text { ECCR } & 7.2917 & 4.5771 \\ \text { CHNA } & 3.1250 & 1.9631\end{array}$

DIET ANALYSIS FOR CONSLMER ODHE ON DATE 073076 WITH 100. OBSERVED FIELDS, 5 SLIDES AND 5 FOOD SPECIES

$\begin{array}{lcc}\begin{array}{c}\text { BP-1 } \\ \text { DIET SAMPLE }\end{array} & & \text { RADIATION } \\ 3 & & \text { SAMPLE NO. } \\ 4 & & 464 \\ \text { FOOD SPECIES } & \text { FREQUENCY } & \text { DENSITY } \\ \text { SAKA } & 97.0000 & 3.5066 \\ \text { ECCR } & 3.0000 & .0305 \\ \text { BROM } & 1.0000 & .0101 \\ \text { SEED } & 4.0000 & .0408 \\ \text { POA } & 1.0000 & .0101 \\ & & \\ \text { FOOD SPECIES } & \text { REL FREQ } & \text { REL DENS } \\ \text { SAKA } & 91.5094 & 97.4602 \\ \text { ECCR } & 2.8302 & .8466 \\ \text { BROM } & .9434 & .2793 \\ \text { SEED } & 3.7736 & 1.1346 \\ \text { POA } & .9434 & .2793\end{array}$

DIET ANALYSIS FOR CONSUMER ODHE ON DATE 073076 WITH 100. OBSERVED FIELDS, 5 SLIDES AND 8 FOOD SPECIES

\begin{tabular}{llc}
$\begin{array}{c}\text { BP-1 } \\
\text { DIET SAMPLE }\end{array}$ & & RADIATION \\
\multicolumn{2}{c}{ SAMPLE NO. } \\
FOOD SPECIES & FREQUENCY & 470 \\
SAKA & 80.0000 & 1.6094 \\
SOUI & 35.0000 & .4308 \\
SEED & 5.0000 & .0513 \\
OENO & 1.0000 & .0101 \\
SHEP & 1.0000 & .0101 \\
BROM & 1.0000 & .0101 \\
SPHA & 1.0000 & .0101 \\
ECCR & 2.0000 & .0202 \\
& & \\
FOOD SPECIES & REL FREQ & REL DENS \\
SAKA & 63.4921 & 74.7909 \\
SOLI & 27.7778 & 20.0186 \\
SEED & 3.9683 & 2.3836 \\
OENO & .7937 & .4670 \\
SHEP & .7937 & .4670 \\
BROM & .7937 & .4670 \\
SPHA & .7937 & .4670 \\
ECCR & 1.5873 & .9388
\end{tabular}

DIET ANALYSIS FOR CONSUMER ODHE ON DATE 073076 WITH 100. OBSERVED FIELDS, 5 SLIDES AND 6 FOOD SPECIES

$\begin{array}{lcc}\begin{array}{c}\text { BP-1 } \\ \text { DIET SAMPLE }\end{array} & & \begin{array}{c}\text { RADIATION } \\ \text { SAMPLE NO. } \\ 463\end{array} \\ \text { FOOD SPECIES } & \text { FREQUENCY } & \text { DENSITY } \\ \text { SAKA } & 88.0000 & 2.1203 \\ \text { SOLI } & 30.0000 & .3567 \\ \text { ECCR } & 5.0000 & .0513 \\ \text { BROM } & 3.0000 & .0305 \\ \text { SALX } & 4.0000 & .0408 \\ \text { SEED } & 1.0000 & .0101 \\ & & \\ \text { FOOD SPECIES } & \text { REL FREQ } & \text { REL DENS } \\ \text { SAKA } & 67.1756 & 31.2497 \\ \text { SOLI } & 22.9608 & 13.6620 \\ \text { ECCR } & 3.8168 & 1.9656 \\ \text { BROM } & 2.2901 & 1.1672 \\ \text { SALX } & 3.0534 & 1.5643 \\ \text { SEED } & .7634 & .3851\end{array}$


DIET ANALYSIS FOR CONSUMER ODHE ON DATE 073070́ WITH 100. OBSERVED FIELDS, 5 SLIDES AND 4 FOOD SPECIES

$\begin{array}{ccc}\text { BP-1 } & & \text { RADIATION } \\ \text { DIET SAMPLE } & & \text { SAMPLE NO. } \\ 5 & & 468 \\ \text { FOOD SPECIES } & \text { FREQUENCY } & \text { DENSITY } \\ \text { SAKA } & 96.000 & 3.2189 \\ \text { ECCR } & 3.0000 & .0305 \\ \text { SPHA } & 8.0000 & .0834 \\ \text { SOLI } & 2.0000 & .0202 \\ & & \\ \text { FOOD SPECIES } & \text { REL FREQ } & \text { REL DENS } \\ \text { SAKA } & 88.0734 & 96.0022 \\ \text { ECCR } & 2.7523 & .9084 \\ \text { SPHA } & 7.3394 & 2.4868 \\ \text { SOLI } & -7.8349 & .6025\end{array}$

DIET ANALYSIS FOR CONSUMER ODHE ON DATE 073076 WITH 100. OBSERVED FIELDS, 5 SLIDES AND 5 FOOD SPECIES

$\begin{array}{lcc}\text { BP-1 } & & \text { RADIATION } \\ \text { DIET SAMPLE } & & \text { SAMPLE NO. } \\ 7 & & 462 \\ \text { FOOD SPECIES } & \text { FREQUENCY } & \text { DENSITY } \\ \text { VETH } & 75.0000 & 1.3863 \\ \text { SAKA } & 75.0000 & 1.3863 \\ \text { SOLI } & 50.0000 & .6931 \\ \text { ECCR } & 9.0000 & .0943 \\ \text { SEED } & 2.0000 & .0202 \\ & & \\ \text { FOOD SPECIES } & \text { REL FREQ } & \text { REL DENS } \\ \text { VETH } & 35.5450 & 38.7206 \\ \text { SAKA } & 35.5450 & 38.7206 \\ \text { SOLI } & 23.6967 & 19.3603 \\ \text { ECCR } & 4.2654 & 2.6342 \\ \text { SEED } & .9479 & .5643\end{array}$

DIET ANALYSIS FOR CONSUMER ODHE ON DATE 073076 WITH 100. OBSERVED FIELDS, 5 SLIDES AND 6 FOOD SPECIES

$\begin{array}{lcc}\text { BP-1 } & & \text { RADIATION } \\ \text { DIET SAMPLE } & & \begin{array}{c}\text { SAMPLE NO. } \\ 6\end{array} \\ \text { FOOD SPECIES } & \text { FREQUENCY } & \text { DENSITY } \\ \text { SOLI } & 38.0000 & .4780 \\ \text { SAKA } & 68.0000 & 1.1394 \\ \text { SALX } & 10.0000 & .1054 \\ \text { ECCR } & 15.0000 & .1625 \\ \text { BROM } & 4.0000 & .0408 \\ \text { SEED } & 2.0000 & .0202 \\ & & \\ \text { FOOD SPECIES } & \text { REL FREQ } & \text { REL DENS } \\ \text { SOLI } & 27.7372 & 24.5603 \\ \text { SAKA } & 49.6350 & 58.5414 \\ \text { SALX } & 7.2993 & 5.4132 \\ \text { ECCR } & 10.9489 & 8.3498 \\ \text { BROM } & 2.9197 & 2.0973 \\ \text { SEED } & 1.4599 & 1.0380\end{array}$

DIET ANALYSIS FOR CONSUMER ODHE ON DATE 073076 WITH 100. OBSERVED FIELDS, 5 SLIDES AND 6 FOOD SPECIES

\begin{tabular}{lcc} 
BP-1 & & RADIATION \\
DIET SAMPLE & & SAMPLE NO. \\
\multicolumn{1}{c}{8} & & 466 \\
FOOD SPECIES & FREQUENCY & DENSITY \\
SAKA & 75.0000 & 1.3863 \\
SEED & 8.0000 & .0834 \\
BROM & 19.0000 & .2107 \\
ECCR & 25.0000 & .2877 \\
SOLI & 9.0000 & .0943 \\
SALX & 3.0000 & .0305 \\
& & \\
FOOD SPECIES & REL FREQ & REL DENS \\
SAKA & 53.9568 & 66.2396 \\
SEED & 5.7554 & 3.9841 \\
BROM & 13.6691 & 10.0686 \\
ECCR & 17.9856 & 13.7460 \\
SOLI & 6.4748 & 4.5063 \\
SALX & 2.1583 & 1.4554
\end{tabular}


DIET ANALYSIS FOR CONSUMER ODHE ON DATE 073076 WITH 100. OBSERVED FIELDS, 5 SLIDES AND 3 FOOD SPECIES

$B P-1$

DIET SAMPLE

9

FOOD SPECIES

SAKA

ECCR

SOLI

FOOD SPECIES

SAKA

ECCR

SOLI

RADIATION
SAMPLE NO.
469
DENSITY
3.5066
.0408
.0101
REL DENS
98.5700
1.1475
.2825

DIET ANALYSIS FOR CONSUMER ODHE ON DATE 073076 WITH 100. OBSERVED FIELDS, 5 SLIDES AND 6 FOOD SPECIES

$\begin{array}{lcc}\text { BP-1 } & & \text { RADIATION } \\ \text { DIET SAMPLE } & & \begin{array}{c}\text { SAMPLENO } \\ 10\end{array} \\ \text { FOOD SPECIES } & \text { FREQUENCY } & \text { DENSITY } \\ \text { SALX } & 42.0000 & .5447 \\ \text { ECCR } & 30.0000 & .3567 \\ \text { SAKA } & 45.0000 & .5978 \\ \text { SOLI } & 9.0000 & .0943 \\ \text { VETH } & 1.0000 & .0101 \\ \text { BROM } & 1.0000 & .0101 \\ & & \\ \text { FOOD SPECIES } & \text { REL FREQ } & \text { REL DENS } \\ \text { SALY } & 32.8125 & 33.7574 \\ \text { ECCR } & 23.4375 & 22.1036 \\ \text { SAKA } & 35.1563 & 37.0487 \\ \text { SOLI } & 7.0313 & 5.8446 \\ \text { VETH } & .7813 & .6228 \\ \text { BROM } & .7813 & .6228\end{array}$

DIET ANALYSIS FOR CONSUMER ODHE ON DATE 073076 WITH 100. OBSERVED FIELDS, 5 SLIDES AND 6 FOOD SPECIES

$\begin{array}{llc}\text { BP-2 } & & \text { RADIATION } \\ \text { DIET SAMPLE } & & \text { SAMPLE NO. } \\ 11 & & 478 \\ \text { FOOD SPECIES } & \text { FREQUENCY } & \text { DENSITY } \\ \text { SPHA } & 95.0000 & 2.9957 \\ \text { SOLI } & 14.0000 & .1508 \\ \text { SEED } & 6.0000 & .0619 \\ \text { SAKA } & 9.0000 & .0943 \\ \text { ECCR } & 3.0000 & .0305 \\ \text { POA } & 1.0000 & .0101 \\ & & \\ \text { FOOD SPECIES } & R E L F R E Q & \text { REL DEVS } \\ \text { SPHA } & 74.2138 & 89.5054 \\ \text { SOLI } & 10.9375 & 4.5113 \\ \text { SEED } & 4.6875 & 1.8508 \\ \text { S.AK } & 7.0313 & 2.5209 \\ \text { ECCR } & 2.3438 & .9111 \\ \text { POA } & .7813 & .3006\end{array}$

DIET ANALYSIS FOR CONSUMER ODHE ON DATE 073076 WITH 100. OBSERVED FIELDS, 5 SLIDES AND 7 FOOD SPECIES

$\begin{array}{ccc}\text { BP-2 } & & \text { RADIATION } \\ \text { DiET SAMPLE } & & \text { SAMPLE NO. } \\ 12 & & 474 \\ \text { FOOD SPECIES } & \text { FREQUENCY } & \text { DENSITY } \\ \text { SOLI } & 15.0000 & .1625 \\ \text { SALX } & 9.0000 & .0943 \\ \text { SAKA } & 75.0000 & 1.3863 \\ \text { SEED } & 14.0000 & .1508 \\ \text { SPHA } & 5.0000 & .0513 \\ \text { BROM } & 10.0000 & .1054 \\ \text { OENO } & 2.0000 & .0202 \\ & & \\ \text { FOOD SPECIES } & R E L ~ F R E Q & \text { REL DENS } \\ \text { SOLI } & 11.5385 & 8.2463 \\ \text { SALX } & 6.9231 & 4.7854 \\ \text { SAKA } & 57.6923 & 70.3416 \\ \text { SEED } & 10.7692 & 7.6529 \\ \text { SPHA } & 3.8462 & 2.6027 \\ \text { BROM } & 7.6923 & 5.3461 \\ \text { OENO } & 1.5385 & 1.0251\end{array}$


DIET ANALYSIS FOR CONSUMER ODHE ON DATE 073076 WITH 100. OBSERVED FIELDS, 5 SLIDES AND 7 FOOD SPECIES

BP-2

DIET SAMPLE 13

FOOD SPEICES

SAKA

SPHA

ECCR

SOLI

SEED

BROM

SALX

FOOD SPECIES

SAKA

SPHA

ECCR

SOLI

SEED

BROM

SALX
RADIATION

SAMPLE NO.

479

DENSITY

1.1087

1.2730

.1054

.0305

.0202

.0101

.0408

REL DENS

REL FREQ

42.1384

45.2830

6.2893

1.8868

1.2579

.6289

2.5157
DIET ANALYSIS FOR CONSUMER ODHE ON DATE 073076 WITH 100. OBSERVED FIELDS, 5 SLIDES AND 5 FOOD SPECIES

$\begin{array}{lcc}\text { BP-2 } & & \text { RADIATION } \\ \text { DIET SAMPLE } & & \begin{array}{c}\text { SAMPLE NO. } \\ 14\end{array} \\ \text { FOOD SPECIES } & \text { FREQUENCY } & \text { DENSITY } \\ \text { SAKA } & 76.2295 & 2.6593 \\ \text { SEED } & 12.0000 & .1278 \\ \text { ECCR } & 13.0000 & .1393 \\ \text { SOLI } & 2.0000 & .0202 \\ \text { MICR } & 2.0000 & .0202 \\ & & \\ \text { FOOD SPECIES } & \text { REL FREQ } & \text { REL DENS } \\ \text { SAKA } & 76.2295 & 89.6351 \\ \text { SEED } & 9.8361 & 4.3089 \\ \text { ECCR } & 10.6557 & 4.6941 \\ \text { SOLI } & 1.6393 & .6810 \\ \text { MICR } & 1.6393 & .6810\end{array}$

DIET ANALYSIS FOR CONSUMER ODHE ON DATE 073075 WITH 100. OBSERVED FIELDS, 5 SLIDES AND 8 FOOD SPECIES

BP.2 DIET SAMPLE 15

$\begin{array}{lc}\text { FOOD SPECIES } & \text { FREQUENCY } \\ \text { SAKA } & 66.0000 \\ \text { SPHA } & 65.0000 \\ \text { ECCR } & 12.0000 \\ \text { POA } & 4.0000 \\ \text { SEED } & 1.0000 \\ \text { SHEP } & 4.0000 \\ \text { BROM } & 1.0000 \\ \text { OPUN } & 1.0000\end{array}$

FOOD SPECIES

SAKA

SPHA

ECCR

POA

SEED

SHEP

BROM

OPUN
RADIATION SAMPLE NO. 476 DENSITY 1.0788 1.0498 .1278 .0408 .0101 .0408 .0101 .0101

REL DENS 45.5528 44.3288 5.3978 1.7237 .4244 1.7237 .4244 .4244
DIET ANALYSIS FOR CONSUMER ODHE ON DATE 073076 WITH 100. OBSERVED FIELDS, 5 SLIDES AND 5 FOOD SPECIES

$\begin{array}{lc}\text { BP-2 } & \\ \text { DIET SAMPLE } & \\ 16 & \\ \text { FOOD SPECIES } & \text { FREQUENCY } \\ \text { SAKA } & 88.0000 \\ \text { SALX } & 22.0000 \\ \text { ECCR } & 5.0000 \\ \text { SEED } & 3.0000 \\ \text { POA } & 10.0000 \\ & \\ \text { FOOD SPECIES } & \text { REL FREQ } \\ \text { SAKA } & 68.7500 \\ \text { SALX } & 17.1875 \\ \text { ECCR } & 3.9063 \\ \text { SEED } & 2.3438 \\ \text { POA } & 7.8125\end{array}$

RADIATION
SAMPLE NO.
477
DENSITY
2.1203
.2485
.0513
.0305
.1054

REL DENS
82.9577
9.7213
2.0069
1.1918
4.1223


DIET ANALYSIS FOR CONSUMER ODHE ON DATE 073076 WITH 100. OBSERVED FIELDS, 5 SLIDES AND 4 FOOD SPECIES

\begin{tabular}{lcc}
\multicolumn{1}{c}{ BP-2 } & & RADIATION \\
DIET SAMPLE & & SAMPLE NO. \\
17 & & 473 \\
FOOD SPECIES & FREQUENCY & DENSITY \\
SAKA & 91.0000 & 2.4079 \\
SOLI & 5.0000 & .0573 \\
ECCR & 3.0000 & .0305 \\
DEPI & 1.0000 & .0101 \\
& & \\
FOOD SPECIES & REL FREQ & REL DENS \\
SAKA & 91.0000 & 96.3275 \\
SOLI & 5.0000 & 2.0519 \\
ECCR & 3.0000 & 1.2785 \\
DEPI & 1.0000 & .4021
\end{tabular}

DIET ANALYSIS FOR CONSUMER ODHE ON DATE 073076 WITH 100. OBSERVED FIELDS, 5 SLIDES AND 4 FOOD SPECIES

$\begin{array}{ccc}\text { BP-2 } & & \text { RADIATION } \\ \text { DIET SAMPLE } & & \begin{array}{c}\text { SAMPLE NO. } \\ 475\end{array} \\ 19 & & \text { DENSITY } \\ \text { FOOD SPECIES } & \text { FREQUENCY } & 4.6052 \\ \text { SPHA } & 99.0000 & .0834 \\ \text { SAKA } & 8.0000 & .0101 \\ \text { BROM } & 1.0000 & .0101 \\ \text { OENO } & 1.0000 & \\ & & \text { REL DENS } \\ \text { FOOD SPECIES } & \text { REL FREQ } & 97.8032 \\ \text { SPHA } & 90.8257 & 1.7708 \\ \text { SAKA } & 7.3394 & .2134 \\ \text { BROM } & .9174 & .2134 \\ \text { OENO } & .9174 & \end{array}$

DIET ANALYSIS FOR CONSUMER ODHE ON DATE 073076 WITH 100. OBSERVED FIELDS, 5 SLIDES AND 5 FOOD SPECIES

$\begin{array}{lc}\text { BP-2 } & \\ \text { DIET SAMPLE } & \\ 18 & \\ \text { FOOD SPECIES } & \text { FREQLENCY } \\ \text { SAKA } & 91.0000 \\ \text { OENO } & 27.0000 \\ \text { POA } & 1.0000 \\ \text { SEED } & 1.0000 \\ \text { ECCR } & 1.0000 \\ & \\ \text { FOOD SPECIES } & \text { REL FREQ } \\ \text { SAKA } & 75.2066 \\ \text { OENO } & 22.3140 \\ \text { POA } & .8264 \\ \text { SEED } & .8264 \\ \text { ECCR } & .8264\end{array}$

RADIATION

SAMPLE NO.

480

DENSITY

2.4079

.3747

.0101

.0101

.0101

REL DENS

87.4724

11.4324

.3651

.3651

.3651

DIET ANIALYSIS FOR CONSUMER ODHE ON DATE 073076 WITH 100. OBSERVED FIELDS, 5 SLIDES AND 7 FOOD SPECIES

$\begin{array}{ccc}\text { BP-2 } & & \text { RADIATION } \\ \text { DIET SAMPLE } & & \text { SAMPLE NO. } \\ 20 & & 472 \\ \text { FOOD SPECIES } & \text { FREQUENCY } & \text { DENSITY } \\ \text { SAKA } & 93.0000 & 1.6094 \\ \text { ASTR } & 1.0000 & .0101 \\ \text { ECCR } & 18.0000 & .7985 \\ \text { SEED } & 3.0000 & .0305 \\ \text { POA } & 3.0000 & .0305 \\ \text { EQUI } & 1.0000 & .0101 \\ \text { SOLI } & 1.0000 & .0701 \\ & & \\ \text { FOOD SPECIES } & R E ! F R E Q & \text { REL DENS } \\ \text { SAK } & 7.7664 & 84.7537 \\ \text { ASTR } & .9346 & .5293 \\ \text { ECCR } & 16.8224 & 10.4505 \\ \text { SEED } & 2.8037 & 1.6040 \\ \text { POA } & 2.8037 & 1.6040 \\ \text { EQUI } & .9346 & .5293 \\ \text { SOLI } & .9346 & .5293\end{array}$


DIET ANALYSIS FOR CONSLMER ODHE ON DATE 073076 WITH 100. OBSERVED FIELDS, 5 SLIDES AND 6 FOOD SPECIES

BP-3 DIET SAMPLE

21

FOOD SPECIES
SAKA
SALX
ECCR
CARE
BROM
PANI

FOOD SPECIES SAKA

SALX

ECCR

CARE

BROM

PANI

RADIATION
SAMPLE NO.
486
DENSITY
1.2379
.2744
.1393
.0408
.0305
.0101

REL DENS
71.4335
15.8368
8.0363
2.3557
1.7577
.5800

REL FREQ

61.2069

20.6897

11.2069

3.4483

2.5862

.8621
DIET ANALYSIS FOR CONSUMER ODHE ON DATE 073076 WITH 100. OBSERVED FIELOS, 5 SLIDES AND 4 FOOD SPECIES

$\begin{array}{lc}\text { BP-3 } & \\ \text { DIET SAMPLE } & \\ 22 & \\ \text { FOOD SPECIES } & \text { FREQUENCY } \\ \text { SPHA } & 82.0000 \\ \text { SAKA } & 22.0000 \\ \text { ECCR } & 11.0000 \\ \text { SALX } & 2.0000 \\ & \\ \text { FOOD SPECIES } & \text { REL FREQ } \\ \text { SPHA } & 70.0855 \\ \text { SAKA } & 18.8034 \\ \text { ECCR } & 9.4017 \\ \text { SALX } & 1.7094 \\ & \\ & \end{array}$

RADIATION
SAMPLE NO.
484
DENSITY
1.7148
.2485
.1165
.0202
REL DENS
81.6572
11.8315
5.5492
.9620

DIET ANALYSIS FOR CONSUMER ODHE ON DATE 073076 WITH 100. OBWERVED FIELDS, 5 SLIDES AND 8 FOOD SPECIES

$\begin{array}{lcc}\text { BP-3 } & & \text { RADIATION } \\ \text { DIET SAMPLE } & & \text { SAMPLE NO. } \\ 23 & & 482 \\ \text { FOOD SPECIES } & \text { FREQUENCY } & \text { DENSITY } \\ \text { SAKA } & 91.0000 & 2.4079 \\ \text { SOLI } & 3.0000 & .0305 \\ \text { SALX } & 21.0000 & .2357 \\ \text { CARE } & 1.0000 & .0101 \\ \text { BROM } & 1.0000 & .0101 \\ \text { LNKG } & 1.0000 & .0101 \\ \text { SEED } & 2.0000 & .0202 \\ \text { ECCR } & 1.0000 & .0101 \\ & & \\ \text { FOOD SPECIES } & \text { REL FREQ } & \text { REL DENS } \\ \text { SAKA } & 75.2066 & 88.0570 \\ \text { SOLI } & 2.4793 & 1.1139 \\ \text { SALX } & 17.3554 & 8.6202 \\ \text { CARE } & .8264 & .3675 \\ \text { BROM } & .8264 & .3675 \\ \text { LNKG } & .8264 & .3675 \\ \text { SEED } & 1.6529 & .7388 \\ \text { ECCR } & .8264 & .3675\end{array}$

DIET ANALYSIS FOR CONSLMER ODHE ON DATE 073076 WITH 100. OBSERVED FIELDS, 5 SLIDES AND 5 FOOD SPECIES

\begin{tabular}{lc}
\multicolumn{2}{c}{ BP-3 } \\
DIET SAMPLE \\
24 & \\
FOOD SPECIES & FREQUENCY \\
SAKA & 96.0000 \\
RAPH & 3.0000 \\
UNKG & 3.0000 \\
SEED & 3.0000 \\
CARE & 1.0000 \\
& \\
FOOD SPECIES & REL FREQ \\
SAKA & 90.5660 \\
RAPH & 2.8302 \\
UNKG & 2.8302 \\
SEED & 2.8302 \\
CARE & .9434
\end{tabular}


DIET ANALYSIS FOR CONSLMER ODHE ON DATE 073076 WITH 100. OBSERVED FIELDS, 5 SLIDES AND 5 FOOD SPECIES

\begin{tabular}{lcc} 
BP-3 & & RADIATION \\
DIET SAMPLE & & SAMPLE NO. \\
\multicolumn{1}{c}{25} & & 488 \\
FOOD SPECIES & FREQUENCY & DENSITY \\
SPHA & 9.0000 & .0943 \\
SAKA & 28.0000 & .3285 \\
SEED & 2.0000 & .0202 \\
SALX & 10.0000 & .1054 \\
ECCR & 5.0000 & .0513 \\
& & \\
FOOD SPECIES & REL FREQ & REL DENS \\
SPHA & 16.6667 & 15.7271 \\
SAKA & 51.8519 & 54.7807 \\
SEED & 3.7037 & 3.3690 \\
SALX & 18.5185 & 17.5697 \\
ECCR & 9.2593 & 8.5536
\end{tabular}

DIET ANALYSIS FOR CONSUMER ODHE ON DATE 073076 WITH 100. OBSERVED FIELDS, 5 SLIDES AND 8 FOOD SPECIES

$\begin{array}{lcc}\text { BP-3 } & & \text { RADIATION } \\ \text { DIET SAMPLE } & & \text { SAMPLE NO. } \\ 27 & & 481 \\ \text { FOOD SPECIES } & \text { FREQUENCY } & \text { DENSITY } \\ \text { SAKA } & 89.0000 & 2.2073 \\ \text { SEED } & 1.0000 & .0101 \\ \text { OENO } & 2.0000 & .0202 \\ \text { ECCR } & 2.0000 & .0202 \\ \text { POA } & 3.0000 & .0305 \\ \text { CARE } & 1.0000 & .0101 \\ \text { EQUI } & 2.0000 & .0202 \\ \text { SOLI } & 1.0000 & .0101 \\ & & \\ \text { FOOD SPECIES } & \text { REL FREQ } & \text { REL DENS } \\ \text { SAKA } & 88.1188 & 94.7941 \\ \text { SEED } & .9901 & .4316 \\ \text { OENO } & 1.9802 & .8676 \\ \text { ECCR } & 1.9802 & .8676 \\ \text { POA } & 2.9703 & 1.3081 \\ \text { CARE } & .9901 & .4316 \\ \text { CQLI } & 1.9802 & .86-6 \\ \text { SOLI } & .9901 & .4316\end{array}$

DIET ANALYSIS FOR CONSUMER ODHE ON DATE 073076 WITH 100. OBSERVED FIELDS, 5 SLIDES AND 6 FOOD SPECIES

$\begin{array}{lcc}\begin{array}{c}\text { BP-3 } \\ \text { DIET SAMPLE }\end{array} & & \begin{array}{c}\text { RADIATION } \\ \text { SAMPLE NO. } \\ 487\end{array} \\ \text { FOOD SPECIES } & \text { FREQUENCY } & \text { DENSITY } \\ \text { SAKA } & 92.0000 & 2.5257 \\ \text { SEED } & 14.0000 & .1508 \\ \text { SALX } & 2.0000 & .0202 \\ \text { ECCR } & 9.0000 & .0953 \\ \text { EQUI } & 1.0000 & .0101 \\ \text { BROM } & 2.0000 & .0202 \\ & & \\ \text { FOOD SPECIES } & \text { REL FREQ } & \text { REL DENS } \\ \text { SAKA } & 76.6667 & 89.5230 \\ \text { SEED } & 11.6667 & 5.3458 \\ \text { SALX } & 1.6667 & .7161 \\ \text { ECCR } & 7.5000 & 3.3428 \\ \text { EQUI } & .8333 & .3562 \\ \text { BROM } & 1.6667 & .7161\end{array}$

DIET ANALYSIS FOR CONSUMER ODHE ON DATE 073076 WITH 100. OBSERVED FIELDS, 5 SLIDES AND 6 FOOD SPECIES

\begin{tabular}{|c|c|c|}
\hline $\begin{array}{c}\text { BP-3 } \\
\text { DIET SAMPLE } \\
28\end{array}$ & & $\begin{array}{c}\text { RADIATION } \\
\text { SAMPLE NO. } \\
490\end{array}$ \\
\hline FOOD SPECIES & FREQUENCY & DENSITY \\
\hline SAKA & 37.0000 & 2.0402 \\
\hline SEED & 10.0000 & .1054 \\
\hline BROM & 7.0000 & .0726 \\
\hline$E C C R$ & 5.0000 & .0513 \\
\hline ASTR & 1.0000 & .0101 \\
\hline SOLI & 3.0000 & .0305 \\
\hline FOOD SPECIES & REL FREQ & REL DENS \\
\hline SAKA & 76.9912 & 88.3230 \\
\hline SEED & 8.8496 & 4.5612 \\
\hline BROM & 6.1947 & 3.1416 \\
\hline ECCR & 4.4248 & 2.2205 \\
\hline ASTR & .8850 & .4351 \\
\hline sOLI & 2.6549 & 1.3186 \\
\hline
\end{tabular}


DIET ANALYSIS FOR CONSUMER ODHE ON DATE 073076 WITH 100. OBSERVED FIELDS, 5 SLIDES AND 8 FOOD SPECIES

$B P-3$

DIET SAMPLE

29

FOOD SPECIES
SALX
SAKA
ECCR
SEED
SOLI
CHNA
SPCR
BROM

FOOD SPECIES

SALX

SAKA

ECCR

SEED

SOLI

CHNA

SPCR

BROM
RADIATION

SAMPLE NO.

483

DENSITY

.0943

2.3026

.0619

.0573

.0101

.0101

.0202

.0101

REL DENS

3.6834

89.9300

2.4166

2.0033

.3925

.3925

.7890

.3925
DIET ANALYSIS FOR CONSUMER ODHE ON DATE 073076 WITH 100. OBSERVED FIELDS, 5 SLIDES, AND 6 FOOD SPECIES

$\begin{array}{lcc}\begin{array}{c}\text { BP-3 } \\ \text { DIET SAMPLE }\end{array} & \text { RADIATION } \\ 30 & & \text { SAMPLE NO. } \\ 485 \\ \text { FOOD SPECIES } & \text { FREQUENCY } & \text { DENSITY } \\ \text { SAKA } & 91.0000 & 2.4079 \\ \text { POA } & 1.0000 & .0101 \\ \text { LUPI } & 1.0000 & .0101 \\ \text { UNKG } & 6.0000 & .0619 \\ \text { UNKS } & 2.0000 & .0202 \\ \text { SEED } & 4.0000 & .0408 \\ & & \\ \text { FOOD SPECIES } & \text { REL FREQ } & \text { REL DENS } \\ \text { SAKA } & 86.6667 & 94.3942 \\ \text { POA } & .9524 & .3940 \\ \text { LUPI } & .9524 & .3940 \\ \text { UNKG } & 5.7143 & 2.4256 \\ \text { UNKS } & 1.9048 & .7920 \\ \text { SEED } & 3.8095 & 1.6 C 03\end{array}$

DIET ANALYSIS FOR CONSUMER ODHE ON DATE 073076 WITH 100. OBSERVED FIELDS, 5 SLIDES AND 4 FOOD SPECIES

BP-4

DIET SAMPLE

31

FOOD SPECIES

SAKA

SEED

RAPH

SOLI

FOOD SPECIES

SAKA

SEED

RAPH

SOLI
RADIATION

SAMPLE NO.

493

DENSITY

.7985

.9943

.0408

.1165

REL DENS

40.9467

50.9843

2.0933

5.9757
DIET ANALYSIS FOR CONSUMER ODHE ON DATE 073076 WITH 100. OBSERVED FIELDS, 5 SLIDES AND 4 FOOD SPECIES

$\begin{array}{cc}\text { BP-4 } & \\ \text { DIET SAMPLE } & \\ 32 & \\ \text { FOOD SPECIES } & \text { FREQUENCY } \\ \text { SAKA } & 87.0000 \\ \text { SALX } & 25.0000 \\ \text { SOLI } & 2.0000 \\ \text { BROM } & 1.0000 \\ & \\ \text { FOOD SPECIES } & \text { REL FREQ } \\ \text { SAKA } & 75.6522 \\ \text { SALX } & 21.7391 \\ \text { SOLI } & 1.7391 \\ \text { BROM } & .8696\end{array}$

RADIATION
SAMPLE NO.
498
DENSITY
2.0402
.2877
.0202
.0101

REL DENS
86.5176
12.1995
.8567
.4262


DIET ANALYSIS FOR CONSUMER ODHE ON DATE 073076 WITH 100. OBSERVED FIELDS, 5 SLIDES AND 3 FOOD SPECIES

$B P-4$

DIET SAMPLE

33

FOOD SPECIES

SAKA

SALX

SOLI

FOOD SPECIES

SAKA

SALX

SOLI
RADIATION

497

FREQUENCY

95.0000

5.0000

4.0000

REL FREQ

91.3462

4.8077

3.8462
DENSITY

2.9957

.0513

.0408

REL DEN

97.0168

1.6611

1.3220
SAMPLE NO.
DIET ANALYSIS FOR CONSUMER ODHE ON DATE 073076 WITH 100. OBSERVED FIELDS, 5 SLIDES AND 6 FOOD SPECIES

$\begin{array}{lcc}\text { BP-4 } & & \text { RADIATION } \\ \text { DIET SAMPLE } & & \text { SAMPLE NO. } \\ 34 & & 499 \\ \text { FOOD SPECIES } & \text { FREQUENCY } & \text { DENSITY } \\ \text { SAKA } & 94.0000 & 2.8134 \\ \text { SALX } & 8.0000 & .0834 \\ \text { ECCR } & 6.0000 & .0619 \\ \text { CARE } & 1.0000 & .0101 \\ \text { SPHA } & 1.0000 & .0101 \\ \text { BROM } & 1.0000 & .0101 \\ & & \\ \text { FOOD SPECIES } & \text { REL FREQ } & \text { REL DENS } \\ \text { SAKA } & 84.6847 & 94.1312 \\ \text { SALX } & 7.2072 & 2.7898 \\ \text { ECCR } & 5.4054 & 2.0702 \\ \text { CARE } & .9009 & .3363 \\ \text { SPHA } & .9009 & .3363 \\ \text { BROM } & .9009 & .3363\end{array}$

DIET ANALYSIS FOR CONSUMER ODHE ON DATE 073076 WITH 100. OBSERVED FIELDS, 5 SLIDES AND 4 FOOD SPECIES

$\begin{array}{lcc}\text { BP-4 } & & \text { RADIATION } \\ \text { DIET SAMPLE } & & \text { SAMPLE NO. } \\ 35 & & 496 \\ \text { FOOD SPECIES } & \text { FREQUENCY } & \text { DENSITY } \\ \text { SAKA } & 96.0000 & 3.2189 \\ \text { SEED } & 6.0000 & .0619 \\ \text { SALX } & 4.0000 & .0408 \\ \text { ECCR } & 2.0000 & .0202 \\ & & \\ \text { FOOD SPECIES } & \text { REL FREQ } & \text { REL DENS } \\ \text { SAKA } & 88.8889 & 96.3223 \\ \text { SEED } & 5.5556 & 1.8516 \\ \text { SALX } & 3.7037 & 1.2216 \\ \text { ECCR } & 1.8519 & .6046\end{array}$

DIET ANALYSIS FOR CONSUMER ODHE ON DATE 073076 WITH 100. OBSERVED FIELDS, 5 SLIDES AND 6 FOOD SPECIES

$\begin{array}{lc}\text { BP-4 } & \\ \text { DIET SAMPLE } & \\ 36 & \\ \text { FOOD SPECIES } & \text { FREQUENCY } \\ \text { SAKA } & 94.0000 \\ \text { ECCR } & 7.0000 \\ \text { SOLI } & 1.0000 \\ \text { UNKS } & 2.0000 \\ \text { SEED } & 3.0000 \\ \text { BROM } & 2.0000 \\ & \\ \text { FOOD SPECIES } & \text { REL } 8 R E Q \\ \text { SAKA } & 86.2385 \\ \text { ECCR } & 6.4220 \\ \text { SOLI } & .9174 \\ \text { UNKS } & 1.8349 \\ \text { SEED } & 2.7523 \\ \text { BROM } & 1.8349\end{array}$


DIET ANALYSIS FOR CONSUMER ODHE ON DATE 073076 WITH 100. OBSERVED FIELDS, 5 SLIDES AND 7 FOOD SPECIES

$\begin{array}{lcc}\begin{array}{c}\text { BP.4 } \\ \text { DIET SAMPLE }\end{array} & & \begin{array}{c}\text { RADIATION } \\ \text { SAMPLE NO. }\end{array} \\ \text { SAKA } & & 495 \\ \text { SALX } & 87.0000 & 2.0402 \\ \text { BROM } & 8.0000 & .0834 \\ \text { SOLI } & 3.0000 & .0305 \\ \text { ECCR } & 2.0000 & .0202 \\ \text { OENO } & 3.0000 & .0305 \\ \text { SEED } & 1.0000 & .0101 \\ & 1.0000 & .0101 \\ \text { FOOD SPECIES } & \text { REL FREQ } & \\ \text { SAKA } & 82.8571 & \text { REL DENS } \\ \text { SALX } & 7.6190 & 91.7026 \\ \text { BROM } & 2.8571 & 3.7478 \\ \text { SOLI } & 1.9048 & 1.3691 \\ \text { ECCR } & 2.8571 & .9081 \\ \text { OENO } & .9524 & 1.3691 \\ \text { SEED } & .9524 & .4517 \\ \end{array}$

DIET ANALYYSIS FOR CONSUMER ODHE ON DATE 073076 WITH 100. OBSERVED FIELDS, 5 SLIDES AND 4 FOOD SPECIES

$\begin{array}{lcc}\text { BF }-4 & & \text { RADIATION } \\ \text { DIET SAMPLE } & & \text { SAMPLE NO. } \\ 38 & & 500 \\ \text { FOOD SPECIES } & \text { FREQUENCY } & \text { DENSITY } \\ \text { SALX } & 100.0000 & .2485 \\ \text { SAKA } & 22.0000 & .0101 \\ \text { CARE } & 1.0000 & .0202 \\ \text { BROM } & 2.0000 & \\ \text { FOOD SPECIES } & \text { REL FREQ } & \text { REL DENS } \\ \text { SALX } & 80.0000 & * \cdots * * * * * * \\ \text { SAKA } & 17.6000 & 89.1455 \\ \text { CARE } & .8000 & 3.6060 \\ \text { BROM } & 1.6000 & 7.2485\end{array}$

DIET ANALYSIS FOR CONSUMER ODHE ON DATE 073076 WITH 100. OBSERVED FIELDS, 5 SLIDES AND 4 FOOD SPECIES

$\begin{array}{ccc}\text { BP-4 } & & \text { RADIATION } \\ \text { DIET SAMPLE } & & \text { SAMPLE NO. } \\ 39 & & 494 \\ \text { FOOD SPECIES } & \text { FREQUENCY } & \text { DENSITY } \\ \text { SAKA } & 74.0000 & 1.3471 \\ \text { ECCR } & 35.0000 & .4308 \\ \text { SALX } & 6.0000 & .0619 \\ \text { BROM } & 3.0000 & .0305 \\ & & \\ \text { FOOD SPECIES } & \text { REL FREQ } & \text { REL DENS } \\ \text { SAKA } & 62.7119 & 72.0287 \\ \text { ECCR } & 29.6610 & 23.0342 \\ \text { SALX } & 5.0847 & 3.3085 \\ \text { BROM } & 2.5424 & 1.6287\end{array}$

DIET ANALYSIS FOR CONSUMER ODHE ON DATE 073076 WITH 100. OBSERVED FIELDS, 5 SLIDES AND 2 FOOD SPECIES

$\begin{array}{lcc}\text { BP-4 } & & \text { RADIATION } \\ \text { DIET SAMPLE } & & \text { SAMPLE NO. } \\ 40 & & 491 \\ & & \\ \text { FOOD SPECIES } & \text { FREQUENCY } & \text { DENSITY } \\ \text { SAKA } & 98.0000 & 3.9120 \\ \text { SEED } & 2.0000 & .0202 \\ & & \\ \text { FOOD SPECIES } & \text { REL FREQ } & \text { REL DENS } \\ \text { SAKA } & 98.0000 & 99.4862 \\ \text { SEED } & 2.0000 & .5138\end{array}$


DIET ANALYSIS FOR CONSUMER ODHE ON DATE 072976 WITH 100. OBSERVED FIELDS, 5 SLIDES AND 5 FOOD SPECIES

$\begin{array}{llc}\begin{array}{c}\text { CM-1 } \\ \text { DIET SAMPLE }\end{array} & \text { RADIATION } \\ 41 & & \text { SAMPLE NO. } \\ \text { SO2 } & \text { DENSITY } \\ \text { FOOD SPECIES } & \text { FREQUENCY } & .9943 \\ \text { SALX } & 63.0000 & .2357 \\ \text { MELI } & 21.0000 & .1165 \\ \text { BROM } & 11.0000 & .0619 \\ \text { SOLI } & 6.0000 & .0513 \\ \text { SAKA } & 5.0000 & \\ & & \text { REL DENS } \\ \text { FOOD SPECIES } & \text { REL FREQ } & 68.1145 \\ \text { SALX } & 59.4340 & 16.1489 \\ \text { MELI } & 19.8113 & 7.9835 \\ \text { BROM } & 10.3774 & 4.2390 \\ \text { SOLI } & 5.6604 & 3.5140 \\ \text { SAKA } & 4.7170 & \end{array}$

DIET ANALYSIS FOR CONSUMER ODHE ON DATE 072976 WITH 100. OBSERVED FIELDS, 5 SLIDES AND 8 FOOD SPECIES

$\begin{array}{lcc}\begin{array}{c}\text { GM-1 } \\ \text { DIET SAMPLE }\end{array} & \begin{array}{c}\text { RADIATION } \\ \text { SAMPLE NO. }\end{array} \\ \text { FOOD SPECIES } & \text { FREQUENCY } & \text { DENSITY } \\ \text { SOLI } & 87.0000 & 2.0402 \\ \text { UNKF } & 12.0000 & .1278 \\ \text { SAKA } & 4.0000 & .0408 \\ \text { LACT } & 2.0000 & .0202 \\ \text { BROM } & 2.0000 & .0202 \\ \text { EQUI } & 2.0000 & .0202 \\ \text { MELI } & 2.0000 & .0202 \\ \text { SPHA } & 1.0000 & .0101 \\ & & \\ \text { FOOD SPECIES } & \text { REL FREQ } & \text { REL DENS } \\ \text { SOLI } & 77.6786 & 88.7154 \\ \text { LNKF } & 10.7143 & 5.5586 \\ \text { SAKA } & 3.5714 & 1.7751 \\ \text { LACT } & 1.7857 & .8785 \\ \text { BROM } & 1.7857 & .8785 \\ \text { EQUI } & 1.7857 & .8785 \\ \text { MELI } & 1.7857 & .8785 \\ \text { SPHA } & .8929 & .4370\end{array}$

DIET ANALYSIS FOR CONSUMER ODHE ON DATE 072976 WITH 100. OBSERVED FIELDS, 5 SLIDES AND 5 FOOD SPECIES

$\begin{array}{lcc}\text { CM-1 } & & \text { RADIATION } \\ \text { DIET SAMPLE } & & \begin{array}{c}\text { SAMPLE NO. } \\ 42\end{array} \\ \text { FOOD SPECIES } & \text { FREQUENCY } & \text { DENSITY } \\ \text { SOLI } & 88.0000 & 2.1203 \\ \text { MELI } & 16.0000 & .1744 \\ \text { LACT } & 3.0000 & .0305 \\ \text { RAPH } & 2.0000 & .0202 \\ \text { BAHY } & 2.0000 & .0202 \\ & & \\ \text { FOOD SPECIES } & \text { REL FREQ } & \text { REL DENS } \\ \text { SOLI } & 79.2793 & 89.6335 \\ \text { MELI } & 14.4144 & 7.3707 \\ \text { LACT } & 2.7027 & 1.2877 \\ \text { RAPH } & 1.8018 & .8541 \\ \text { BAHY } & 1.8018 & .8541\end{array}$

DIET ANALYSIS FOR CONSUMER ODHE ON DATE 072976 WITH 100. OBSERVED FIELDS, 5 SLIDES AND 3 FOOD SPECIES

$\begin{array}{cc}\text { CM-1 } & \\ \text { DIET SAMPLE } & \\ 44 & \\ \text { FOOD SPECIES } & \text { FREQUSNCY } \\ \text { SOLI } & 99.0000 \\ \text { MELI } & 11.0000 \\ \text { UNKF } & 2.0000 \\ & \\ \text { FOOD SPECIES } & \text { REL FREQ } \\ \text { SOLI } & 88.3929 \\ \text { MELI } & 9.8214 \\ \text { UNKF } & 1.7857\end{array}$


DIET ANALYSIS FOR CONSUMER ODHE ON DATE 072976 WITH 100. OBSERVED FIELDS, 5 SLIDES AND 5 FOOD SPECIES

\begin{tabular}{|c|c|c|}
\hline $\begin{array}{c}\text { GM-1 } \\
\text { DIET SAMPLE } \\
45\end{array}$ & & $\begin{array}{c}\text { RADIATION } \\
\text { SAMPLE NO. } \\
405\end{array}$ \\
\hline $\begin{array}{l}\text { FOOD SPECIES } \\
\text { SALX }\end{array}$ & $\begin{array}{l}\text { FREQUENCY } \\
59.0000\end{array}$ & $\begin{array}{l}\text { DENSITY } \\
.8916\end{array}$ \\
\hline BAHY & 49.0000 & .6733 \\
\hline MELI & 22.0000 & .2485 \\
\hline SOLI & 5.0000 & .0513 \\
\hline MUHL & 1.0000 & .0101 \\
\hline FOOD SPECIES & REL FREQ & REL DENS \\
\hline SALX & 43.3824 & 47.5583 \\
\hline BAHY & 36.0294 & 35.9165 \\
\hline MELI & 16.1765 & 13.2531 \\
\hline SOLI & 3.6765 & 2.7360 \\
\hline MUHL & .7353 & .5361 \\
\hline
\end{tabular}

DIET ANALYSIS FOR CONSUMER ODHE ON DATE 073076 WITH 120. OBSERVED FIELDS, 6 SLIDES AND 11 FOOD SPECIES

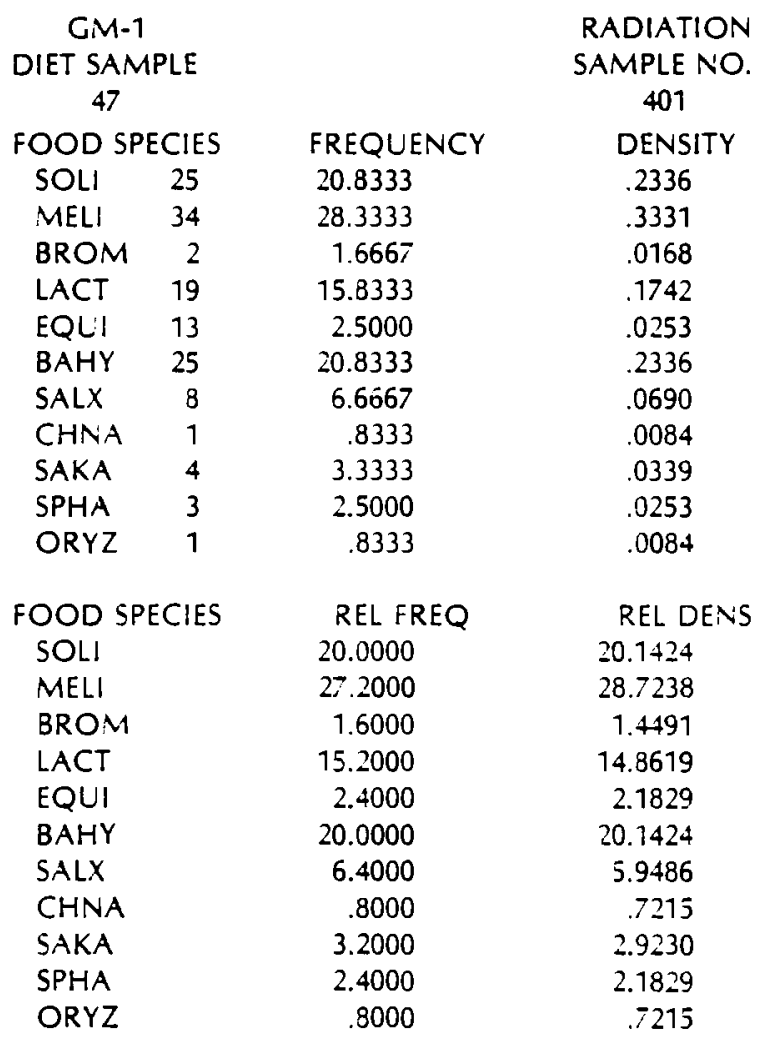

DIET ANALYSIS FOR CONSUMER ODHE ON DATE 072976 WITH 100. OBSERVED FIELDS, 5 SLIDES AND 5 FOOD SPECIES

$\begin{array}{lcc}\text { GM-1 } & & \text { RADIATION } \\ \text { DIET SAMPLE } & & \text { SAMPLE NO } \\ 46 & & 407 \\ \text { FOOD SPECIES } & \text { FREQUENCY } & \text { DENSITY } \\ \text { SOLI } & 90.0000 & 2.3026 \\ \text { MELI } & 9.0000 & .0943 \\ \text { EQUI } & 9.0000 & .0943 \\ \text { LACT } & 11.0000 & .1165 \\ \text { ORHY } & 1.0000 & .0101 \\ & & \\ \text { FOOD SPECIES } & \text { REL FREQ } & \text { REL DENS } \\ \text { SOLI } & 75.0000 & 87.9591 \\ \text { MELI } & 7.5000 & 3.6027 \\ \text { EQUI } & 7.5000 & 3.6027 \\ \text { LACT } & 9.1667 & 4.4516 \\ \text { ORHY } & .8333 & .3839\end{array}$

DIET ANALYSIS FOR CONSUMER ODHE ON DATE 072976 WITH 100. OBSERVED FIELDS, 5 SLIDES AND 8 FOOD SPECIES

$\begin{array}{lcc}\text { GM-1 } & & \text { RADIATION } \\ \text { DIET SAMPLE } & & \text { SAMPLE NO. } \\ 48 & & 409 \\ \text { FOOD SPECIES } & \text { FREQUENCY } & \text { DENSITY } \\ \text { MELI } & 38.0000 & .4780 \\ \text { LACT } & 20.0000 & .2231 \\ \text { SOLI } & 56.0000 & .8210 \\ \text { EQUI } & 7.0000 & .0726 \\ \text { BROM } & 2.0000 & .0202 \\ \text { SALX } & 3.0000 & .0305 \\ \text { CARE } & 5.0000 & .0513 \\ \text { ORHY } & 1.0000 & .0101 \\ & & \\ \text { FOD SPECIES } & R E L F R E Q & \text { REL DENS } \\ \text { MELI } & 28.7879 & 28.0088 \\ \text { LACT } & 15.1515 & 13.07+3 \\ \text { SOLI } & 42.4242 & 48.1024 \\ \text { EQUI } & 5.3030 & 4.2520 \\ \text { BROM } & 1.5152 & 1.1837 \\ \text { SALX } & 2.2727 & 1.7846 \\ \text { C.ARE } & 3.7879 & 3.0052 \\ \text { ORHY } & .7576 & .5889\end{array}$


DIET ANALYSIS FOR CONSUMER ODHE ON DATE 072976 WITH 100. OBSERVED FIELD, 5 SILIDES AND 4 FOOD SPECIES

GM-1 DIET SAMPLE 49 FOOD SPECIES

SALX

MELI

UNKS

SAKA

FOOD SPECIES

SALX

MELI

UNKS

SAKA
RADIATION

SAMPLE NO.

406

DENSITY

FREQUENCY

60.0000

46.0000

1.0000

2.0000

REL FREQ

55.0459

42.2018

.9174

1.8349
.9163

.6762

.0101

.0202

REL DENS

58.6340

39.4301

.6431

1.2928
DIET ANALYSIS FOR CONSUMER ODHE ON DATE 072976 WITH 100. OBSERVED FIELDS, 5 SLIDES AND 7 FOOD SPECIES

$\begin{array}{lcc}\begin{array}{c}\text { GM-1 } \\ \text { DIET SAMPLE }\end{array} & & \text { RADIATION } \\ 50 & & \text { SAMPLE NO. } \\ 408 \\ \text { FOOD SPECIES } & \text { FREQUENCY } & \text { DENSITY } \\ \text { MELI } & 77.0000 & 1.4697 \\ \text { SALX } & 15.0000 & .1625 \\ \text { BAHY } & 5.0000 & .0513 \\ \text { SAKA } & 3.0000 & .0305 \\ \text { SOLI } & 3.0000 & .0305 \\ \text { OENO } & 1.0000 & .0101 \\ \text { SEED } & 1.0000 & .0101 \\ & & \\ \text { FOOD SPECIES } & \text { REL FREQ } & \text { REL DENS } \\ \text { MELI } & 73.3333 & 83.2910 \\ \text { SALX } & 14.2857 & 9.2104 \\ \text { BAHY } & 4.7619 & 2.9069 \\ \text { SAKA } & 2.8571 & 1.7262 \\ \text { SOLI } & 2.8571 & 1.7262 \\ \text { OENO } & .9524 & .5696 \\ \text { SEED } & .9524 & .5696\end{array}$

DIET ANALYSIS FOR CONSUMER ODHE ON DATE 072976 WITH 100. OBSERVED FIELDS, 5 SLIDES AND 8 FOOD SPECIES WITH 100. OBSERVED FIELDS, 5 SLIDES AND 2 FOOD SPECIES

$G M-2$

DIET SAMPLE

51

FOOD SPECIES

SOLI

BROM

FREQUENCY

100.0000

1.0000

RADIATION

SAMPLE NO.

412

DENSITY

.0101

FOOD SPECIES

SOLI

BROM
GM-2

DIET SAMPLE

52

FOOD SPECIES

SOLI

SAKA

SEED

AGRO

BROM

MELI

BAHY

LACT

FOOD SPECIES

SOLI

SAKA

SEED

ACRO

BROM

MELI

BAHY

LACT
RADIATION

SAMPLE NO.

415

DENSITY

1.2730

.1393

.1863

.0202

.0408

.0408

.0202

.0101

REL DENS

73.5539

8.0468

10.7664

1.1673

2.3588

2.3588

1.1673

.5807 
DIET ANALYSIS FOR CONSUMER ODHE ON DATE 072976 WITH 100. OBSERVED FIELDS, 5 SLIDES AND 11 FOOD SPECIES

GM-2

DIET SAMPLE

53

FOOD SPECIES

MELI

SOLI

ANDR

BAHY

UNKS

LACT

BROM

SALX

SEED

POA

LESQ

FOOD SPECIES

MELI

SOI.I

ANDR

BAHY

UNKS

LACT

BROM

SALX

SEED

POA

LESQ
RADIATION

SAMPLE NO.

420

DENSITY

.6162

.6162

.2614

.0305

.0834

.0101

.0202

.1863

.0101

.0101

.0101

REL DENS

REL FREQ

30.8725

30.8725

15.4362

2.0134

5.3691

.6711

1.3423

11.4094

.6711

.6711

.6711
DIET ANALYSIS FOR CONSUMER ODHE ON DATE 072976 WITH 100. OBSERVED FIELDS, 5 SLIDES AND 7 FOOD SPECIES

$\begin{array}{lcc}\text { GM-2 } & & \text { RADIATION } \\ \text { DIET SAMPLE } & & \begin{array}{c}\text { SAMLE NO. } \\ 54\end{array} \\ \text { FOOD SPECIES } & \text { FREQUENCY } & \text { OENSITY } \\ \text { SOLI } & 33.0000 & .4005 \\ \text { MELI } & 44.0000 & .5798 \\ \text { SALX } & 44.0000 & .5798 \\ \text { LACT } & 5.0000 & .0513 \\ \text { SPHA } & 7.0000 & .0726 \\ \text { BROM } & 1.0000 & .0101 \\ \text { CARE } & 1.0000 & .0101 \\ & & \\ \text { FOOD SPECIES } & \text { REL FREQ } & \text { REL DENS } \\ \text { SOLI } & 24.4444 & 23.5011 \\ \text { MELI } & 32.5926 & 34.0253 \\ \text { SALX } & 32.5926 & 34.0253 \\ \text { LACT } & 3.7037 & 3.0100 \\ \text { SPHA } & 5.1852 & 4.2586 \\ \text { BROM } & .7407 & .5898 \\ \text { CARE } & .7407 & .5898\end{array}$

DIET ANALYSIS FOR CONSUMER ODHE ON DATE 072976 WITH 100. OBSERVED FIELDS, 5 SLIDES AND 5 FOOD SPECIES

$\begin{array}{lcc}\begin{array}{c}\text { CM-2 } \\ \text { DIET SAMPLE }\end{array} & \begin{array}{c}\text { RADIATION } \\ \text { SAMPLE NO. } \\ \text { 55 }\end{array} & 413 \\ \text { FOOD SPECIES } & \text { FREQUENCY } & \text { DENSITY } \\ \text { MELI } & 96.0000 & 3.2189 \\ \text { SALX } & 83.0000 & 1.7720 \\ \text { CARE } & 1.0000 & .0101 \\ \text { BROM } & 1.0000 & .0101 \\ \text { SAKA } & 1.0000 & .0101 \\ & & \\ \text { FOOD SPECIES } & \text { REL FREQ } & \text { REL DENS } \\ \text { MELI } & 52.7473 & 64.1085 \\ \text { SALX } & 45.6044 & 35.2910 \\ \text { CARE } & .5495 & .2002 \\ \text { BROM } & .5495 & .2002 \\ \text { SAKA } & .5495 & .2002\end{array}$

DIET ANALYSIS FOR CONSUMER ODHE ON DATE 072976 WITH 100. OBSERVED FIELDS, 5 SLIDES AND 6 FOOD SPECIES

$\begin{array}{lcc}\begin{array}{c}\text { GM-2 } \\ \text { DIET SAMPLE }\end{array} & & \text { RADIATION } \\ 56 & & \text { SAMPLE NO. } \\ \text { FOOD SPECIES } & \text { FREQUENCY } & 411 \\ \text { DENSITY } \\ \text { MELI } & 94.0000 & 2.8134 \\ \text { SALX } & 65.0000 & 1.0498 \\ \text { PHLO } & 8.0000 & .0834 \\ \text { UNKF } & 1.0000 & .0101 \\ \text { TYPH } & 2.0000 & .0202 \\ \text { BROM } & 1.0000 & .0101 \\ & & \\ \text { FOOD SPECIES } & \text { REL FREQ } & \text { REL DENS } \\ \text { MELI } & 54.9708 & 70.5661 \\ \text { SALX } & 38.0117 & 26.3317 \\ \text { PHLO } & 4.6734 & 2.091 .4 \\ \text { UNKF } & .5848 & .2521 \\ \text { TYPH } & 1.1696 & .5067 \\ \text { BROM } & .5848 & .2521\end{array}$


DIET ANALYSIS FOR CONSUMER ODHE ON DATE 072976 WITH 100. OBSERVED FIELDS, 5 SLIDES AND 6 FOOD SPECIES

$\begin{array}{ccc}\text { GM-2 } & & \text { RADIATION } \\ \text { DIET SAMPLE } & & \text { SAMPLE NO. } \\ 57 & & 417 \\ \text { FOOD SPECIES } & \text { FREQUENCY } & \text { DENSITY } \\ \text { SALX } & 50.0000 & .6931 \\ \text { SOLI } & 22.0000 & .2485 \\ \text { ASTR } & 66.0000 & 1.0788 \\ \text { LACT } & 2.0000 & .0202 \\ \text { SEED } & 9.0000 & .0943 \\ \text { MELI } & 2.0000 & .0202 \\ & & \\ \text { FOOD SPECIES } & R E L \mathrm{FREQ} & \text { REL DENS } \\ \text { SALX } & 33.1126 & 32.1626 \\ \text { SOLI } & 14.5695 & 11.5288 \\ \text { ASTR } & 43.7086 & 50.0577 \\ \text { LACT } & 1.3245 & .9374 \\ \text { SEED } & 5.9603 & 4.3761 \\ \text { MELI } & 1.3245 & .9374\end{array}$

DIET ANALYSIS FOR CONSUMER ODHE ON DATE 072976 WITH 100. OBSERVED FIELDS, 5 SLIDES AND 6 FOOD SPECIES

$\begin{array}{lcc}\text { GM-2 } & & \text { RADIATION } \\ \text { DIET SAMPLE } & & \text { SAMPLE NO. } \\ 59 & & 416 \\ \text { FOOD SPECIES } & \text { FREQUENCY } & \text { DENSITY } \\ \text { PUTR } & 86.0000 & 1.9661 \\ \text { SEED } & 7.0000 & .0726 \\ \text { MELI } & 4.0000 & .0408 \\ \text { SOLI } & 2.0000 & .0202 \\ \text { LACT } & 1.0000 & .0101 \\ \text { UNKF } & 1.0000 & .0101 \\ & & \\ \text { FOOD SPECIES } & \text { REL FREQ } & \text { REL DENS } \\ \text { PUTR } & 85.1485 & 92.7495 \\ \text { SEED } & 6.9307 & 3.4235 \\ \text { MEL1 } & 3.9604 & 1.9257 \\ \text { SOLI } & 1.9802 & .9530 \\ \text { LACT } & .9901 & .4741 \\ \text { UNKF } & .9901 & .4741\end{array}$

DIET ANALYSIS FOR CONSUMER ODHE ON DATE 072976 WITH 100. OBSERVED FIELDS, 5 SLIDES AND 7 FOOD SPECIES

$\begin{array}{ccc}\text { GM-2 } & & \text { RADIATION } \\ \text { DIET SAMPLE } & & \text { SAMPLE NO. } \\ 58 & & 419 \\ \text { FOOD SPECIES } & \text { FREQUENCY } & \text { DENSITY } \\ \text { MELI } & 13.0000 & .1393 \\ \text { SOLI } & 69.0000 & 1.1712 \\ \text { SALX } & 14.0000 & .1508 \\ \text { LACT } & 3.0000 & .0305 \\ \text { SPHA } & 3.0000 & .0305 \\ \text { OENO } & 1.0000 & .0101 \\ \text { ARIS } & 1.0000 & .0101 \\ & & \\ \text { FOOD SPECIES } & \text { REL FREQ } & \text { REL DENS } \\ \text { MELI } & 12.5000 & 9.0296 \\ \text { SOLI } & 66.3462 & 75.9381 \\ \text { SALX } & 13.4615 & 9.7792 \\ \text { LACT } & 2.8846 & 1.9749 \\ \text { SPHA } & 2.8846 & 1.9749 \\ \text { OENO } & .9615 & .6517 \\ \text { ARIS } & .9615 & .6517\end{array}$

DIET ANALYSIS FOR CONSUMER ODHE ON DATE 072976 WITH 100. OBSERVED FIELDS, 5 SLIDES AND 8 FOOD SPECIES

\begin{tabular}{|c|c|c|}
\hline $\begin{array}{c}\text { GM-2 } \\
\text { DIET SAMPLE } \\
60\end{array}$ & & $\begin{array}{c}\text { RADIATION } \\
\text { SAMPLE NO. } \\
414\end{array}$ \\
\hline FOOD SPECIES & FREQUENCY & DENSITY \\
\hline MELI & 21.0000 & .2357 \\
\hline SALX & 13.0000 & .1393 \\
\hline SOLI & 41.0000 & .5276 \\
\hline SEED & 7.0000 & .0726 \\
\hline SPHA & 1.0000 & .0101 \\
\hline $\mathrm{LACT}$ & 3.0000 & .0305 \\
\hline BAHY & 1.0000 & .0707 \\
\hline EQUI & 1.0000 & .0101 \\
\hline FOOD SPECIES & REL FREQ & REL DENS \\
\hline MELI & 23.8636 & 22.7576 \\
\hline SALX & 14.7727 & 13.4449 \\
\hline SOLI & 46.5909 & 50.9397 \\
\hline SEED & 7.9545 & 7.0063 \\
\hline SPHA & 1.1364 & .9703 \\
\hline LACT & 3.4091 & 2.9407 \\
\hline BAHY & 1.1364 & .9703 \\
\hline EQL'I & 1.1364 & .9703 \\
\hline
\end{tabular}


DIET ANALYSIS FOR CONSUMER ODHE ON DATE 073076 WITH 100. OBSERVED FIELDS, 5 SLIDES AND 7 FOOD SPECIES

$$
\begin{gathered}
\text { GM-3 } \\
\text { DIET SAMPLE }
\end{gathered}
$$

61

FOOD SPECIES

SOLI

SALX

MELI

BROM

BAHY

EQUI

SAKA

FOOD SPECIES

SOLI

SALX

MELI

BROM

BAHY

EQUI

SAKA
RADIATION

SAMPLE NO.

430

DENSITY

.3567

.5276

.6539

.0513

.2357

.0101

.0202

REL DENS

19.2225

28.4361

35.2425

2.7644

12.7040

.5417

1.0888
DIET ANALYSIS FOR CONSUMER ODHE ON DATE 073076 WITH 100. OBSERVED FIELDS, 5 SLIDES AND 7 FOOD

\begin{tabular}{|c|c|c|}
\hline $\begin{array}{c}\text { CMM-3 } \\
\text { DIET SAMPLE } \\
62\end{array}$ & & $\begin{array}{c}\text { RADIATION } \\
\text { SAMPLE NO. } \\
424\end{array}$ \\
\hline FOOD SPECIES & FREQUENCY & DENSITY \\
\hline MELI & 45.0000 & .5978 \\
\hline SALX & 15.0000 & .1625 \\
\hline SOLI & 17.0000 & .1863 \\
\hline BAHY & 30.0000 & .3567 \\
\hline SAKA & 5.0000 & .0513 \\
\hline BROM & 3.0000 & .0305 \\
\hline LACT & 1.0000 & .0101 \\
\hline FOOD SPECIES & REL FREQ & REL DENS \\
\hline MELI & 38.7931 & 42.8507 \\
\hline SALX & 12.9310 & 11.6487 \\
\hline SOLI & 14.6552 & 13.3554 \\
\hline BAHY & 25.8621 & 25.5651 \\
\hline SAKA & 4.3103 & 3.6765 \\
\hline BROM & 2.5862 & 2.1832 \\
\hline LACT & .8621 & .7204 \\
\hline
\end{tabular}
SPECIES
DIET ANALYSIS FOR CONSUMER ODHE ON DATE 073076 WITH 100. OBSERVED FIELDS, 5 SLIDES AND 5 FOOD SPECIES

$\begin{array}{lcc}\text { GM-3 } & & \text { RADIATION } \\ \text { DIET SAMPLE } & & \begin{array}{c}\text { SAMPLE NO. } \\ 63\end{array} \\ \text { FO28 } \\ \text { FOOD SPECIES } & \text { FREQUENCY } & \text { DENSITY } \\ \text { SOLI } & 29.0000 & .3425 \\ \text { MELI } & 9.0000 & .0943 \\ \text { SAKA } & 70.0000 & 1.2040 \\ \text { SALX } & 5.0000 & .0513 \\ \text { BAHY } & 2.0000 & .0202 \\ & & \\ \text { FOOD SPECIES } & \text { REL FREQ } & \text { REL DENS } \\ \text { SOLI } & 25.2174 & 20.0021 \\ \text { MELI } & 7.8261 & 5.5079 \\ \text { SAKA } & 60.8696 & 70.3144 \\ \text { SALX } & 4.3478 & 2.9956 \\ \text { BAHY } & 1.7391 & 1.1799\end{array}$


DIET ANALYSIS FOR CONSUMER ODHE ON DATE 073076 WITH 100. OBSERVED FIELDS, 5 SLIDES AND 7 FOOD SPECIES

$\begin{array}{lcc}\begin{array}{c}\text { GIM-3 } \\ \text { DIET SAMPLE }\end{array} & & \begin{array}{c}\text { RADIATION } \\ \text { SAMPLE NO. } \\ 425\end{array} \\ \text { FOOD SPECIES } & \text { FREQUENCY } & \text { DENSITY } \\ \text { SOLI } & 88.0000 & 2.1203 \\ \text { BROM } & 5.0000 & .0513 \\ \text { MELI } & 2.0000 & .0202 \\ \text { PIPU } & 6.0000 & .0619 \\ \text { MOSS } & 1.0000 & .0101 \\ \text { SAKA } & 1.0000 & .0101 \\ \text { LACT } & 1.0000 & .0101 \\ & & \\ \text { FOOD SPECIES } & \text { REL FREQ } & \text { REL DENS } \\ \text { SOLI } & 84.6154 & 92.8399 \\ \text { BROM } & 4.8077 & 2.2460 \\ \text { MELI } & 1.9231 & .8846 \\ \text { PIPL } & 5.7692 & 2.7093 \\ \text { MOSS } & .9615 & .4401 \\ \text { SAKA } & .9615 & .4401 \\ \text { LACT } & .9615 & .4401\end{array}$

DIET ANALYSIS FOR CONSUMER ODHE ON DATE 073076 WITH 100. OBSERVED FIELDS, 5 SLIDES AND 6 FOOD SPECIES

\begin{tabular}{|c|c|c|}
\hline $\begin{array}{c}\text { GM-3 } \\
\text { DIET SAMPLE } \\
67\end{array}$ & & $\begin{array}{c}\text { RADIATION } \\
\text { SAMPLE NO. } \\
426\end{array}$ \\
\hline FOOD SPECIES & FREQUENCY & DENSITY \\
\hline MELI & 15.0000 & .1625 \\
\hline SOLI & 71.0000 & 1.2379 \\
\hline SAKA & 8.0000 & .0834 \\
\hline SEED & 3.0000 & .0305 \\
\hline BROM & 6.0000 & .0619 \\
\hline ARTE & 1.0000 & .0101 \\
\hline FOOD SPECIES & REL FREQ & REL DENS \\
\hline MELI & 14.4231 & 10.2461 \\
\hline SOLI & 68.2692 & 78.0422 \\
\hline SAKA & 7.6923 & 5.2568 \\
\hline SEED & 2.8846 & 1.9203 \\
\hline BROM & 5.7692 & 3.9010 \\
\hline ARTE & .9615 & .6336 \\
\hline
\end{tabular}

DIET ANALYSIS FOR CONSUMER ODHE ON DATE 073076 WITH 100. OBSERVED FIELDS, 5 SLIDES AND 4 FOOD SPECIES

$\begin{array}{lcc}\begin{array}{c}\text { GM-3 } \\ \text { DIET SAMPLE }\end{array} & \text { RADIATION J. } \\ \text { SAMPLE NO. } \\ \text { FOOD SPECIES } & \text { FREQUENCY } & 423 \\ \text { SOLI } & 97.0000 & \text { DENSITY } \\ \text { MELI } & 5.0000 & 3.5066 \\ \text { BROM } & 1.0000 & .0513 \\ \text { ARTE } & 1.0000 & .0101 \\ & & .0101 \\ \text { FOOD SPECIES } & \text { REL FREQ } & \\ \text { SOL! } & 93.2692 & \text { REL DENS } \\ \text { MELI } & 4.8077 & 98.0046 \\ \text { BROM } & .9615 & 1.4336 \\ \text { ARTE } & .9615 & .2809 \\ \end{array}$

DIET ANALYSIS FOR CONSUMER ODHE ON DATE 073076 WITH 100. OBSERVED FIELD, 5 SLIDES AND 6 FOOD SPECIES

$\begin{array}{lcc}\text { GM-3 } & & \text { RADIATION } \\ \text { DIET SAMPLE } & & \text { SAMPLE NO. } \\ 68 & & 421 \\ \text { FOOD SPECIES } & \text { FREQUENCY } & \text { DENSITY } \\ \text { SALX } & 69.0000 & 1.1712 \\ \text { MELI } & 35.0000 & .4308 \\ \text { SPHA } & 18.0000 & .1985 \\ \text { BROM } & 3.0000 & .0305 \\ \text { SOLI } & 3.0000 & .0305 \\ \text { EQUI } & 1.0000 & .0101 \\ & & \\ \text { FOOD SPECIES } & \text { REL FREQ } & \text { REL DENS } \\ \text { SALX } & 53.4884 & 62.5837 \\ \text { MELI } & 27.1318 & 23.0195 \\ \text { SPHA } & 13.9535 & 10.6045 \\ \text { BROM } & 2.3256 & 1.6276 \\ \text { SOLI } & 2.3256 & 1.6276 \\ \text { EQUI } & .7752 & .5371\end{array}$


DIET ANALYSIS FOR CONSUMER ODHE ON DATE 073076 WITH 100. OBSERVED FIELDS, 5 SLIDES AND 8 FOOD SPECIES

$\begin{array}{lcc}\begin{array}{c}\text { GM-3 } \\ \text { DIET SAMPLE }\end{array} & \begin{array}{c}\text { RADIATION J. } \\ \text { SAMPLE NO. }\end{array} \\ \text { FOOD SPECIES } & \text { FREQUENCY } & 427 \\ \text { SOLI } & 91.0000 & 2.4079 \\ \text { BAHY } & 14.0000 & .1508 \\ \text { LACT } & 2.0000 & .0202 \\ \text { RAPH } & 2.0000 & .0202 \\ \text { MELI } & 5.0000 & .0513 \\ \text { BROM } & 1.0000 & .0101 \\ \text { SALX } & 1.0000 & .0101 \\ \text { SPHA } & 1.0000 & .0101 \\ & & \\ \text { FOOD SPECIES } & \text { REL FREQ } & \text { REL DENS } \\ \text { SOLI } & 77.7778 & 89.8280 \\ \text { BAHY } & 11.9658 & 5.6264 \\ \text { LACT } & 1.7094 & .7537 \\ \text { RAPH } & 1.7094 & .7537 \\ \text { MELI } & 4.2735 & 1.9135 \\ \text { BROM } & .8547 & .3749 \\ \text { SALX } & .8547 & .3749 \\ \text { SPHA } & .8547 & .3749\end{array}$

DIET ANALYSIS FOR CONSUMER ODHE ON DATE 073076 WITH 100. OBSERVED FIELDS, 5 SLIDES AND 6 FOOD SPECIES

$\begin{array}{lcc}\begin{array}{c}\text { GM-4 } \\ \text { DIET SAMPLE }\end{array} & & \begin{array}{c}\text { RADIATION } \\ \text { SAMPLE NO. }\end{array} \\ \text { FOOD SPECIES } & & 439 \\ \text { FREQUENCY } & \text { DENSITY } \\ \text { SOLI } & 47.0000 & .6349 \\ \text { SALX } & 39.0000 & .4943 \\ \text { BAHY } & 7.0000 & .0726 \\ \text { PANI } & 1.0000 & .0101 \\ \text { BROM } & 4.0000 & .0408 \\ \text { MELI } & 11.0000 & .1165 \\ & & \\ \text { FOOD SPECIES } & \text { REL FREQ } & \text { REL DENS } \\ \text { SOLI } & 43.1193 & 46.3702 \\ \text { SALX } & 35.7798 & 36.1024 \\ \text { BAHY } & 6.4220 & 5.3004 \\ \text { PANI } & .9174 & .7341 \\ \text { BROM } & 3.6697 & 2.9816 \\ \text { MELI } & 10.0917 & 8.5114\end{array}$

DIET ANALYSIS FOR CONSUMER ODHE ON DATE 073076 WITH 100. OBSERVED FIELDS, 5 SLIDES AND 8 FOOD SEPCIES

$\begin{array}{lcc}\text { GM-3 } & & \text { RADIATION } \\ \text { DIET SAMPLE } & & \text { SAMPLE NO. } \\ 70 & & 422 \\ \text { FOOD SPECIES } & \text { FREQUENCY } & \text { DENSITY } \\ \text { SALX } & 82.0000 & 1.7148 \\ \text { MELI } & 7.0000 & .0726 \\ \text { SOLI } & 21.0000 & .2357 \\ \text { SIAL } & 1.0000 & .0101 \\ \text { OENO } & 2.0000 & .0202 \\ \text { BAHY } & 1.0000 & .0101 \\ \text { BROM } & 1.0000 & .0101 \\ \text { SAKA } & 1.0000 & .0101 \\ & & \\ \text { FOOD SPECIES } & \text { REL FREQ } & \text { REL DENS } \\ \text { SALX } & 70.6897 & 82.3039 \\ \text { MELI } & 6.0345 & 3.4831 \\ \text { SOLI } & 18.1034 & 11.3138 \\ \text { SIAL } & .8621 & .4824 \\ \text { OENO } & 1.7241 & .9697 \\ \text { BAHY } & .8621 & .4824 \\ \text { BROM } & .8621 & .4824 \\ \text { SAKA } & .8621 & .4824\end{array}$

DIET ANALYSIS FOR CONSUMER ODHE ON DATE 073076 WITH 100. OBSERVED FIELDS, 5 SLIDES AND 8 FOOD SPECIES

$\begin{array}{lcc}\begin{array}{c}\text { GM-4 } \\ \text { DIET SAMPLE }\end{array} & & \begin{array}{c}\text { RADIATION } \\ \text { SAMPLE NO. }\end{array} \\ \text { FOOD SPECIES } & \text { FREQUENCY } & 438 \\ \text { SENSITY } \\ \text { SPHA } & 58.0000 & .8675 \\ \text { SOLI } & 37.0000 & .4620 \\ \text { MELI } & 15.0000 & .1625 \\ \text { OENO } & 26.0000 & .3011 \\ \text { LACT } & 1.0000 & .0101 \\ \text { BROM } & 1.0000 & .0101 \\ \text { BAHY } & 1.0000 & .0101 \\ & 1.0000 & .0101 \\ \text { FOOD SPECIES } & & \\ \text { SALX } & R E L \mathrm{FREQ} & \text { REL DENS } \\ \text { SPHA } & 41.4286 & 47.3175 \\ \text { SOLI } & 26.4286 & 25.2015 \\ \text { MELI } & 10.7143 & 8.8645 \\ \text { OENO } & 18.5714 & 16.4237 \\ \text { LACT } & .7143 & .5482 \\ \text { BROM } & .7143 & .5482 \\ \text { BAHY } & .7143 & .5482 \\ \end{array}$


DIET ANALYSIS FOR CONSUMER ODHE ON DATE 073070́ WITH 100. OBSERVED FIELDS, 5 SLIDES AND 4 FOOD SPECIES

$\begin{array}{lcc}\text { GM-4 } & & \text { RADIATION } \\ \text { DIET SAMPLE } & & \text { SAMPLE NO. } \\ 73 & & 436 \\ \text { FOOD SPECIES } & \text { FREQUENCY } & \text { DENSITY } \\ \text { SALX } & 80.0000 & 1.6094 \\ \text { MELI } & 45.0000 & .5978 \\ \text { SOLI } & 3.0000 & .0305 \\ \text { CARE } & 1.0000 & .0701 \\ & & \\ \text { FOOD SPECIES } & \text { REL FREQ } & \text { REL DENS } \\ \text { SALX } & 62.0155 & 71.6017 \\ \text { MELI } & 34.8837 & 26.5967 \\ \text { SOLI } & 2.3256 & 1.3551 \\ \text { CARE } & .7752 & .4471\end{array}$

DIET ANALYSIS FOR CONSUMER ODHE ON DATE 073076 WITH 100. OBSERVED FIELDS, 5 SLIDES AND 7 FOOD SPECIES

$\begin{array}{lcc}\text { GM-4 } & & \text { RADIATION } \\ \text { DIET SAMPLE } & & \text { SAMPLE NO. } \\ 74 & & 435 \\ \text { FOOD SPECIES } & \text { FREQUENCY } & \text { DENSITY } \\ \text { SALX } & 60.0000 & .9163 \\ \text { BROM } & 31.0000 & .3717 \\ \text { SOLI } & 14.0000 & .1508 \\ \text { SAKA } & 20.0000 & .2231 \\ \text { MELI } & 5.0000 & .0513 \\ \text { BAHY } & 3.0000 & .0305 \\ \text { SEED } & 4.0000 & .0408 \\ & & \\ \text { FOOD SPECIES } & \text { REL FREQ } & \text { REL DENS } \\ \text { SALX } & 43.7956 & 51.3646 \\ \text { BROM } & 22.6277 & 20.8008 \\ \text { SOLI } & 10.2190 & 8.4547 \\ \text { SAKA } & 14.5985 & 12.5088 \\ \text { MELI } & 3.6496 & 2.8754 \\ \text { BAHY } & 2.1898 & 1.7075 \\ \text { SEED } & 2.9197 & 2.2884\end{array}$

DIET ANALYSIS FOR CONSUMER ODHE ON DATE 073076 WITH 100. OBSERVED FIELDS, 5 SLIDES AND 8 FOOD SPECIES

$\begin{array}{lcc}\text { GM-4 } & & \text { RADIATION } \\ \text { DIET SAMPLE } & & \text { SAMPLE NO. } \\ 75 & & 431 \\ \text { FOOD SPECIES } & \text { FREQUENCY } & \text { DENSITY } \\ \text { MELI } & 15.0000 & .1625 \\ \text { SOLI } & 77.0000 & 1.4697 \\ \text { SALX } & 1.0000 & .0101 \\ \text { SAKA } & 48.0000 & .6539 \\ \text { BROM } & 4.0000 & .0408 \\ \text { UNKG } & 1.0000 & .0101 \\ \text { BAHY } & 1.0000 & .0101 \\ \text { ROM } & 10.0000 & .7054 \\ & & \\ \text { FOOD SPECIES } & R E L F R E Q & \text { REL DENS } \\ \text { MELI } & 9.5541 & 6.5999 \\ \text { SOLI } & 49.0446 & 59.6834 \\ \text { SALX } & .6369 & .4081 \\ \text { SAKA } & 30.5732 & 26.5559 \\ \text { BROM } & 2.5478 & 1.6578 \\ \text { UNKG } & .6369 & .4081 \\ \text { BAHY } & .6369 & .4081 \\ \text { ROM } & 6.3694 & 4.2787\end{array}$

DIET ANALYSIS FOR CONSUMER ODHE ON DATE 073076 WITH 100. OBSERVED FIELDS, 5 SLIDES AND 8 FOOD SPECIES

$\begin{array}{lcc}\text { CM-4 } & & \text { RADIATION } \\ \text { DIET SAMPLE } & & \text { SAMPLE NO. } \\ 76 & & 437 \\ \text { FOOD SPECIES } & \text { FREQUENCY } & \text { DENSITY } \\ \text { MELI } & 40.0000 & .5108 \\ \text { SALX } & 72.0000 & 1.2730 \\ \text { OENO } & 1.0000 & .0101 \\ \text { BAHY } & 4.0000 & .0408 \\ \text { SOLI } & 8.0000 & .0834 \\ \text { BROM } & 1.0000 & .0101 \\ \text { CARE } & 2.0000 & .0202 \\ \text { MOSS } & 1.0000 & .0101 \\ & & \\ \text { FOOD SPECIES } & \text { REL FREQ } & \text { REL DENS } \\ \text { MELI } & 37.0078 & 26.0845 \\ \text { SALX } & 55.8140 & 65.0020 \\ \text { OENO } & .7752 & .5132 \\ \text { BAHY } & 3.1008 & 2.0845 \\ \text { SOLI } & 6.2016 & 4.2578 \\ \text { BROM } & .7752 & .5132 \\ \text { CARE } & 1.5504 & 1.0316 \\ \text { MOSS } & .7752 & .5132 \\ & & \end{array}$


DIET ANALYSIS FOR CONSUMER ODHE ON DATE 073076 WITH 100. OBSERVED FIELDS, 5 SLIDES AND 7 FOOD SPECIES

$\begin{array}{lcc}\begin{array}{c}\text { GM-4 } \\ \text { DIET SAMPLE } \\ 77\end{array} & \text { RADIATION } \\ \text { SAMPLE NO. } \\ \text { FOOD SPECIES } & \text { FREQUENCY } & 439 \\ \text { SALX } & 94.0000 & \text { DENSITY } \\ \text { SOLI } & 23.0000 & 2.8134 \\ \text { SEED } & 8.0000 & .2614 \\ \text { ASTR } & 9.0000 & .0834 \\ \text { BROM } & 1.0000 & .0943 \\ \text { MELI } & 1.0000 & .0101 \\ \text { UNKF } & 1.0000 & .0101 \\ & & .0101 \\ \text { FOOD SPECIES } & \text { REL FREQ } & \\ \text { SALX } & 68.6131 & \text { REL DENS } \\ \text { SOLI } & 16.7883 & 85.7063 \\ \text { SEED } & 5.8394 & 7.9621 \\ \text { ASTR } & 6.5693 & 2.5401 \\ \text { BROM } & .7299 & 2.8730 \\ \text { MELI } & .7299 & .3062 \\ \text { UNKF } & .7299 & .3062 \\ & & .3062\end{array}$

DIET ANALYSIS FOR CONSUMER ODHE ON DATE 073076 WITH 100. OBSERVED FIELDS, 5 SLIDES AND 5 FOOD SPECIES

$\begin{array}{lcc}\text { CM-4 } & & \text { RADIATION } \\ \text { DIET SAMPLE } & & \text { SAMPLENO. } \\ 79 & & 434 \\ \text { FOOD SPECIES } & \text { FREQUENCY } & \text { DENSITY } \\ \text { SALX } & 100.0000 & \ldots * * * * \\ \text { MELI } & 5.0000 & .0513 \\ \text { BROM } & 3.0000 & .0305 \\ \text { SPHA } & 1.0000 & .0101 \\ \text { EQUI } & 1.0000 & .0101 \\ & & \\ \text { FOOD SPECIES } & \text { REL FREQ } & \text { REL DENS } \\ \text { SALX } & 90.9091 & \mathbf{* * * * *} \\ \text { MELI } & 4.5455 & 50.3600 \\ \text { BROM } & 2.7273 & 29.9095 \\ \text { SPHA } & .9091 & 9.8675 \\ \text { EQUI } & .9091 & 9.8675\end{array}$

DIET ANALYSIS FOR CONSUMER ODHE ON DATE 073076 WITH 100. OBSERVED FIELDS, 5 SLIDES AND 6 FOOD SPECIES

$\begin{array}{lcc}\text { GM-4 } & & \text { RADIATION } \\ \text { DIET SAMPLE } & & \text { SAMPLE NO. } \\ 78 & & 440 \\ \text { FOOD SPECIES } & \text { FREQUENCY } & \text { DENSITY } \\ \text { SALX } & 71.0000 & 1.2379 \\ \text { SOLI } & 18.0000 & .1985 \\ \text { BROM } & 4.0000 & .0408 \\ \text { MELI } & 9.0000 & .0943 \\ \text { PHLO } & 2.0000 & .0202 \\ \text { GRSP } & 1.0000 & .0101 \\ & & \\ \text { FOOD SPECIES } & \text { REL FREQ } & \text { REL DENS } \\ \text { SALX } & 67.6190 & 77.2845 \\ \text { SOLI } & 17.1429 & 12.3899 \\ \text { BROM } & 3.8095 & 2.5486 \\ \text { MELI } & 8.5714 & 5.8881 \\ \text { PHLO } & 1.9048 & 1.2613 \\ \text { GRSP } & .9524 & .6275\end{array}$

DIET ANALYSIS FOR CONSUMER ODHE ON DATE 073076 WITH 100. OBSERVED FIELDS. 5 SLIDES AND 7 FOOD SPECIES

$\begin{array}{lcc}\text { GM-4 } & & \text { RADIATION } \\ \text { DIET SAMPLE } & & \text { SAMPLE NO. } \\ 80 & & 432 \\ \text { FOOD SPECIES } & \text { FREQUENCY } & \text { DENSITY } \\ \text { SOLI } & 53.0000 & .7550 \\ \text { EQUI } & 10.0000 & .1054 \\ \text { SALX } & 15.0000 & .1625 \\ \text { SAKA } & 22.0000 & .2485 \\ \text { BAHY } & 4.0000 & .0408 \\ \text { MELI } & 3.0000 & .0305 \\ \text { BROM } & 1.0000 & .0101 \\ & & \\ \text { FOOD SPECIES } & \text { REL FREQ } & \text { REL DENS } \\ \text { SOLI } & 49.0741 & 55.8162 \\ \text { EQUI } & 9.2593 & 7.7889 \\ \text { SALX } & 13.8889 & 12.0145 \\ \text { SAKA } & 20.3704 & 18.3679 \\ \text { BAHY } & 3.7037 & 3.0178 \\ \text { MELI } & 2.7778 & 2.2517 \\ \text { BROM } & .9259 & .7430\end{array}$


DIET ANALYSIS FOR CONSUMER ODHE ON DATE 080476 WITH 100. OBSERVED FIELDS, 5 SLIDES AND 5 FOOD SPECIES

$B C-1$

DIET SAMPLE

81

FOOD SPECIES

PUTR

SALX

OENO

SPHA

POA

FOOD SPECIES

PUTR

SALX

OENO

SPHA

POA
RADIATION

SAMPLE NO.

448

FREQUENCY

77.0000

34.0000

21.0000

2.0000

1.0000

REL FREQ

57.0370

25.7852

15.5556

1.4815

.7407
DENSITY

1.4697

.4155

.2357

.0202

.0101

REL DENS

68.3199

19.3158

10.9579

.9392

.4672
DIET ANALYSIS FOR CONSUMER ODHE ON DATE 080476 WITH 100. OBSERVED FIELDS, 5 SLIDES AND 5 FOOD SPECIES

BC- 1
DIET SAMPLE
82
FOOD SPECIES
PUTR
OENO
SALX
SOLI
SAKA

FREQUENCY

46.0000

59.0000

15.0000

2.0000

4.0000

RADIATION

SAMPLE NO.

444

DEN5ITY

.6162

.8916

.1625

.0202

.0408

FOOD SPECIES

PUTR

OENO

SALX

SOL!

SAKA

REL FREQ

REL DENS

36.5079

46.8254

11.9048

1.5873

3.1746
35.5904

51.4979

9.3870

1.1669

2.3578
DIET ANALYSIS FOR CONSUMER ODHE ON DATE 080476 WITH 100. OBSERVED FIELDS, 5 SLIDES AND 3 FOOD SPECIES

$B C-1$

DIET SAMPLE

83

FOOD SPECIES

PUTR

SAKA

ASTR

FOOD SPECIES

PUTR

SAKA

ASTR
RADIATION

SAMPLE NO.

445

DENSITY

100.0000

5.0000

2.0000

REL FREQ

93.4579

4.6729

1.8692
.0513

.0202

REL DENS

$* * * * * * *$

71.7429

28.2571
DIET ANALYSIS FOR CONSUMER ODHE ON DATE 080476 WITH 100. OBSERVED FIELDS, 5 SLIDES AND 3 FOOD SPECIES

$B C-1$

DIET SAMPLE

84

FOOD SPECIES

PUTR

SALX

SOLI

FOOD SPECIES

PUTR

SALX

SOLI
RADIATIONO. SAMPLE NO.

443

DENSITY

100.0000

2.0000

1.0000

.0202

.0101

REL FREQ

97.0874

1.9417

.9709
REL DENS

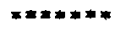

66.7791

33.2209 
DIET ANALYSIS FOR CONSUMER ODHE ON DATE 680476 WITH 100. OBSERVED FIELDS, 5 SLIDES AND 5 FOOD SPECIES

8C-1

DIET SAMPLE

85

FOOD SPECIES

PUTR

BROM

SAKA

SALX

OENO

FOOD SPECIES

PUTR

BROM

SAKA

SALX

OENO
RADIATION

SAMPLE NO. 450

FREQUENCY 100.000 1.0000 3.0000 4.0000

2.0000

REL FREQ

90.9091

.9091

2.7273

3.6364

1.8182
DENSITY

0101

.0305

.0408

.0202

REL DENS

*******

29.9990

40.2051

19.8974
DIET ANALYSIS FOR CONSUMER ODHE ON DATE 080476 WITH 100. OBSERVED FIELDS, 5 SLIDES AND 5 FOOD SPECIES

$B C-1$

DIET SAMPLE

86

FOOD SPECIES

SALX

SOLI

MELI

SAKA

BROM

FOOD SPECIES

SALX

SOLI

MELI

SAKA

BROM
RADIATION

SAMPLE NO.

446

DENSITY

3.2189

.0408

.0305

.0305

.0101

REL DENS

96.6436

1.2256

.9145

.9145

.3018
DIET ANALYSIS FOR CONSUMER ODHE ON DATE 080476 WITH 100. OBSERVED FIELDS, 5 SLIDES 3 FOOD SPECIES

$B C-1$

DIET SAMPLE

87

FOOD SPECIES

PUTR

BROM

SALX

FOOD SPECIES

PUTR

BROM

SALX
RADIATION

SAMPLE NO.

441

FREQUENCY

100.0000

1.0000

1.0000

REL. FREQ

98.0392

.9804

.9804

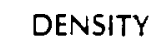

.0101

.0101

REL DENS

$* * * * * *$

50.0000

50.0000
DIET ANALYSIS FOR CONSLMER ODHE ON DATE 080476 WITH 100. OBSERVED FIELDS, 5 SLIDES AND 4 FOOD SPECIES

\begin{tabular}{lc}
\multicolumn{2}{c}{ BC-1 } \\
DIET SAMPLE \\
$\quad 88$ \\
FOOD SPECIES & FREQUENCY \\
PUTR & 94.0000 \\
SOLI & 3.0000 \\
OENO & 3.0000 \\
SALX & 3.0000 \\
& \\
FOOD SPECIES & REL FREQ \\
PUTR & 91.2621 \\
SOLI & 2.9126 \\
OENO & 2.9126 \\
SALX & 2.9126
\end{tabular}

RADIATION SAMPLE NO. 447

DENSITY

2.8134

.0305

.0305

.0305

REL DENS

96.8542

1.0486

1.0486

1.0486 
DIET ANALYSIS FOR CONSUMER ODHE ON DATE 080476 WITH 100. OBSERVED FIELDS, 5 SLIDES AND 4 FOOD SPECIES

$\begin{array}{lcc}\text { BC-1 } & & \text { RADIATION } \\ \text { DIET SAMPLE } & & \text { SAMPLE NO. } \\ 89 & & 449 \\ \text { FOOD SPECIES } & \text { FREQUENCY } & \text { DENSITY } \\ \text { PUTR } & 66.0000 & 1.0788 \\ \text { OENO } & 34.0000 & .4155 \\ \text { SALX } & 8.0000 & .0834 \\ \text { SOLI } & 2.0000 & .0202 \\ & & \\ \text { FOOD SPECIES } & \text { REL FREQ } & \text { REL DENS } \\ \text { PUTR } & 60.0000 & 67.5138 \\ \text { OENO } & 30.9091 & 26.0037 \\ \text { SALX } & 7.2727 & 5.2182 \\ \text { SOLI } & 1.8182 & 1.2643\end{array}$

DIET ANALYSIS FOR CONSUMER ODHE ON DATE 080476 WITH 100. OBSERVED FIELDS, 5 SLIDES AND 8 FOOD SPECIES

$B C-2$

DIET SAMPLE

91

FOOD SPECIES

$S A L X$

MELI

PL'TR

VETH

SOLI

BROM

OENO

SAKA

FOOD SPECIES

SALX

MELI

PUTR

VETH

SOLI

BROM

OENO

SAKA
RADIATION SAMPLE NO.

455

FREQUENCY

30.0000

37.0000

40.0000

1.0000

4.0000

2.0000

2.0000

1.0000

REL FREQ

25.6410

37.6239

34.1880

.8547

3.4188

1.7094

1.7094

.8547
BC-1

DIET SAMPLE

90

FOOD SPECIES

PUTR

SALX

SAKA

OENO

FOOD SPECIES

PUTR

SALX

SAKA

OENO
RADIATION

SAMPLE NO.

442

FREQUENCY

100.0000

8.0000

4.0000

1.0000

REL FREQ

88.4956

7.0796

3.5398

.8850
DENSITY

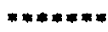

.0834

.0408

.0101

REL DENS

*******

62.1074

30.4066

7.4861
DIET ANALYSIS FOR CONSUMER ODHE ON DATE 080476 WITH 100. OBSERVED FIELDS, 5 SLIDES AND 6 FOOD SPECIES

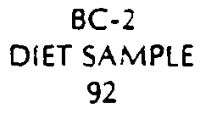

$B C-2$

DIET SAMPLE

92

RADIATION
SAMPLE NO.

$$
453
$$

FOOD SPECIES

$S A L X$

PUTR

SEED

SAKA

OENO

SOLI

FOOD SPECIES

SALX

PUTR

SEED

SAKA

OENO

SOLI
FREQUENCY

38.0000

61.0000

23.0000

8.0000

5.0000

1.0000

REL FREQ

27.9412

44.8529

16.9118

5.8824

3.6765

.7353
DENSITY

.4780

.9416

.2614

.0834

.0513

.0101

REL DENS

26.1832

51.5742

14.3156

4.5670

2.8095

.5505 
DIET ANALYSIS FOR CONSUMER ODHE ON DATE 080476 WITH 100. OBSERVED FIELDS, 5 SLIDES AND 3 FOOD SPECIES

$B C-2$

DIET SAMPLE

93

FOOD SPECIES

PUTR

OENO

SOLI

FOOD SPECIES

PUTR

OENO

SOLI
RADIATION

SAMPLE NO.

452

DENSITY

.1863

.0202

REL DENS

REL FREQ

84.0336

14.2857

1.6807
90.2181

9.7879
DIET ANALYSIS FOR CONSUMER ODHE ON DATE 080476 WITH 100. OBSERVED FIELDS, 5 SLIDES AND 4 FOOD SPECIES

BC-2
DIET SAMPLE
94
FOOD SPECIES
PUTR
OENO
SOLI
SAKA
FOOD SPECIES
PUTR
OENO
SOLI
SAKA

FREQUENCY
100.0000
25.0000
1.0000
6.0000
REL FREQ
75.7576
18.9394
.7576
4.5455

RADIATION

SAMPLE NO.

451

DENSITY

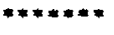

.2877

.0101

.0619

REL DENS

79.9988

2.7948

17.2064
DIET ANALYSIS FOR CONSUMER ODHE ON DATE 080476 WITH 100. OBSERVED FIELDS, 5 SLIDES AND 5 FOOD SPECIES

$\begin{array}{lcc}\text { BC-2 } & & \text { RADIATION } \\ \text { DIET SAMPLE } & & \begin{array}{c}\text { SAMPLE NO. } \\ 456\end{array} \\ 95 & & \text { DENSITY } \\ \text { FOOD SPECIES } & \text { FREQUENCY } & * * * * * * \\ \text { PUTR } & 100.0000 & .1054 \\ \text { SAKA } & 10.0000 & .0305 \\ \text { SOLI } & 3.0000 & .0101 \\ \text { SALX } & 1.0000 & .0101 \\ \text { OENO } & 1.0000 & \\ & & \text { REL DENS } \\ \text { FOOD SPECIES } & \text { REL FREQ } & * * * * * * \\ \text { PUTR } & 86.9565 & 67.5733 \\ \text { SAKA } & 8.6957 & 19.5351 \\ \text { SOLI } & 2.6087 & 6.4458 \\ \text { SALX } & .8696 & 6.4458 \\ \text { OENO } & .8696 & \end{array}$

DIET ANALYSIS FOR CONSUMER ODHE ON DATE 080476 WITH 100. OBSERVED FIELDS, 5 SLIDES AND 2 FOOD SPECIES

\begin{tabular}{|c|c|}
\hline $\begin{array}{c}\text { BC-2 } \\
\text { DIET SAMPLE } \\
96\end{array}$ & \\
\hline $\begin{array}{l}\text { FOOD SPECIES } \\
\text { PUTR } \\
\text { SALX }\end{array}$ & $\begin{array}{l}\text { FREQUENCY } \\
99.0000 \\
8.0000\end{array}$ \\
\hline $\begin{array}{l}\text { FOOD SPECIES } \\
\text { PLTR } \\
\text { SALX }\end{array}$ & $\begin{array}{c}\text { REL FREQ } \\
92.5234 \\
7.4766\end{array}$ \\
\hline
\end{tabular}

RADIATION
SAMPLE NO.
460
DENSITY
4.6052
.0834

REL DENS
98.2216
1.7784


DIET ANALYSIS FOR CONSUMER ODHE ON DATE 080476 WITH 100. OBSERVED FIELDS. 5 SLIDES AND 5 FOOD SPECIES

$\begin{array}{lcc}\text { BC-2 } & & \text { RADIATION } \\ \text { DIET SAMPLE } & & \begin{array}{c}\text { SAPLE NO. } \\ 97\end{array} \\ \text { FOOD SPECIES } & \text { FREQUENCY } & \begin{array}{c}454 \\ \text { DENSITY }\end{array} \\ \text { PUTR } & 100.0000 & * * * * * * * \\ \text { SALX } & 31.0000 & .3711 \\ \text { OENO } & 2.0000 & .0202 \\ \text { SEED } & 1.0000 & .0101 \\ \text { SAKA } & 5.0000 & .0513 \\ & & \\ \text { FOOD SPECIES } & \text { RELFREQ } & \text { REL DENS } \\ \text { PUTR } & 71.9424 & * * * * * * \\ \text { SALX } & 22.3022 & 81.9831 \\ \text { OENO } & 1.4388 & 4.4636 \\ \text { SEED } & .7194 & 2.2205 \\ \text { SAKA } & 3.5971 & 11.3328\end{array}$

DIET ANALYSIS FOR CONSUEMR ODHE ON DATE 080476 WITH 100. OBSERVED FIELDS, 5 SLIDES AND 7 FOOD SPECIES

$\begin{array}{lcc}\text { BC-2 } & & \text { RADIATION } \\ \text { DIET SAMPLE } & & \begin{array}{c}\text { SAMPLE NO. } \\ 99\end{array} \\ \text { FOOD SPECIES } & \text { FREQUENCY } & \text { DENSITY } \\ \text { SALX } & 71.0000 & 1.2379 \\ \text { MELI } & 25.0000 & .2877 \\ \text { PUTR } & 4.0000 & .0408 \\ \text { SAKA } & 4.0000 & .0408 \\ \text { SOLI } & 5.0000 & .0513 \\ \text { STIP } & 1.0000 & .0101 \\ \text { OENO } & 1.0000 & .0101 \\ & & \\ \text { FOOD SPECIES } & \text { REL FREQ } & \text { REL DENS } \\ \text { SALX } & 63.9640 & 73.7447 \\ \text { MELI } & 22.5225 & 17.1383 \\ \text { PLTR } & 3.6036 & 2.4319 \\ \text { SAKA } & 3.6036 & 2.4319 \\ \text { SOLI } & 4.5045 & 3.055 \% \\ \text { STIP } & .9009 & .5987 \\ \text { OENO } & .9009 & .5987\end{array}$

DIET ANALYSIS FOR CONSUMER ODHE ON DATE 080476 WITH OBSERVED FIELDS, 5 SLIDES AND 4 FOOD SPECIES

$\begin{array}{ccc}\text { BC-2 } & & \text { RADIATION } \\ \text { DIET SAMPLE } & & \text { SAMPLE NO. } \\ 98 & & 459 \\ \text { FOOD SPECIES } & \text { FREQUENCY } & \text { DENSITY } \\ \text { PUTR } & 100.0000 & * * * * * * \\ \text { SAKA } & 2.0000 & .0202 \\ \text { SALX } & 2.0000 & .0202 \\ \text { MELI } & 4.0000 & .0408 \\ & & \\ \text { FOOD SPECIES } & \text { REL FREQ } & \text { REL DENS } \\ \text { PUTR } & 92.5926 & * * * * * * \\ \text { SAKA } & 1.8519 & 24.8718 \\ \text { SALX } & 1.8519 & 24.8718 \\ \text { MELI } & 3.7037 & 50.2564\end{array}$

DIET ANALYSIS FOR CONSUMER ODHE ON DATE 080476 WITH 100. OBSERVED FIELDS, 5 SLIDES AND 3 FOOD SPECIES

$\begin{array}{lcc}\text { BC-2 } & & \text { RADIATION } \\ \text { DIET SAMPLE } & & \text { SAMPLE NO. } \\ 100 & & 458 \\ \text { FOOD SPECIES } & \text { FREQUENCY } & \text { DENSITY } \\ \text { PUTR } & 100.000 & * * * * * * \\ \text { SALX } & 9.0000 & .0943 \\ \text { SAKA } & 2.0000 & .0202 \\ & & \\ \text { FOOD SPECIES } & \text { REL FREQ } & \text { REL DENS } \\ \text { PUTR } & 90.0901 & \ldots * * * * * \\ \text { SALX } & 8.1081 & 82.3578 \\ \text { SAKA } & 1.8018 & 17.6422\end{array}$




\section{Computer Diet Analysis Program}

1

10

15

20

C... DENS IS INITIALIZED TO A VALUE THAT WILL BLOW THE OUTPUT FORMAT TO

C... INDICATE WHEN FREQUENCY WAS 100 PERCENT AND DENSITY WAS INDETERMINATE.

C...

C...

C...

C...

C...

C...

C...

85

10

30

25

32

100

30

35

105

40

15

C..

PROGR,AM DIET (INPLT, OUTPUT, TAPES=INPUT, TAPEG=OUTPUT)

DIMENSION FREQ (100), DENS (100, NAME (100), ISPS (28), IDATA (28)

DIMENSION DATA $(28)$

UP TO 4 CARDS MAY BE USED FOR EACH SLIDE. LAST CARD FOR SLIDE

MUST HAVE A BLANK IN COL 80. OTHER CARDS FOR SAME SLADE MUST HAVE A 1 IN COL 80.

PLACE A BLANK CARD AT THE END OF EACH GROUP (CONSUMER SPECIES,

DATE OR WHATEVER) TO BE ANALYZED.

INITIALIZE COUNTERS AND VARIABLES

TOTFQ=TOT DENS $=0$.

NSLIDS $=$ NNAME $=0$

TFLDS $=0$

DO $10 \mathrm{~J}=1,100$

FREQ $(J)=0$.

INDICATE WHEN
NAME (J) $=10 \mathrm{H}$

CONTINUE

CONTINUE

DO $32 \mathrm{Jl}=1,28$

ISPS $(J)=10 \mathrm{H}$

IDATA $(I J)=0$

CONTINUE

$\operatorname{READ}(5,100)$ MDATE, MCONS, IFLDS, (ISPS (J), IDATA (J), J=1,7), IFOLLOW

FORMAT (3X, A6, 6X, A4, 13,8X,7(A4,13), 11)

IF (EOF (5). NE. 0) CO TO 90

IF (IFOLLOW.NE. 9 ) READ $(5,105)$ (ISPS(J), IDATA (J), J=3,14), IFOLLOW

IF EOF (5) .NE. 0) STOP "ERROR 1"

IF (IFOLLOW. GT. 0)READ $(5,105)$ (ISPS (I), IDATA (J), J=15,21), IFOLLOW

IF EOF (5).NE. 9 ) STOP "ERROR 2"

IF (IFOLLOW. GT. 0) RE.AD $(5,105)$ (ISPS (J), IDATA (J),J=22,28), IFFOLLOW

IF ( EOF (5) .NE. O) STOP "ERROR 3"

FORMAT $(30 \times, 7$ (A4,13), 11)

IF (MCONS.EQ. $4 \mathrm{H}$ ) GO TO 35

NCONS $=$ MCONS

NDATE $=$ MDATE

DO $15 K=1.28$

DATA $(K)=$ IDATA $(K)$

TFLDS=TFLDS+IFLDS

COUNT SLIDES

NSLIDS=NSLIDS+1

C... NNAME IS THE NUMBER OF OBSERVED FOOD SPECIES, WHICH ARE STORED IN NAME

C... LOC IN THE FOOD SPECIES NAMES IF NECESSARY, AND ACCUMULATE OBSERVATIONS.

DO $20 \mathrm{~J}=1,28$

IF (ISPS (J).EQ.4H I GO TO 25

DO $40 \mathrm{JK}=1$, NNAME

$K E E P=J K$

IF (ISPS (J).EQ.NAME (JK)) GO TO 50

CONTINUE

NNAME=NNAME+1

NAME (NNAME) =ISPS (J)

FREQ (NNAME) $=$ DATA (J)

CO TO 20

50

FREQ (KEEP) $=F R E Q(K E E P)+D A T A(J)$ 


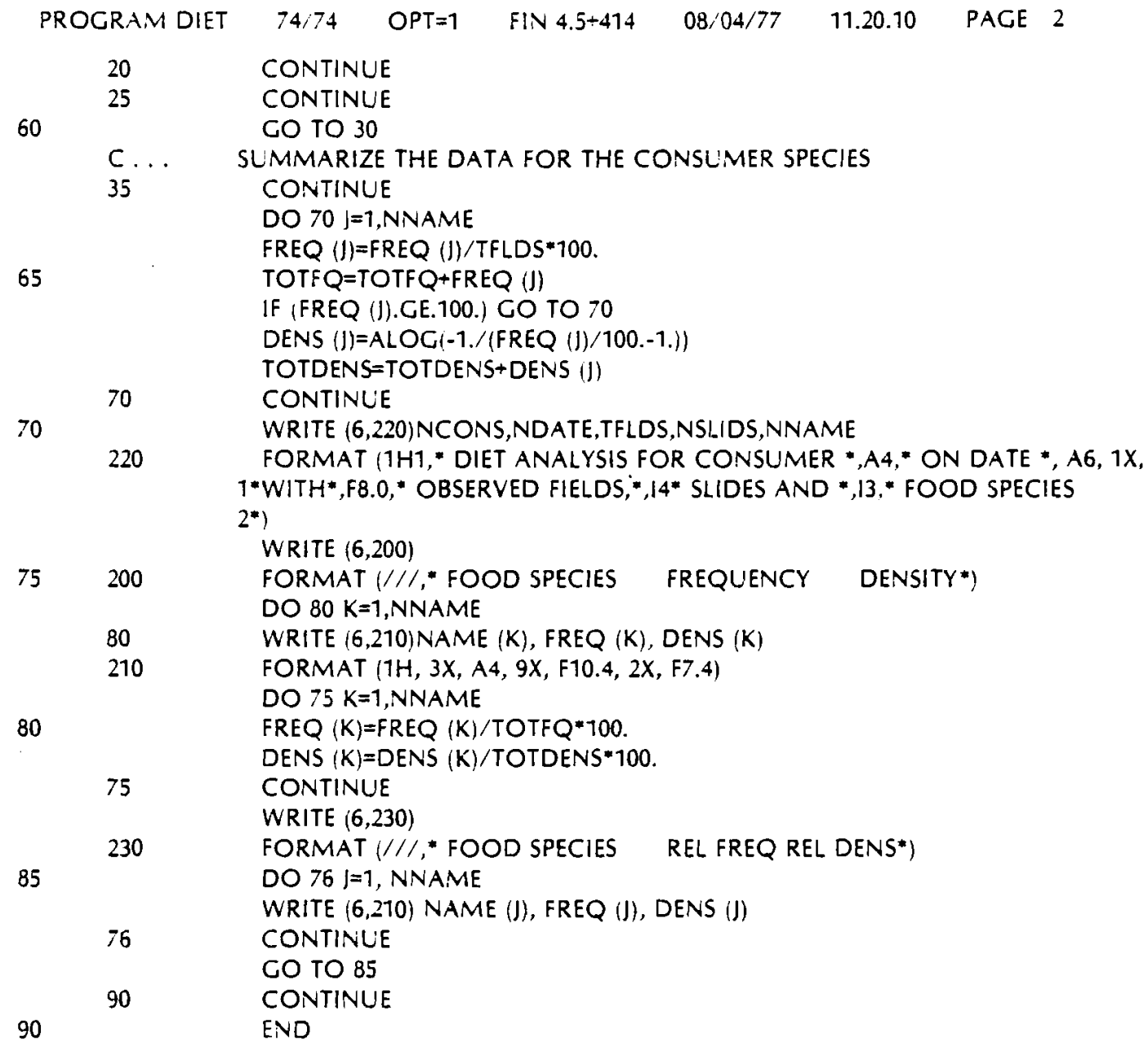

END 


\section{Original Diet Data}

B-Pond Site and Transect-Pellet Group No.

\begin{tabular}{|c|c|c|c|c|c|c|c|c|}
\hline 073076 & ODHE & 20BP1-0091SAKA & $20 E C C R$ & 2SOLI & 1 & & & \\
\hline 073076 & ODHE & 20BP1-00925AKA & 19ECCR & 1 & SEED & 1 & & \\
\hline 073076 & ODHE & 20BP1-00935AKA & 19 & SOLI & 1 & & & \\
\hline 073076 & ODHE & 20BP1-00945AKA & 20ECCR & 1 & SEED & 1 & & \\
\hline 073076 & ODHE & 20BP1-0095SAKA & 19 & & SEED & 1 & & \\
\hline 073076 & ODHE & 20BP1-0051SAKA & 20ECCR & 1SPHA & 2 & & & \\
\hline 073076 & ODHE & 20BP1-0055SAKA & $18 S O L I$ & 2SPHA & 3 & & & \\
\hline 073076 & ODHE & 20BP1-0054SAKA & 19 & SPHA & 2 & & & \\
\hline 073076 & ODHE & 20BP1-0053SAK,A & 19 & SPHA & 1 & & & \\
\hline 073076 & ODHE & 20BP1-0052SAKA & 20ECCR & 2SPHA & 3 & & & \\
\hline 073076 & ODHE & 20BP1-0021SAKA & 16SOLI & 8SEED & 1OENO & 1SHEP & 1 & \\
\hline 073076 & ODHE & 20BP1-0022SAKA & 16SOLI & 5 & BROM & 1SPHA & $1 E C C R$ & 3 \\
\hline 073076 & ODHE & 20BP1-0023SAKA & 17SOLI & 9SEED & 2BROM & 1SPHA & 1ECCR & 2 \\
\hline 073076 & ODHE & 20BP1-0024SAKA & $135 \mathrm{OLI}$ & 7SEED & 2 & SPHA & $3 E C C R$ & 3 \\
\hline 073076 & ODHE & 20BP1-0025SAKA & $185 \mathrm{OLI}$ & 6 & OENO & 1SPHA & 1 & \\
\hline 073076 & ODHE & 20BP1-0031SAKA & 19ECCR & 2BROM & 1SEED & 2 & & \\
\hline 073076 & ODHE & 20BP1-0032SAKA & 19SEED & 1 & & & & \\
\hline 073076 & ODHE & 20BP1-0033SAKA & 20POA & 1 & & & & \\
\hline 073076 & ODHE & 20BP1-0034SAKA & 19ECCR & 1SEED & 1 & & & \\
\hline 073076 & ODHE & 20BP1-0035SAKA & 20 & & & & & \\
\hline 073076 & ODHE & 20PB1-0011SALX & $25 A K A$ & 16ECCR & 2CHNA & 1 & & \\
\hline 073076 & ODHE & 208P1-0012SALX & 2SAKA & $6 \mathrm{ECCR}$ & 4 & & & \\
\hline 073076 & ODHE & 20BP1-0013SALX & 4SAKA & $17 E C C R$ & 1 & , & & \\
\hline 073076 & ODHE & 20BP1-0014SALX & 2SAKA & 16 & & 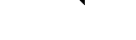 & & \\
\hline 073076 & ODHE & 20BP1-0015SALX & $3 S A K A$ & 18CHNiA & 2 & & & \\
\hline 073076 & ODHE & 20BP1-0071VETH & 14SAKA & $15 \mathrm{SOLI}$ & 11ECCR & 1SEED & 1 & \\
\hline 073076 & ODHE & 20BP1-0072VETH & 17 SAKA & 16SOLI & 12ECCR & 2 & & \\
\hline 073076 & ODHE & 20BP1-0073VETH & 13SAKA & $1650 \mathrm{LI}$ & TECCR & 3 & & \\
\hline 073076 & ODHE & 20BP1-0074VETH & 19VETH & $185 \mathrm{OL}$ & 9ECCR & $i$ & & \\
\hline 073076 & ODHE & 20BP1-0075VETH & $125 \mathrm{AKA}$ & $105 \mathrm{OLI}$ & 11ECCR & 2SEED & 1 & \\
\hline 073076 & ODHE & 20BP1-0081SAKA & 18SEED & & 4ECCR & 4SOLI & 2 & \\
\hline 073076 & ODHE & 20BP1-0082SAKA & 13SEED & 2BROM & 3ECCR & 5SOLI & 2 & \\
\hline 073076 & ODHE & 20BP1-0083SAKA & 14SEED & 1BROM & TECCR & 3SOLI & $3 S A L X$ & 2 \\
\hline 073076 & ODHE & 20BP1-0084SAKA & 16SEED & 1BROM & $4 E C C R$ & 8SALX & 1 & \\
\hline 073076 & ODHE & 20BP1-0085SAKA & 14SEED & 2BROM & 1ECCR & 5SOLI & 2 & \\
\hline 073076 & ODHE & 20BP1-0061SOLI & 11SAKA & 16SALX & $2 E C C R$ & 1BROM & 1SEED & 1 \\
\hline 073076 & ODHE & 20BP1-0062SOLI & 7SAKA & 13SALX & AECCR & ABROM & 1 & \\
\hline 073076 & ODHE & 20BP1-0063SOLI & 3SAKA & 17SALX & $1 E C C R$ & 4BROM & 1 & \\
\hline 073076 & ODHE & 20BP1-0064SOLI & $115 A K A$ & 12SALX & ECCR & 3BROM & 1SEED & 1 \\
\hline 073076 & ODHE & 20BP1-0065SOLI & 65AKA & 10SALX & $2 E C C R$ & 3 & & \\
\hline 073076 & ODHE & 20BP1-0041SAKA & 16SOLI & $3 E C C R$ & 1BROM & 1 & & \\
\hline 073076 & ODHE & 20BP1-0042SAKA & 17SAKA & 6ECCR & 1BROM & TSALX & 1 & \\
\hline 073076 & ODHE & 20BP1-0043SAKA & 19SOLI & JECCR & 2SEED & 1 & & \\
\hline 073076 & ODHE & 20BP1-0044SAKA & $1750 \mathrm{OL}$ & & 1 & & & \\
\hline 073076 & ODHE & 20BP1-0045SAKA & $195 \mathrm{OLI}$ & SECCR & 1SALX & 3 & & \\
\hline 073076 & ODHE & 20BP1-0101SALX & $12 \mathrm{ECCR}$ & 5SAKA & $6 \mathrm{SOLI}$ & 1 & & \\
\hline 073076 & ODHE & 20BP1-0102SALX & 10ECCR & TS.AKA & TSOLI & 2VETH & 1BROM & 1 \\
\hline 073076 & ODHE & 20BP1-0103SALX & 10ECCR & 3SAKA & 11SOLI & 3 & & \\
\hline 073076 & ODHE & 20BP1-01045ALX & SECCR & TSAKA & $12 \mathrm{SOLI}$ & 2 & & \\
\hline 073076 & ODHE & 20BP1-0105SALX & SECCR & BSAKA & 9SOLI & 1 & & \\
\hline
\end{tabular}




\begin{tabular}{|c|c|c|c|c|c|c|c|c|}
\hline 073076 & ODHE & 20BP2-0161SAKA & 18SALX & 3 & & & & \\
\hline 073076 & ODHE & 20BP2-0162SAKA & T9SALX & $2 \mathrm{ECCR}$ & 1 & & & \\
\hline 073076 & ODHE & 20BP2-0163SAKA & 18SALX & SECCR & 1SEED & 1 & & \\
\hline 073076 & ODHE & 20BP2-0164SAKA & 14SALX & 7ECCR & 2SEED & $2 \mathrm{POA}$ & 1 & \\
\hline 073076 & ODHE & 20BP2-0165SAKA & 19SALX & SECCR & 1 & & & \\
\hline 073076 & ODHE & 20BP2-0171SAKA & $18 S O L I$ & 1ECCR & 1DEPI & 1 & & \\
\hline 073076 & ODHE & 20BP2-0172SAKA & 19SOLI & 0 & DEPI & 1 & & \\
\hline 073076 & ODHE & 20BP2-0173SAKA & $185 \mathrm{OLI}$ & 1 & & & & \\
\hline 073076 & ODHE & 20BP2-0174SAKA & $1850 \mathrm{LI}$ & 2 & DEPI & 1 & & \\
\hline 073076 & ODHE & 20BP2-0175SAKA & 19SOLI & IECCR & 2 & & & \\
\hline 073076 & ODHE & 20BP2-0141SAKA & 20SEED & 2 & & & & \\
\hline 073076 & ODHE & 20BP2-0142SAKA & 19SEED & TECCR & 3 & & & \\
\hline 073076 & ODHE & 20BP2-0143SAKA & 18SEED & 2ECCR & 5SOLI & TMICR & 1 & \\
\hline 073076 & ODHE & 20BP2-0144SAKA & 18SEED & $3 E C C R$ & $15 O L I$ & $1 M I C R$ & 1 & \\
\hline 073076 & ODHE & 20BP2-0145SAKA & 18SEED & AECCR & 4 & & & \\
\hline 073076 & ODHE & 208P2-0111SPHA & 19SOLI & 3SEED & $2 S A K A$ & $3 E C C R$ & 1 & \\
\hline 073076 & ODHE & 20BP2-0112SPHA & $205 O L I$ & 3SEED & 1SAKA & $3 E C C R$ & 1 & \\
\hline 073076 & ODHE & 20BP2-0113SPHA & 19SOLI & 5 & SAKA & 2 & & \\
\hline 073076 & $\mathrm{ODHE}$ & 20BP2-0174SPHA & 18SOLI & 1SEED & 1SAKA & 2 & OENO & 1 \\
\hline 073076 & ODHE & 20BP2-0115SPHA & 19SOLI & 2SEED & 2SAKA & TECCR & IPOA & 1 \\
\hline 073076 & ODHE & 20BP2-0201SAKA & 16ASTR & 1ECCR & 7SEED & 2 & & \\
\hline 073076 & ODHE & $20 B P 2-02025 A K A$ & $16 P O A$ & 2ECCR & 5 & & & \\
\hline 073076 & ODHE & 20BP2-02035AKA & 16POA & $1 E C C R$ & 5 & & & \\
\hline 073076 & ODHE & 20BP2-0204SAKA & 15 & $E C C R$ & 35EED & 2 & & \\
\hline 073076 & ODHE & 20BP2-0205SAKA & 17EQUI & TECCR & 1SEED & 1SOLI & 1 & \\
\hline 073076 & ODHE & 20BP2-0191SPHA & 20SAKA & 1 & & & & \\
\hline 073076 & ODHE & 20BP2-0192SPHA & 19SAKA & 3BROM & 1 & & & \\
\hline 073076 & ODHE & 20BP2-0193SPHA & 20SAKA & 2 & & & & \\
\hline 073076 & ODHE & 20BP2-0194SPHA & 20SAKA & 1 & & & & \\
\hline 073076 & ODHE & 20BP2-0195SPHA & 20SAKA & TOENO & 1 & & & \\
\hline 073076 & ODHE & 20BP2-0181SAKA & 19OENO & $6 P O A$ & 1 & & & \\
\hline 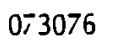 & ODHE & 20BP2-0182SAKA & 16 OENO & 7SEED & 1 & & & \\
\hline 073076 & ODHE & 20BP2-0183SAKA & 19OENO & 2 & & & & \\
\hline 073076 & ODHE & 20BP2-0184SAKA & 19OENO & 4 & & & & \\
\hline 073076 & ODHE & 20BP2-0185SAKA & T8OENO & 8ECCR & 1 & & & \\
\hline 073076 & ODHE & 20BP2-0151SAKA & I8SPHA & 11ECCR & IPOA & 1 & & \\
\hline 073076 & ODHE & $20 B P 2-0152 S A K A$ & 15SPHA & 13ECCR & $29 O A$ & 2SEED & TSHEP & 2 \\
\hline 073076 & ODHE & 20BP2-0153SAKA & 11SPHA & 16 & & & & \\
\hline 073076 & ODHE & 20BP2-0154SAKA & 13SPHA & 12ECCR & 3BROM & 1OPUN & 1 & \\
\hline 073076 & ODHE & 20BP2-0155SAKA & 9SPHA & 13ECCR & $6 P O A$ & TSHEP & 2 & \\
\hline 073076 & ODHE & 20BP2-01215OLI & 4SALX & 3SAKA & 15SEED & $2 S \mathrm{SHA}$ & 1BROM & 2 \\
\hline 073076 & ODHE & 20BP2-0122SOLI & 3SALX & TSAKA & TSSEED & $4 S P H A$ & 2BROM & 2OENO \\
\hline $0 ; 3076$ & ODHE & 20BP2-0123SOLI & TSALX & ISAKA & 14SEED & TSPHA & TBROM & 4 \\
\hline 073076 & ODHE & $20 \mathrm{BP} 2-0124 \mathrm{SOLI}$ & $4 S A L X$ & 1SAKA & 17SEED & 2SPHA & 1OENO & 1 \\
\hline 073076 & ODHE & 20BP2-0125SOLI & 3SALX & 3SAKA & 14SEED & 5BROM & 2 & \\
\hline
\end{tabular}




\begin{tabular}{|c|c|c|c|c|c|c|c|c|}
\hline 073076 & ODHE & 20BP2-0131SAKA & 12SPHA & 11ECCR & $2 \mathrm{SOLI}$ & 1SEED & 1 & \\
\hline 073076 & ODHE & 20BP2-0132SAKA & 14SPHA & 14ECCR & $25 O L I$ & 1 & & \\
\hline 073076 & ODHE & 20BP2-01335AKA & 95PHA & 16ECCR & 4BROM & 1SALX & 1 & \\
\hline 073076 & ODHE & 20BP2-0134SAKA & 13SPHA & 15ECCR & $25 O L I$ & 1SALX & 3SEED & 1 \\
\hline 073076 & ODHE & 20BP2-0135SAKA & 195PHA & 16 & SOLI & 1SALX & 2 & \\
\hline 073076 & ODHE & 20BP3-0271SAKA & 17SEED & 1OENO & 2ECCR & 1POA & 2CARE & 1 \\
\hline 073076 & ODHE & 20BP3-02725AKA & 17EQUI & 1SOLI & 1ECCR & 1POA & 1 & \\
\hline 073076 & ODHE & 20BP3-02735AKA & 18 & & ECCR & $3 P O A$ & 2 & \\
\hline 073076 & ODHE & 20BP3-02745AKA & 18 & SOLI & 1ECCR & IPOA & 1CARE & 1 \\
\hline 073076 & ODHE & 20BP3-02755AKA & 19EQUI & 1 & & & & \\
\hline 073076 & ODHE & 20BP3-0251SPHA & 5SAKA & 9SEED & $25 A L X$ & 10ECCR & 5 & \\
\hline 073076 & ODHE & 20BP3-02525PHA & 1SAKA & 11 & SALX & 15ECCR & $250 \mathrm{OL}$ & 1 \\
\hline 073076 & ODHE & $208 P 3-0253$ & SAKA & 9 & SALX & 13ECCR & 6 & \\
\hline 073076 & ODHE & 20BP3-02545PHA & 3SAKA & 8 & SALX & 12ECCR & 5 & \\
\hline 073076 & ODHE & $208 P 3-0255$ & SAKA & 6 & SALX & 13ECCR & 2CARE & 2 \\
\hline 073076 & ODHE & 20BP3-02215PHA & 17SAKA & AECCR & 4 & & & \\
\hline 073076 & ODHE & 20BP3-02225PHA & 175AKA & $1 E C C R$ & 3 & & & \\
\hline 073076 & ODHE & 20BP3-0223SPHA & 16SAKA & 7 & SETA & 1POA & 1 & \\
\hline 073076 & ODHE & 20BP3-02245PHA & 17SAKA & 6ECCR & $25 A L X$ & 2 & & \\
\hline 073076 & ODHE & 20BP3-0225SPHA & $155 \mathrm{AKA}$ & AECCR & 2 & & & \\
\hline 073076 & ODHE & 20BP3-0241SAKA & 19RAPH & 1UNKG & 1 & & & \\
\hline 073076 & ODHE & 20BP3-0242SAKA & 19SEED & 1 & & & & \\
\hline 073076 & ODHE & 20BP3-02435AKA & 19RAPH & 1UNKG & 1SEED & 1 & & \\
\hline 073076 & ODHE & 20BP3-0244SAKA & 20 & & & & & \\
\hline 073076 & ODHE & 20BP3-0245SAKA & 19RAPH & 1UNKG & 1SEED & 1CARE & 1 & \\
\hline 073076 & ODHE & 20BP3-0301SAKA & 14POA & 1LUPI & 1UNKG & 3 & & \\
\hline 073076 & ODHE & 20BP3-0302SAKA & 19UNKG & 3UNKS & 1SEED & 1 & & \\
\hline 073076 & ODHE & 20BP3-0303SAKA & 19UNKS & 15EED & 1 & & & \\
\hline 073076 & ODHE & 20BP3-0304SAKA & 19SEED & 2 & & & & \\
\hline 073076 & ODHE & 20BP3-0305SAKA & 20 & & & & & \\
\hline 073076 & ODHE & 20BP3-02115AKA & 15SALX & AECCR & 1CARE & 1 & & \\
\hline 073076 & ODHE & 20BP3-0212SAKA & 13SALX & 6ECCR & 2BROMA & IPANI & 1 & \\
\hline 073076 & ODHE & 20BP3-0213SAKA & 15SALX & 6ECCR & 4CARE & 1 & & \\
\hline 073076 & ODHE & 20BP3-0214SAKA & 14SALX & $6 \mathrm{ECCR}$ & 4 & & & \\
\hline 073076 & ODHE & 20BP3-0215SAKA & 14SALX & 2ECCR & 2CARE & $2 B R O M$ & 2 & \\
\hline 073076 & ODHE & 20BP3-0291SALX & 1SAKA & 18ECCR & 1 & & & \\
\hline 073076 & ODHE & 20BP3-0292SALX & 2SAKA & 18ECCR & 1SEED & $25 O L I$ & 1 & \\
\hline 073076 & ODHE & 20BP3-0293SALX & ISAKA & 17ECCR & 2SEED & 1CHNA & 1 & \\
\hline 073076 & ODHE & 20BE3-0294SALX & 3SAKA & 19ECCR & 1SEED & 1SPCR & 2BROM & 1 \\
\hline 073076 & ODHE & 20BP3-0295SALX & 1SAKA & 18ECCR & 1SEED & 1 & & \\
\hline 073076 & ODHE & 20BP3-0231SAKA & $205 O L 1$ & 1 & & & & \\
\hline 073076 & ODHE & 20BP3-0232SAKA & 19SALX & $25 O L I$ & 1CARE & 18ROM & 1 & \\
\hline 073076 & ODHE & 20BP3-02335AKA & $17 \mathrm{SALX}$ & 5UNKG & 1SEED & 1 & & \\
\hline 073076 & ODHE & 20BP3-02345AKA & 18SALX & TECCR & $15 O L I$ & 1SEED & 1 & \\
\hline 073076 & ODHE & 20BP3-0235SAKA & 17SALX & 7 & & & & \\
\hline
\end{tabular}




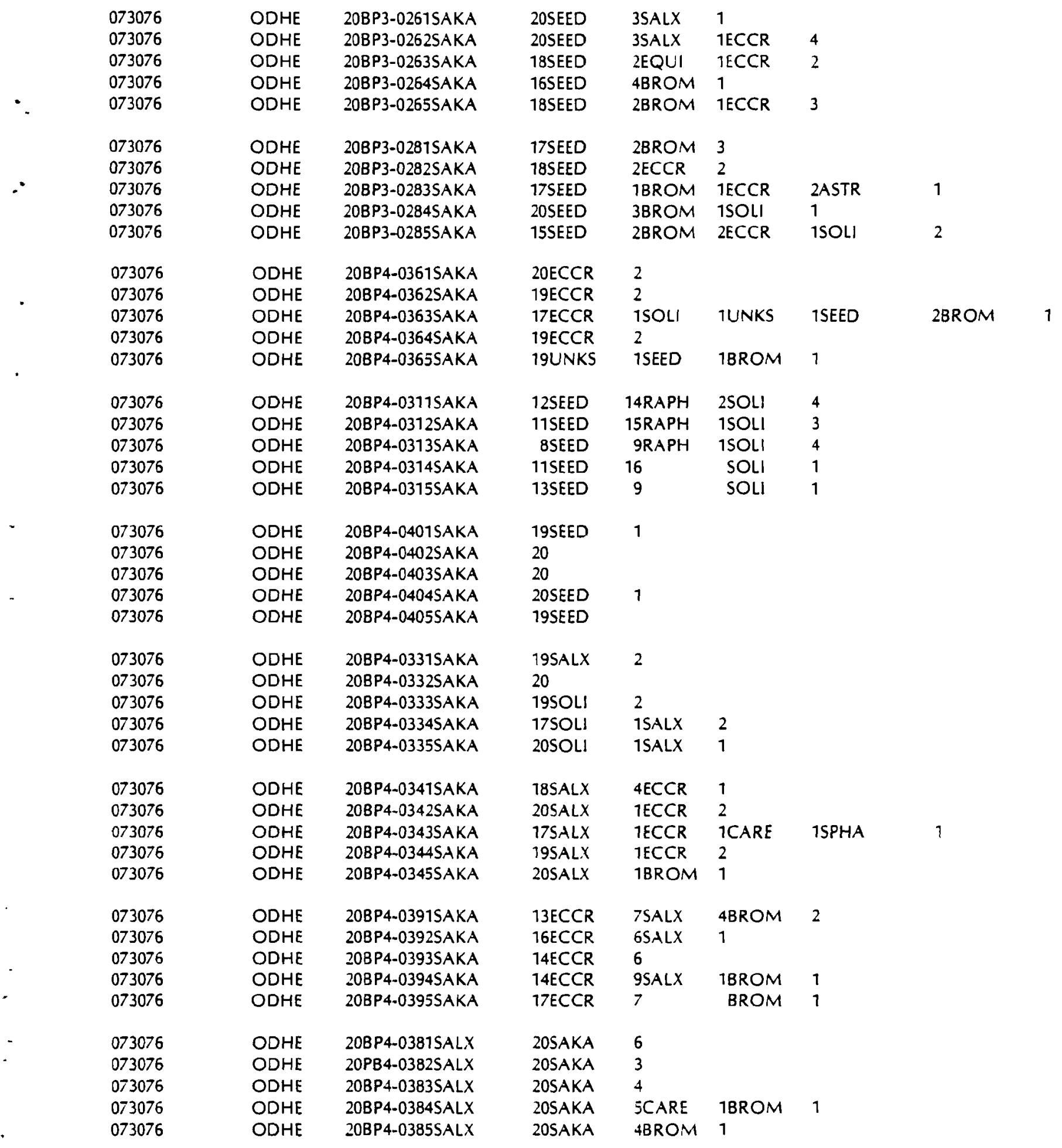




\begin{tabular}{|c|c|c|c|c|c|c|c|}
\hline 073076 & ODHE & 20BP4-0371SAKA & 18SALX & 3BROM & 1SOLI & 1 & \\
\hline 073076 & ODHE & 20BP4-0372SAKA & 19ECCR & 1BROM & 1 & & \\
\hline 073076 & ODHE & 20BP4-0373SAKA & $16 S A L X$ & 1OENO & 1 & & \\
\hline 073076 & ODHE & 20BP4-0374SAKA & 16SALX & 2BROM & 1ECCR & 2SEED & 1 \\
\hline 073076 & ODHE & 20BP4-0375SAKA & 18SALX & $250 \mathrm{OL}$ & 1 & & \\
\hline 073076 & ODHE & 20BP4-0321SAKA & 18SALX & 5 & & & \\
\hline 073076 & ODHE & 20BP4-0322SAKA & 16SALX & $6 S O L I$ & 1 & & \\
\hline 073076 & ODHE & 20BP4-0323SAKA & 20SALX & 5 & & & \\
\hline 073076 & ODHE & 20BP4-0324SAKA & 16SALX & 5 & & & \\
\hline 073076 & ODHE & 20BP4-0325SAKA & 17SALX & 4SOLI & 1BROM & 1 & \\
\hline 073076 & ODHE & 20BP4-03515AKA & 17SEED & TSALX & 1 & & \\
\hline 073076 & ODHE & 20BP4-0352SAKA & 19SEED & TSALX & 1ECCR & 1 & \\
\hline 073076 & ODHE & 20BP4-0353SAKA & 20SEED & 2 & & & \\
\hline 073076 & ODHE & 20BP4-0354SAKA & 20SEED & 1 & & & \\
\hline \multirow[t]{2}{*}{073076} & ODHE & 20BP4-0355SAKA & 20SEED & 1SALX & $2 E C C R$ & 1 & \\
\hline & \multicolumn{7}{|c|}{ BC-Cribs Site and Transect-Pellet Group No. } \\
\hline 080476 & ODHE & 20BC1-0821PUTR & BOENO & 12SALX & 5SOLI & 2 & \\
\hline 080476 & ODHE & 20BC1-0822PUTR & TOENO & 14SALX & 5SAKA & 1 & \\
\hline 080476 & ODHE & 208C1-0823PUTR & 9OENO & 14SALX & 1 & & \\
\hline 080476 & ODHE & 20BC1-0824PUTR & 10OENO & 10SALX & 2SAKA & 1 & \\
\hline 080476 & ODHE & 20BC1-0825PUTR & 12OENO & 9SALX & 2SAKA & 2 & \\
\hline 080476 & ODHE & 20BC1-0811PUTR & 13SALX & 10OENO & 3SPHA & 1 & \\
\hline 080476 & ODHE & 20BC1-0812PUTR & 17SALX & 4OENO & 3 & & \\
\hline 080476 & ODHE & 20BC1-0813PUTR & 17SALX & TOENO & 5 & & \\
\hline 080476 & ODHE & 20BC1-0814PUTR & 14SALX & TOENO & 6SPHA & 1POA & 1 \\
\hline 080476 & ODHE & 20BC1-0815PUTR & 16SALX & 6OENO & 4 & & \\
\hline 080476 & ODHE & 20BC1-0831PUTR & 20SAKA & 2 & & & \\
\hline 080476 & ODHE & 20BC1-0832PUTR & 20SAKA & 1 & & & \\
\hline 080476 & ODHE & 20BC1-0833PUTR & 20SAKA & 1ASTR & 1 & & \\
\hline 080476 & ODHE & 20BC1-0834PUTR & 2OSAKA & 1 & & & \\
\hline 080476 & ODHE & 20BC1-0835PUTR & 20ASTR & 1 & & & \\
\hline 080476 & ODHE & 20BC1-0861SALX & 20SOLI & 2 & & & \\
\hline 080476 & ODHE & 20BC1-0862SALX & 17MELI & $2 S A K A$ & 3 & & \\
\hline 080476 & ODHE & 20BC1-0863SALX & 19SOLI & 1MELI & 1 & & \\
\hline 080476 & ODHE & 20BC1-08645ALX & 20BROM & 1 & & & \\
\hline 080476 & ODHE & 20BC1-0865SALX & $20 \mathrm{SOLI}$ & 1 & & & \\
\hline 080476 & ODHE & 20BC1-0841PUTR & 20 & & & & \\
\hline 080476 & ODHE & 20BC1-0842PUTR & 20 & & & & \\
\hline 080476 & ODHE & 20BC1-0843PUTR & 20 & & & & \\
\hline 080476 & ODHE & 20BC1-0844PUTR & 20SALX & 2 & & & \\
\hline 080476 & ODHE & 20BC1-0845PUTR & $20 \mathrm{SOLI}$ & 1 & & & \\
\hline 080476 & ODHE & 20BC1-0891PUTR & 12OENO & 7SALX & 4SOI.I & 1 & \\
\hline 080476 & ODHE & 20BC1-0892PUTR & 15OENO & 4SALX & 2 & & \\
\hline 080476 & ODHE & 20BC1-0893PUTR & 13OENO & 8SALX & $1 \mathrm{SOLI}$ & 1 & \\
\hline 080476 & ODHE & 208C1-0894PUTR & 15OENO & 6SALX & 1 & & \\
\hline 080476 & ODHE & 20BC1-089SPUTR & 11OENO & 9 & & & \\
\hline
\end{tabular}




\begin{tabular}{|c|c|c|c|c|c|c|c|}
\hline 080476 & ODHE & 20BC1-0881PUTR & 20SOLI & 1 & & & \\
\hline 080476 & ODHE & 20BC1-0882PUTR & 20 & & & & \\
\hline 080476 & ODHE & 20BC1-0883PUTR & $15 S O L I$ & 2OENO & 3 & & \\
\hline 080476 & ODHE & 20BC1-0884PUTR & 20SALX & 2 & & & \\
\hline 080476 & ODHE & 20BC1-0885PUTR & TISALX & 1 & & & \\
\hline 080476 & ODHE & 20BC1-0851PUTR & 20BROM & 1SAKA & 1SALX & 1 & \\
\hline 080476 & ODHE & 20BC1-0852PUTR & 20OENO & 1 & & & \\
\hline 080476 & ODHE & 20BC1-0853PUTR & 20SAKA & 1SALX & 1 & & \\
\hline 080476 & ODHE & 20BC1-0854PUTR & $20 S A L X$ & 2 & & & \\
\hline 080476 & ODHE & 20BC1-0855PUTR & 20SAKA & 1OENO & 1 & & \\
\hline 080476 & ODHE & 20BC1.0901PUTR & $20 S A L X$ & 2 & & & \\
\hline 080476 & ODHE & 20BC1-0902PUTR & 20SALX & 2 & & & \\
\hline 080476 & ODHE & 20BC1-0903PUTR & 20SALX & ISAKA & 1 & & \\
\hline 080476 & ODHE & 20BC1-0904PUTR & 20SALX & 1SAKA & 1OENO & 1 & \\
\hline 080476 & ODHE & 20BC1-0905PUTR & 20SALX & 2SAKA & 2 & & \\
\hline 080476 & ODHE & 20BC1.0871PUTR & 20 & & & & \\
\hline 080476 & ODHE & 20BC1-0872PUTR & 20BROM & 1 & & & \\
\hline 080476 & ODHE & 20BC1-0873PUTR & 20 & & & & \\
\hline 080476 & ODHE & 20BC1-0874PUTR & 20SALX & 1 & & & \\
\hline 080476 & ODHE & 20BC1-0875PUTR & 20 & & & & \\
\hline 080476 & ODHE & 20BC2-0921SALX & 8PLTR & 9SEED & 4SAKA & 2OENO & 2 \\
\hline 080476 & ODHE & $20 B C 2-0922 S A L X$ & BPUTR & 13SEED & 4SAKA & 1 & \\
\hline 080476 & ODHE & $20 B C 2-0923 S A L X$ & SPLTR & 135EED & 1OENO & $1 \mathrm{SOLI}$ & 1 \\
\hline 080476 & ODHE & 20BC2-0924SALX & 9PUTR & 12SEED & 9SAKA & TOENO & 2 \\
\hline 080476 & ODHE & 20BC2-0925SALX & 8PUTR & 14SEED & 5SAKA & 4 & \\
\hline 080476 & ODHE & 20BC2-0961PUTR & 20SALX & 2 & & & \\
\hline 080476 & ODHE & 20BC2-0962PUTR & 20SALX & 1 & & & \\
\hline 080476 & ODHE & 20BC2-0963PUTR & 20SALX & 1 & & & \\
\hline 080476 & ODHE & 20BC2-0964PUTR & 20SALX & 2 & & & \\
\hline 080476 & ODHE & 20BC2-0965PUTR & 19SALX & 2 & & & \\
\hline 080476 & ODHE & 20BC2-0941PUTR & 20OENO & 5SOLI & 1 & & \\
\hline 080476 & ODHE & 20BC2-0942PUTR & 20OENO & 6 & & & \\
\hline 080476 & ODHE & 20BC2-0943PUTR & 20OENO & 3 & & & \\
\hline 080476 & ODHE & 20BC2-0944PUTR & 20OENO & 6SAKA & 3 & & \\
\hline 080476 & ODHE & 20BC2-0945PUTR & 20OENO & SSAKA & 3 & & \\
\hline 080476 & ODHE & 20BC2-0931PUTR & ZOOENO & 4 & & & \\
\hline 080476 & ODHE & 20BC2-0932PLTR & 20OENO & 5 & & & \\
\hline 080475 & ODHE & 20BC2-0933PLTR & 20OENO & $25 \mathrm{OLI}$ & 1 & & \\
\hline $080+76$ & ODHE & 20BC2-0934PUTR & 20OENO & 45041 & 1 & & \\
\hline 080476 & ODHE & 20BC2-0935PUTR & 20OENO & 2 & & & \\
\hline 080476 & ODHE & 20BC2-0991SALX & 14MELI & SPUTR & 1SAKA & 2 & \\
\hline 080476 & ODHE & 20BC2-0992SALX & 15MELI & 5SOLI & TSTIP & 1 & \\
\hline 080476 & ODHE & 208C2-0993SALX & TAMELI & SSOLI & 2PUTR & ISAKA & 2 \\
\hline 080476 & ODHE & $20 B C 2-09945 A L X$ & 15MELI & $5 S O L I$ & IPUTR & TOENO & 1 \\
\hline 080476 & ODHE & 20BC2-0995SALX & 13MELI & $5 S O L I$ & TPUTR & 1 & \\
\hline
\end{tabular}




\begin{tabular}{|c|c|c|c|c|c|c|c|c|}
\hline 080476 & ODHE & 20BC2-0911SALX & SMELI & 6PUTR & 10VETH & 1 & & \\
\hline 080476 & ODHE & $208 C 2-0912 S A L X$ & SMELI & 6PUTR & 9SOLI & 28ROM & 1OENO & 1 \\
\hline 080476 & ODHE & $20 B C 2-09135 A L X$ & 6MELI & 4PUTR & 125011 & 1 & & \\
\hline 080476 & ODHE & 20BC2-0914SALX & 10MELI & TPUTR & 35OLI & 18ROM & 1OENO & 1SAKA 1 \\
\hline 080476 & ODHE & 20BC2-0915SALX & 4MELI & 14PUTR & 6 & & & \\
\hline 080476 & ODHE & 20BC2-1001PUTR & 20 & & & & & \\
\hline 080476 & ODHE & 20BC2-1002PUTR & $20 S A L X$ & 3 & & & & \\
\hline 080476 & ODHE & 20BC2-1003PUTR & 20SALX & 2 & & & & \\
\hline 080476 & ODHE & 20BC2-1004PUTR & $20 S A L X$ & 2 & & & & \\
\hline 080476 & ODHE & 20BC2-1005PUTR & $20 S A L X$ & 2SAKA & 2 & & & \\
\hline 080476 & ODHE & 20BC2-0971PUTR & $205 A L X$ & 4 & & & & \\
\hline 080476 & ODHE & 20BC2-0972PUTR & 20SALX & 9OENO & 2 & & & \\
\hline 080476 & ODHE & 20BC2-0973PUTR & $205 A L X$ & 5SEED & 15AKA & 3 & & \\
\hline 080476 & ODHE & 20BC2-0974PUTR & $205 A L X$ & 7SAKA & 2 & & & \\
\hline 080476 & ODHE & 20BC2-0975PUTR & 20SALX & 6 & & & & \\
\hline 080476 & ODHE & 20BC2-0951PUTR & 20SAKA & $15 O \mathrm{LI}$ & 1SALX & 1 & & \\
\hline 080476 & ODHE & 20BC2-0952PUTR & 20SAKA & 4SOLI & 1 & & & \\
\hline 080476 & ODHE & 20BC2-0953PUTR & 20SAKA & 1OENO & 1 & & & \\
\hline 080476 & ODHE & 20BC2-0954PUTR & 20SAKA & $25 O L I$ & 1 & & & \\
\hline 080476 & ODHE & 20BC2-0955PUTR & 20SAKA & 2 & & & & \\
\hline 080475 & ODHE & 20BC2-0981PUTR & 20SAKA & 1 & & & & \\
\hline 080476 & ODHE & 20BC2-0982PUTR & 20SALX & 2 & & & & \\
\hline 080476 & ODHE & 20BC2-0983PUTR & 20MELI & 2 & & & & \\
\hline 080476 & ODHE & 208C2-0984PUTR & 20MELI & 1 & & & & \\
\hline 080476 & ODHE & 20BC2-0985PUTR & 20MELI & 1SAKA & 1 & & & \\
\hline
\end{tabular}

Gable Mtn. Pond Site and Transect-Pellet Group No.

\begin{tabular}{|c|c|c|c|c|c|c|c|}
\hline 072976 & ODHE & 20CM1-0491SALX & 15MELI & 7UNKS & 1 & & \\
\hline 072976 & ODHE & 20CM1-0492SALX & 11MELI & 9SAKA & 1 & & \\
\hline 072976 & ODHE & 20CM1-0493SALX & 11MELI & 12 & & & \\
\hline 072976 & ODHE & 20CM1-0494SALX & 10MELI & 10 & & & \\
\hline 072976 & ODHE & 20CM1-0495SALX & 13MELI & 8SAKA & 1 & & \\
\hline 072976 & ODHE & 20GM1-0501MELI & 19SALX & 3BAHY & 1 & & \\
\hline 072976 & ODHE & 20GM1-0502MELI & $12 S A L X$ & 4BAHY & $15 A K A$ & $2 \mathrm{SOLI}$ & \\
\hline 072976 & ODHE & 20GM1-0503MELI & $12 S A L X$ & 5BAHY & 2SAKA & TOENO & \\
\hline 072976 & ODHE & 20GM1-0504MELI & & ZB.AHY & 1SEED & 1 & \\
\hline 072976 & ODHE & 20GM1-0505MELI & 18SALX & 1SOLI & 1 & & \\
\hline 072976 & ODHE & 20GM1-0441SOLI & 20MELI & 1 & & & \\
\hline 072976 & ODHE & 20GM1-0442SOL1 & 20MELI & 2 UNKF & 1 & & \\
\hline 072976 & ODHE & $20 \mathrm{GM} 1-0443 \mathrm{SOLI}$ & 20MELI & 4 & & & \\
\hline 072976 & ODHE & 20GM1-0444SOLI & 19MELI & 2 & & & \\
\hline 072976 & ODHE & 20GM1-0445SOLI & 20MELI & 2LNKF & 1 & & \\
\hline 072976 & ODHE & 20GM1-0471SALX & T5MELI & ABROM & $25 O L I$ & 1 & \\
\hline 072976 & $\mathrm{ODHE}$ & 20GMT-0472SALX & 14MELI & ABROM & 1 & SAKA & 1 \\
\hline 072976 & ODHE & 20GM1-0413SALX & 13MELI & 3BROM & $25 O L I$ & 3SAKA & 3 \\
\hline 072976 & ODHE & 20GM1-0474SALX & 10MELI & SBROM & $35 O L I$ & ISAKA & 2 \\
\hline 072976 & ODHE & 20GM7-0475SALX & 11MELI & 5BROM & 3SOLI & 1 & \\
\hline
\end{tabular}




\begin{tabular}{|c|c|c|c|c|c|c|c|c|c|}
\hline & 072976 & ODHE & $20 \mathrm{CM} 1-04315 \mathrm{SII}$ & 10UNKF & 1SAKA & 1LACT & 1BROM & 1EQUI & 1 \\
\hline & 072976 & ODHE & $20 \mathrm{CM} 1-04325 \mathrm{OLI}$ & 15UNKF & 4SAKA & 1MELI & 1SPHA & 1 & \\
\hline & 072976 & ODHE & 20GM1-04335OLI & 20BROM & 1 & & & & \\
\hline & 072976 & ODHE & 20CM1-0434SOLI & 17 UNKF & 4SAKA & 1MELI & 1EQUI & 1 & \\
\hline & 072976 & ODHE & 20GM1-0435SOLI & $16 U N K F$ & 3SAKA & ILACT & 1 & & \\
\hline & 072976 & ODHE & $20 \mathrm{GM} 1-0481 \mathrm{MELI}$ & 6LACT & $35 O L I$ & 13EQUI & 3BROM & 1SALX & 1 \\
\hline & 072976 & ODHE & $20 \mathrm{CM} 1-0482 \mathrm{MELI}$ & $13 \mathrm{~L}, \mathrm{ACT}$ & $35 O L I$ & 13EQUI & 1CARE & 2 & \\
\hline - & 072976 & ODHE & 20GM1-0483MELI & 6LACT & $7 S O L I$ & 11EQUI & 3BROM & 1SALX & 1ORHY \\
\hline & 072976 & ODHE & 20GM1-0484MELI & 8LACT & $35 O L I$ & TSALX & 1CARE & 2 & \\
\hline & 072976 & ODHE & $20 \mathrm{GM} 11-0485 \mathrm{MELI}$ & SLACT & +SOLI & 10CARE & 1 & & \\
\hline & 072976 & ODHE & 20GM1-04215OLI & 20MELI & 3LACT & 1 & & & \\
\hline & 072976 & ODHE & $20 \mathrm{CM} 7-04225 \mathrm{OLI}$ & 14MELI & 4LACT & $2 \mathrm{RAPH}$ & 1BAHY & 1 & \\
\hline & 072976 & ODHE & $20 \mathrm{GM} 1-04235 \mathrm{OLI}$ & T9MELI & 1 & & & & \\
\hline & 072976 & ODHE & 20GM1-04245OLI & 16MELI & 6BAHY & 1 & & & \\
\hline & 072976 & ODHE & 20GM1-0425SOLI & 19MEL! & 2RAPH & 1 & & & \\
\hline & 072976 & ODHE & 20GM1-0461SOLI & 18MELI & $\triangle E Q U I$ & 3LACT & 1 & & \\
\hline & 072976 & ODHE & $20 \mathrm{GM} 1-04625 \mathrm{OLI}$ & 15MELI & TEQUI & 2LACT & 4 & & \\
\hline & 072976 & ODHE & $20 \mathrm{GM} 1-04635 \mathrm{OLI}$ & 19MELI & 1EQUI & 2LACT & 4ORHY & 1 & \\
\hline & 072976 & ODHE & $20 \mathrm{GM} 1-04645 \mathrm{OLI}$ & 18MELI & 3EQUI & 2LACT & 2 & & \\
\hline & 072976 & ODHE & $20 \mathrm{GM} 1-0465 \mathrm{SOLI}$ & 20 & EQUI & 1LACT & 2 & & \\
\hline & 072976 & ODHE & 20GM1-0451SALX & 13BAHY & 8MELI & $75 O L I$ & 3 & & \\
\hline & 072976 & $\mathrm{ODHE}$ & $20 \mathrm{GM} 1-0452 \mathrm{SALX}$ & $12 \mathrm{BAHY}$ & 15MELI & $45 O L I$ & 2MUHL & 1 & \\
\hline & 072976 & ODHE & 20GM1-0453SALX & $12 \mathrm{BAHY}$ & TOMELI & 4 & BROM & 3 & \\
\hline & 072976 & ODHE & 20CM7-04545ALX & 17BAHY & 7MELI & 1 & & & \\
\hline & 072976 & ODHE & 20CM1-0455SALX & 5BAHY & 9MEL! & 6 & & & \\
\hline & 073076 & ODHE & $20 \mathrm{CM} 1-04715 \mathrm{OLI}$ & 4MELI & 9BROM & 1LACT & 3EQUI & 2BAHY & 5SALX \\
\hline & 073076 & ODHE & $20 \mathrm{GM} 1-0471 \mathrm{CHNA}$ & 1 & & & & & \\
\hline & 073076 & ODHE & $20 \mathrm{GM} 1-04725 \mathrm{OLI}$ & $3 M E L I$ & 9LACT & 5BAHY & 5SALX & 1 & \\
\hline & 073076 & ODHE & $20 \mathrm{GM} 1-04735 \mathrm{OLI}$ & 6MALL & ALACT & उBAHY & TSALX & 15AKA & 1SPHA \\
\hline & 073076 & $\mathrm{ODHE}$ & $20 \mathrm{GM} 1-04745 \mathrm{OLI}$ & 8MELI & GLACT & 3BAHY & 5SALX & 2SAKA & 1EQUI \\
\hline & 073076 & ODHE & 20GM1-0474BROM & 1 & & & & & \\
\hline & 073076 & ODHE & 20CM1-0475SOLI & 4MELI & 6LACT & 5BAHY & 3SALX & 1SAKA & 2ORYZ \\
\hline & 072976 & ODHE & 20GM2-0561MELI & 18SALX & 14PHLO & 1 & & & \\
\hline & 072976 & ODHE & 20CM2-0562MELI & 2OSALX & 13PHLO & IUNKF & 1 & & \\
\hline & 072976 & ODHE & 20GM2-0563MELI & 18SALX & 11PHLO & 3TYPH & 1 & & \\
\hline & 072976 & $\mathrm{OCHE}$ & 20GM2-0564MELI & 18SALX & 14PHLO & 1TYPH & 1 & & \\
\hline & 072976 & ODHE & 20CM2-0565MELI & 20SALX & 13PHLO & 2BROM & 1 & & \\
\hline & 072976 & ODHE & 20GM2-0531MELI & $105 O L 1$ & 12ANDR & 6BAHY & 2 & & \\
\hline & 072975 & ODHE & $20 \mathrm{CM} 2-0532 \mathrm{MEL}$ & 10 SOLI & 9ANDR & 5UNKS & 2LACT & 1BROM & $2 S A L X$ \\
\hline & 072976 & ODHE & 20GM2-0533MELI & $5 S O L I$ & 11ANDR & TBAHY & 1UNKS & 1SALX & 4SEED \\
\hline & 072976 & $\mathrm{ODHE}$ & $20 \mathrm{GM} 2-0534 \mathrm{MELI}$ & $105 O L I$ & 9ANDR & 2UNKS & 3SALX & $2 \mathrm{POA}$ & 1 \\
\hline . & 072976 & ODHE & 20CM2-0535MELJ & $115 \mathrm{OLI}$ & 5ANDR & 3UNKS & 2SALX & 8LESQ & 1 \\
\hline & 072976 & ODHE & 20GM2-0591PLTR & 18SEED & 1:MELI & 1 & & & \\
\hline & 072976 & ODHE & 20GM2-0592PUTR & 17SEED & 3MEL! & $25 O L I$ & 1LACT & 1 & \\
\hline & 072976 & ODHE & 20GM2-0593PUTR & 18SEED & 1 & SOLI & 2SPHA & 1 & \\
\hline & 072976 & ODHE & 20GM2-0594PUTR & 15SEED & 1 & SOLI & 2 & & \\
\hline & 072976 & $\mathrm{ODHE}$ & 20CM2-0595PUTR & 18SEED & 1MEL! & $150 L 1$ & TLNKF & 1 & \\
\hline
\end{tabular}




\begin{tabular}{|c|c|c|c|c|c|c|c|c|c|}
\hline 072976 & ODHE & 20GM2-0521SOLI & 16SAKA & 2SEED & 2AGRO & 18ROM & 1MELI & 2 & \\
\hline 072976 & ODHE & $20 \mathrm{CM} 2-05225 \mathrm{OLI}$ & 13SAKA & 2SEED & 5BAHY & 2 & & & \\
\hline 072976 & ODHE & 20GM2-0523SOLI & 15SAKA & 2SEED & 4BROM & 1LACT & 1 & & \\
\hline 072976 & ODHE & 20GM2-0524SOLI & 15SAKA & 3SEED & 4ACRO & 1 & & & \\
\hline 072976 & ODHE & 20GM2-0525SOLI & 13SAKA & 4SEED & $2 \mathrm{MELI}$ & 2BROM & 2 & & \\
\hline 072976 & ODHE & 20GM2-0551MELI & 19SALX & 18 & & & & & \\
\hline 072976 & ODHE & 20CM2-0552MELI & 20SALX & 17CARE & 1 & & & & \\
\hline 072976 & ODHE & 20GM2-0553MELI & 20SALX & 16 & & & & & \\
\hline 072976 & ODHE & 20GM2-0554MELI & 18SALX & 168ROM & 1SAKA & 1 & & & \\
\hline 072976 & ODHE & 20GM2-0555MELI & 19SALX & 16 & & & & & \\
\hline 072976 & ODHE & 20GM2-0511SOLI & 20 & & & & & & \\
\hline 072976 & ODHE & $20 \mathrm{GM} 2-0512 \mathrm{SOLI}$ & 20 & & & & & & \\
\hline 072976 & ODHE & 20CM2-05135OI.I & 20 & & & & & & \\
\hline 072976 & ODHE & $20 \mathrm{CM} 2-05145 \mathrm{OLI}$ & 20 & & & & & & \\
\hline 072976 & ODHE & $20 \mathrm{CM} 2-05155 \mathrm{OLI}$ & 20BROM & 1 & & & & & \\
\hline 072976 & ODHE & 20GM2-05415OLI & 9MELI & BSALX & $8 \mathrm{LACT}$ & 1SPHA & 1BROM & 1 & \\
\hline 072976 & ODHE & 20GM2-0542SOLI & 6MEI.! & 14SALX & 7LACT & 1 & & & \\
\hline 072976 & ODHE & $20 \mathrm{CM} 2-0543 \mathrm{SOLI}$ & $6 \mathrm{MELI}$ & 6SALX & 11LACT & 1SPHA & 2 & & \\
\hline 072976 & ODHE & $20 \mathrm{GM} 2-05445 \mathrm{OLI}$ & 6MELI & 10SALX & 8CARE & 1 & & & \\
\hline 072976 & ODHE & $20 \mathrm{CM} 2.0545 \mathrm{SOLI}$ & 6MELI & 6SALX & 10LACT & $2 \mathrm{SPHA}$ & 4 & & \\
\hline 072976 & ODHE & $20 \mathrm{CM} 2-0581 \mathrm{MEL}$. & 1SOLI & 18SALX & 1 & & & & \\
\hline 072976 & ODHE & 20CM2-0582MELI & 4SOLI & 13SALX & 3 & & & & \\
\hline 072976 & ODHE & 20GM2-0583MELI & 2SOLI & 13SALX & $3 \mathrm{LACT}$ & 2SPHA & 1 & & \\
\hline 072976 & ODHE & 20СM2-0584MELI & 5SOLI & 13SALX & 4SPHA & 1 & & & \\
\hline 072976 & ODHE & 20CM2-0585MELI & $1 \mathrm{SOLI}$ & 12 SALX & 3LACT & ISPHA & 1OENO & 1ARIS & 1 \\
\hline 072976 & ODHE & 20GM2-0601MEI.I & 3SALX & 5SOLI & 13SEED & 2 & & & \\
\hline 072976 & ODHE & 20CM2-0602MELI & 4SALX & $3 \mathrm{SOLI}$ & 9SEED & 2SPHA & $1 \mathrm{LACT}$ & 2BAHY & 1 \\
\hline 072976 & ODHE & 20GM2-0603MELI & TSALX & $2 \mathrm{SOLI}$ & 8SEED & 1EQUI & 1 & & \\
\hline 072976 & ODHE & 20GM2-0604MELI & $3 S A L X$ & 3SOLI & 11SEED & 2LACT & 1 & & \\
\hline 072976 & ODHE & 20GM2-0605MELI & 4 & SOLI & 14LACT & 3 & & & \\
\hline 072976 & ODHE & 20GM2-0571SALX & 10SOLI & 5ASTR & 10LACT & 1 & & & \\
\hline 072976 & ODHE & 20GM2-0572SALX & 11SOLI & 4ASTR & 18SEED & 2 & & & \\
\hline 072976 & ODHE & 20GM2-0573SALX & $12 \mathrm{SOLI}$ & 5ASTR & 11SEED & 3 & & & \\
\hline 072976 & ODHE & 20GM2-0574SALX & BSOLI & 3ASTR & 12SEED & 2LACT & 1MELI & 1 & \\
\hline 072976 & ODHE & 20GM2-0575SALX & 9SOLI & 5ASTR & 15SEED & 2MELI & 1 & & \\
\hline 073076 & ODHE & 20GM3-0681SALX & 14MELI & 5SPHA & 3BROM & 1 & & & \\
\hline 073076 & ODHE & 20CM3-0682SALX & 14MELI & 9SPHA & 3 & & & & \\
\hline 073076 & ODHE & 20GM3-0683SALX & 14MELI & 8SPHA & 4SOLI & 2EQUI & 1 & & \\
\hline 073076 & ODHE & 20GM3-0684SALX & 12MELI & 6SPHA & 7BROM & 1SOLI & 1 & & \\
\hline 073076 & ODHE & 20GM3-0685SALX & 15MELI & TSPHA & 1BROM & 1 & & & \\
\hline 073076 & ODHE & 20GM3-0631SOLI & 8MELI & 3SAKA & 10 & & & & \\
\hline 073076 & ODHE & $20 \mathrm{GM} 3-0632 \mathrm{SOLI}$ & 7MELI & 1SAKA & 15SALX & 1 & & & \\
\hline 073076 & ODHE & 20GM3-0633SOLI & 6MELI & 3SAKA & 12 & & & & \\
\hline 073076 & ODHE & 20GM3-0634SOLI & 5MELI & 1SAKA & 17SALX & 2BAHY & 2 & & \\
\hline 073076 & ODHE & 20GM3-0635SOLI & 3MELI & 1SAKA & 16SALX & 2 & & & \\
\hline
\end{tabular}




\begin{tabular}{|c|c|c|c|c|c|c|c|c|c|c|}
\hline & 073076 & ODHE & $20 \mathrm{CM} 3-0671 \mathrm{MELI}$ & $35 O L I$ & 13SAKA & 3SEED & 1 & & & \\
\hline & 073076 & ODHE & $20 \mathrm{CM} 3-0672 \mathrm{MELI}$ & $4 \mathrm{SOLI}$ & 15SAKA & 1BROM & 2 & & & \\
\hline & 073076 & ODHE & 20CM3-0673MELI & 5SOLI & 14SAKA & 2BROM & 1 & & & \\
\hline & 073076 & $\mathrm{ODHE}$ & 20CM3-0674MELI & $2 S O L 1$ & 15SAKA & 1BROM & 2 & & & \\
\hline . & 073076 & ODHE & 20GM3-0675MELI & 1SOLI & 14SAKA & 1SEED & 2BROM & 1ARTE & 1 & \\
\hline & 073076 & ODHE & $20 \mathrm{GM} 3-0641 \mathrm{BROM}$ & $125 O L I$ & 9EQUI & 5SEED & 1 & & & \\
\hline & 073076 & ODHE & $20 \mathrm{CM} 3-06428 R O M$ & $5 S O L$ & 7EQUI & 6SEED & 1LUPI & 1AGRO & 3 & \\
\hline$\therefore$ & 073076 & ODHE & 20CM3-0643BROM & $7 \mathrm{SOLI}$ & TOEQUI & 8SEED & 1 & & & \\
\hline & 073076 & ODHE & $20 \mathrm{CM} 3-0644 \mathrm{BROM}$ & $6 S O L I$ & 7EQUI & 11SEED & 1AGRO & 1ARTE & 1MELI & 1 \\
\hline & 073076 & ODHE & 20GM3-0645BROM & $45 O L I$ & 7EQUI & 9SEED & 1 & & & \\
\hline & 073076 & ODHE & 20CM3-0697SOLI & 20BAHY & $1 \mathrm{LACT}$ & IRAPH & 1 & & & \\
\hline . & 073076 & ODHE & 20GM3-06925OLI & 14BAHY & 5MELI & ILACT & 1BROM & TSALX & 1 & \\
\hline & 073076 & ODHE & 20GM3-0693SOLI & 20BAHY & 2MELI & 2RAPH & 1 & & & \\
\hline & 073076 & ODHE & 20GM3-0694SOLI & 20BAHY & 3MELI & 1 & & & & \\
\hline. & 073076 & ODHE & 20GM3-0695SOLI & 17BAHY & 3MELI & 1SPHA & 1 & & & \\
\hline & 073076 & ODHE & $20 \mathrm{GM} 3-0611 \mathrm{SOLI}$ & 7SALX & 8MELI & 11BROM & 1 & & & \\
\hline & 073076 & $\mathrm{ODHE}$ & 20GM3-0612SOLI & BSALX & 7MELI & 9BAHY & 3EQUI & 1 & & \\
\hline & 073076 & ODHE & $20 \mathrm{GM} 3-06135 \mathrm{OLI}$ & 6SALX & GMELI & 9BAHY & 9SAKA & 1 & & \\
\hline & 073076 & ODHE & $20 \mathrm{GM} 3-06145 \mathrm{OLI}$ & 3SALX & 8MELI & BBAHY & GBROM & 1 & & \\
\hline & 073076 & ODHE & 20GM3-06155OLI & 6SALX & 12MELI & 11BAHY & 3BROM & $35 A K A$ & 1 & \\
\hline - & 073076 & ODHE & 20GM3-0651SOLI & 17BROM & 3MELI & 2 & & & & \\
\hline & 073076 & ODHE & $20 \mathrm{CM} 3-0652 \mathrm{SOLI}$ & 17PIPU & 1 & & & & & \\
\hline & 073076 & ODHE & 20GM3-0653SOLI & 17PIPU & 2MOSS & 1 & & & & \\
\hline - & 073076 & ODHE & $20 \mathrm{GM} 3-06545 \mathrm{OLI}$ & 17PIPU & $28 R O M$ & 1SAKA & 1 & & & \\
\hline & 073076 & ODHE & 20GM3-0655SOL! & 20PIPU & 1BROM & $1 \mathrm{LACT}$ & 1 & & & \\
\hline & 073076 & ODHE & 20GM3-0661SOLI & 20MEL! & 1 & & & & & \\
\hline & 073076 & ODHE & $20 \mathrm{GM} 3-06625 \mathrm{OLI}$ & 20 & & & & & & \\
\hline & 073076 & ODHE & 20CM3-0663SOLI & 18MELI & 3 & & & & & \\
\hline & 073076 & ODHE & 20GM3-0664SOLI & 20 & & & & & & \\
\hline & 073076 & ODHE & $20 \mathrm{CM} 3-0665 \mathrm{SOLI}$ & 19MELI & 18ROM & TARTE & 1 & & & \\
\hline & 073076 & ODHE & 20CM3-0701SALX & 16MEL! & $2 \mathrm{SOLI}$ & $2 S I A L$ & 1 & & & \\
\hline & 073076 & ODHE & 20CM3-0702SALX & 16MELI & $1 \mathrm{SOLI}$ & 4 & & & & \\
\hline & 073076 & ODHE & 20CM3-07035ALX & $16 \mathrm{MELI}$ & $15 O L 1$ & 5 & & & & \\
\hline & 073076 & ODHE & 20CM3-0704SALX & 18MELI & 1SOLI & AOENO & TBAHY & 1 & & \\
\hline & 073076 & ODHE & 20CM3-0705SALX & $16 \mathrm{MELI}$ & $25 \mathrm{SLI}$ & 6OENO & 1BROM & ISAKA & 1 & \\
\hline 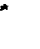 & 073076 & ODHE & 20CM3-0621MEL! & 10SALX & 1SOLI & 2BAHY & 6SAKA & 1BROM & 1 & \\
\hline & 073076 & ODHE & 20CM3-0622MELI & 5SALX & 3SOLI & 4BAHY & 9SAKA & $1 \mathrm{LACT}$ & 1 & \\
\hline - & 073076 & ODHE & 20CMM-0623MELI & SSALX & 6SOLI & 6BAHY & 5 & & & \\
\hline & 073076 & ODHE & $20 \mathrm{CM} 3-0624 \mathrm{MELI}$ & $115 A L X$ & 3SOLI & 2BAHY & 5SAKA & 3BROM & 2 & \\
\hline - & 073076 & ODHE & 20C.M3-0625MELI & 14SALX & $25 O L I$ & 3BAHY & 5 & & & \\
\hline - & 073076 & OOHE & 20CM4-0797SALX & 20MELI & 2 & & & & & \\
\hline & 073076 & ODHE & 20CM4-0792SALX & 20BROM & 2SPHA & 1 & & & & \\
\hline & 073076 & ODHE & $20 \mathrm{CM} 4-0793 \mathrm{SALX}$ & 20MELI & 1 & & & & & \\
\hline & 073076 & ODHE & 20GM4-0794SALX & $20 \mathrm{MELI}$ & 1 & & & & & \\
\hline . & 073076 & ODHE & 20GM4-0795SALX & 20MELI & 1BROM & 1EQUI & 1 & & & \\
\hline
\end{tabular}




\begin{tabular}{|c|c|c|c|c|c|c|c|c|}
\hline 073076 & ODHE & 20GM4-0751MELI & 3SOLI & 13SALX & 1SAKA & 3BROM & 2 & \\
\hline 073076 & ODHE & 20GM4-0752MELI & 4SOLI & 13BROM & $2 U N K G$ & 1BAHY & 1 & \\
\hline 073076 & ODHE & 20CM4-0753MELI & 3SOLI & 18SAKA & 2 & & & \\
\hline 073076 & ODHE & 20CM4-0754MELI & 2SOLI & 17SAKA & $4 R O M$ & 1 & & \\
\hline 073076 & ODHE & 20GM4-0755MELI & 3SOLI & 16SAKA & 3 & & & \\
\hline 073076 & ODHE & 20GM4-0781SALX & 13SOL! & 6BROM & 1 & & & \\
\hline 073076 & ODHE & 20GM4-0782SALX & 14SOLI & 4BROM & 1MELI & 4PHLO & 1 & \\
\hline 073076 & ODHE & 20GM4-0783SALX & $1350 \mathrm{LI}$ & $38 R O M$ & 1MELI & 3 & & \\
\hline 073076 & ODHE & 20GM4-0784SALX & 20SOLI & 1 & & & & \\
\hline 073076 & ODHE & 20GM4-0785SALX & 11SOLI & 4BROM & 1MELI & 2PHLO & 1GRSP & 1 \\
\hline 073076 & ODHE & 20GM4-0801SOLI & 11EQUI & $2 S A L X$ & 5SAKA & 5BAHY & 2 & \\
\hline 073076 & ODHE & 20GM4-0802SOLI & 15EQUI & 1SALX & 25AKA & 4BAHY & 1MELI & 1 \\
\hline 073076 & ODHE & 20GM14-0803SOLI & 9EQUI & 3SALX & 5SAKA & 2 & MELI & 1 \\
\hline 073076 & ODHE & 20GM4-0804SOLI & 10EQUI & 1SALX & 2SAKA & 7 & MELI & 3 \\
\hline 073076 & ODHE & 20GM4-0805SOLI & 8EQUI & 3SALX & 1SAKA & 4BAHY & 1MELI & 28ROM \\
\hline 073076 & ODHE & 20GM4-0741SALX & 12BROM & 6SOLI & 4SAKA & 1MELI & 1BAHY & 3 \\
\hline 073076 & ODHE & 20GM4-0742SALX & 13BROM & 9SOLI & 3SAKA & 3MELI & 1SEED & 3 \\
\hline 073076 & ODHE & 20GM4-0743SALX & 14BROM & 6SOLI & 1SAKA & 6MELI & 1 & \\
\hline 073076 & ODHE & 20GM4-0744SALX & 11BROM & $45 O L I$ & 4SAKA & 6 & & \\
\hline 073076 & ODHE & 20GM4-0745SALX & 10BROM & 6SOLI & 2SAKA & 4SEED & 1MELI & 2 \\
\hline 073076 & ODHE & $20 \mathrm{GM} 4-107715 \mathrm{OLI}$ & 10SALX & TBAHY & 3PANI & 1BROM & 1 & \\
\hline 073076 & ODHE & 20CM4-0772SOLI & 10SALX & 9BAHY & 1MELI & 2 & & \\
\hline 073076 & ODHE & 20GM4-0773SOLI & 10SALX & 10BAHY & 1MELI & 3BROM & 1 & \\
\hline 073076 & ODHE & $20 \mathrm{GM} 4-07745 O L I$ & T1SALX & 4BAHY & TMELI & 4 & & \\
\hline 073076 & ODHE & $20 \mathrm{CM} 4-07755 \mathrm{OLI}$ & 6SALX & 9BAHY & 1MELI & 2BROM & 2 & \\
\hline 073076 & ODHE & 20GM4-0761MELI & $125 \mathrm{ALX}$ & 12 OENO & 18AHY & 1 & & \\
\hline 073076 & ODHE & 20GM4-0762MELI & TSALX & 15SOLI & 1BROM & 1CARE & 2 & \\
\hline 073076 & ODHE & 20GM4-0763MELI & 7SALX & 15SOLI & 3 MOSS & 1 & & \\
\hline 073076 & ODHE & 20CM4-0764MELI & 6SALX & $17 S O L I$ & 3BAHY & 1 & & \\
\hline 073076 & ODHE & 20GM4-0765MELI & BSALX & 13SOLI & TBAHY & 2 & & \\
\hline 073076 & ODHE & 20GM4-0721SALX & 11SPHA & 8SOLI & 2MELI & 3OENE & 1LACT & 1 \\
\hline
\end{tabular}




\section{APPENDIX C}

RADIOLOGICAL DATA

OF MULE DEER PELLET GROUPS COLLECTED AT

B-C CRIBS, B POND, GABLE MOUNTAIN POND AND DEER CREEK, PROVO, UTAH 


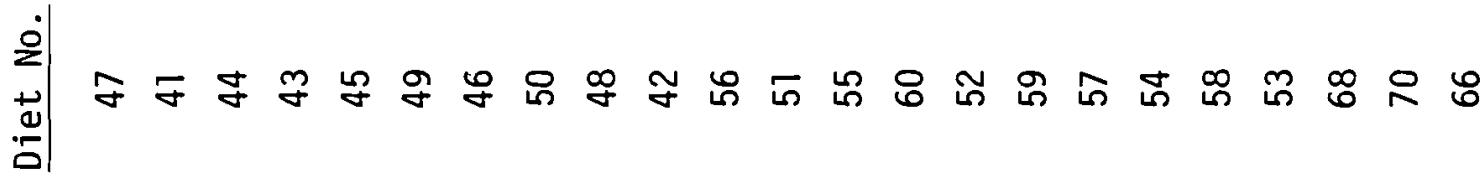

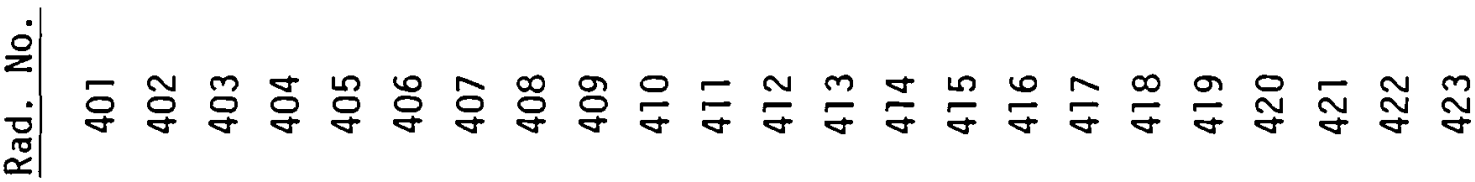

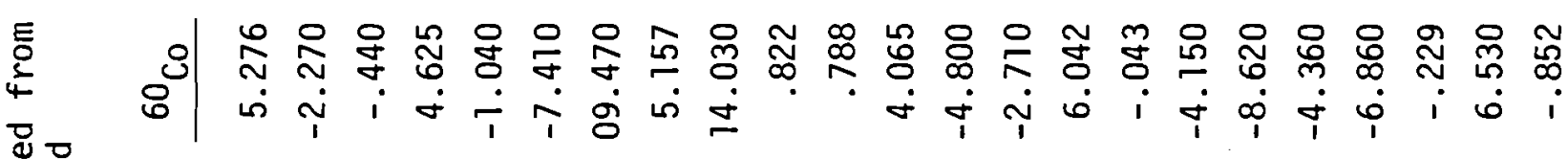
每

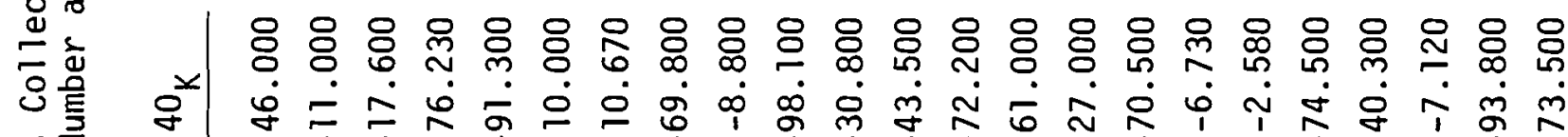

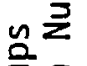

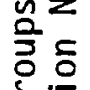

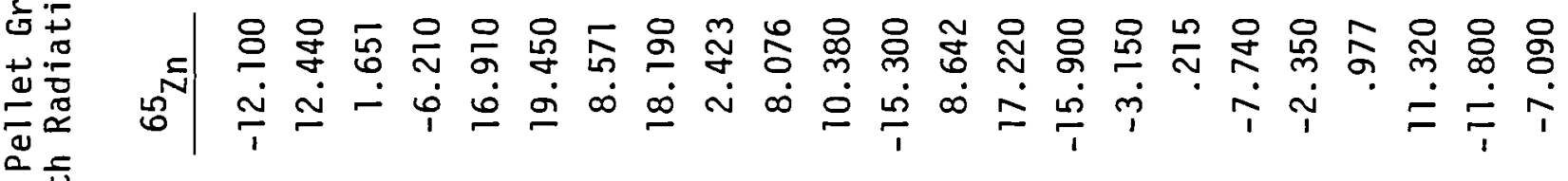

迹樆 4 +ூ

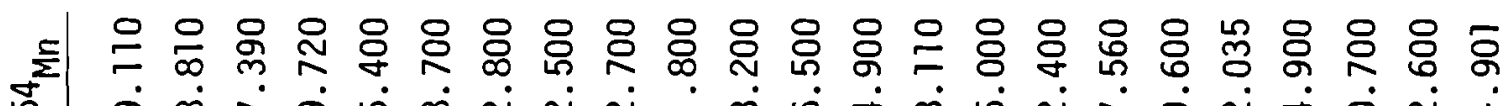
志 তิ올

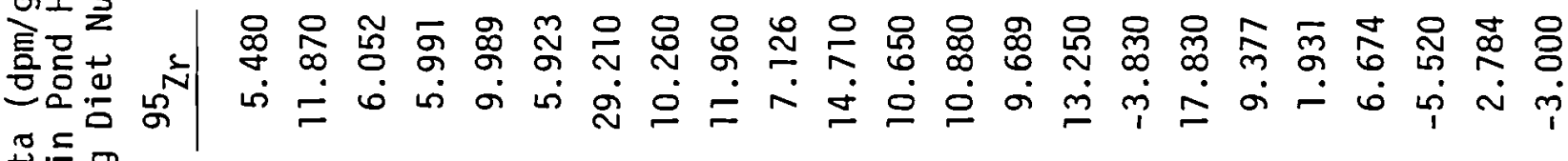
$\underset{\oplus}{\oplus}=\frac{\sigma}{\sigma}$

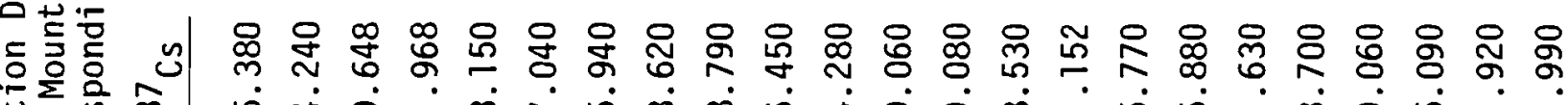

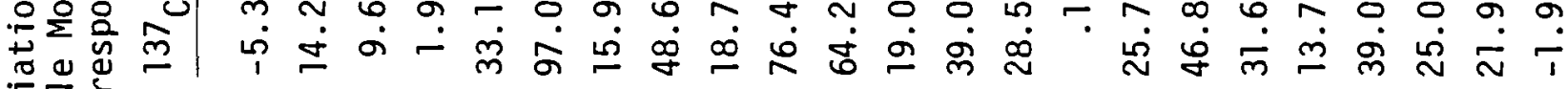

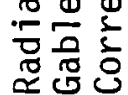

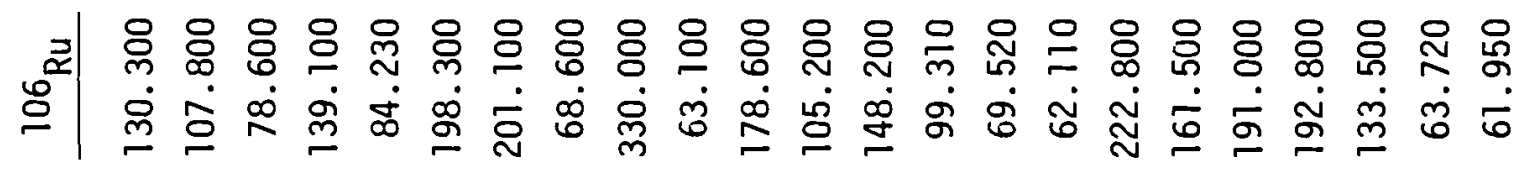

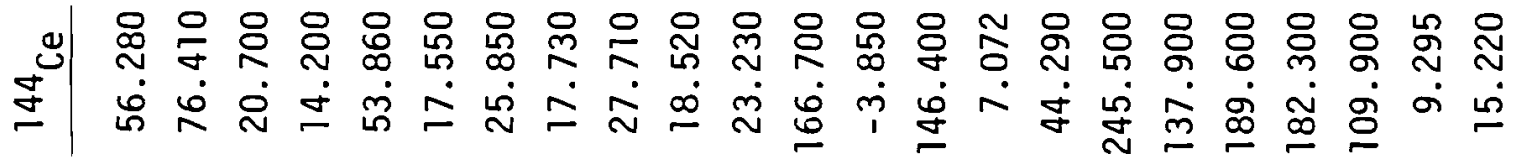


$\stackrel{\oplus}{\frac{D}{\leftrightarrows}}$

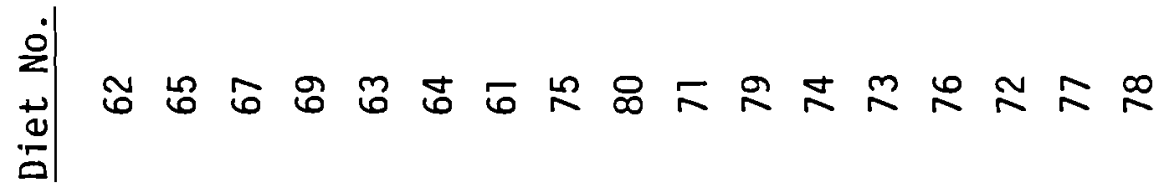

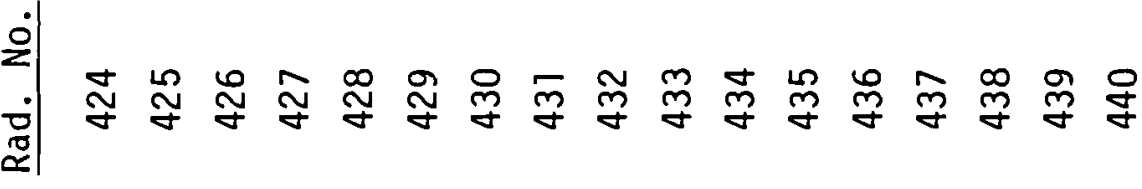

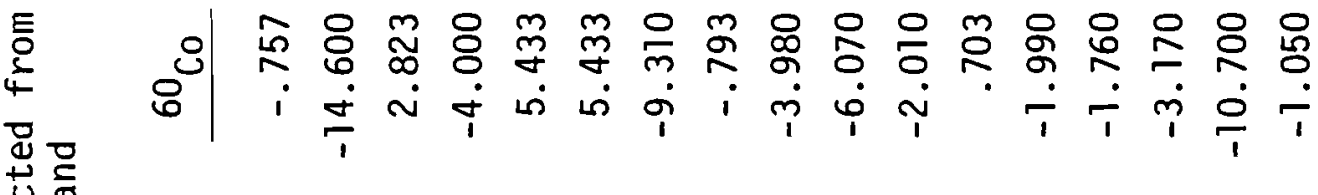

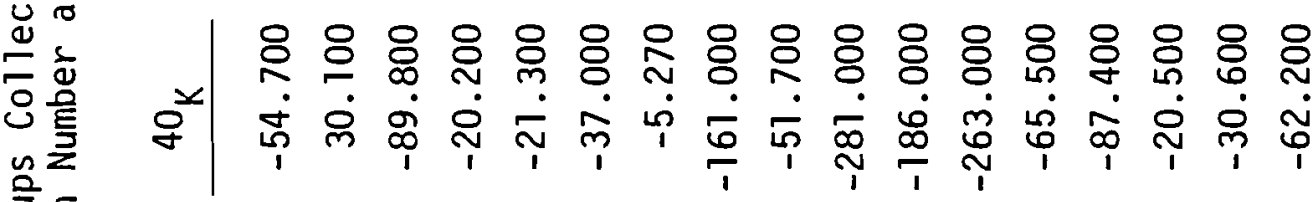
공 등

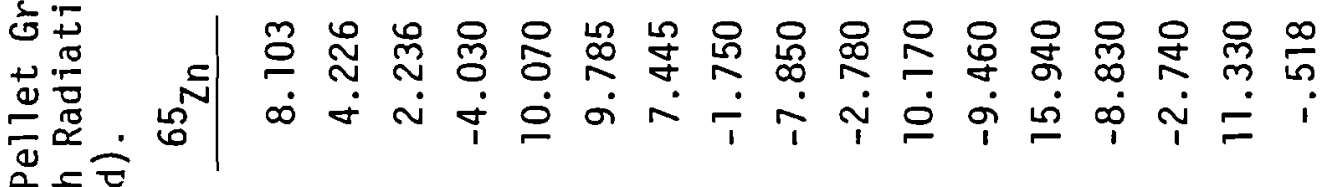
兘昰 收苜 бับ 至

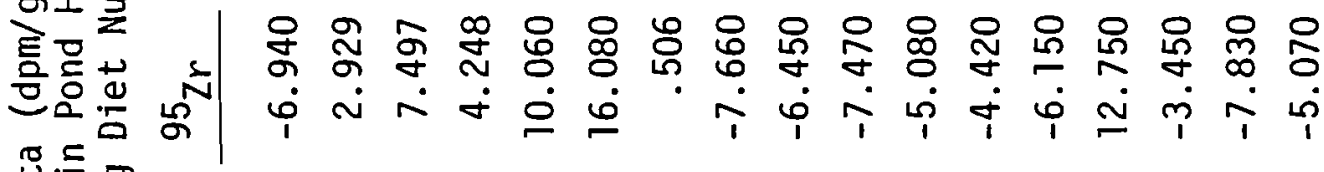
$\underset{7}{\pi} \cdot \frac{1}{8}$

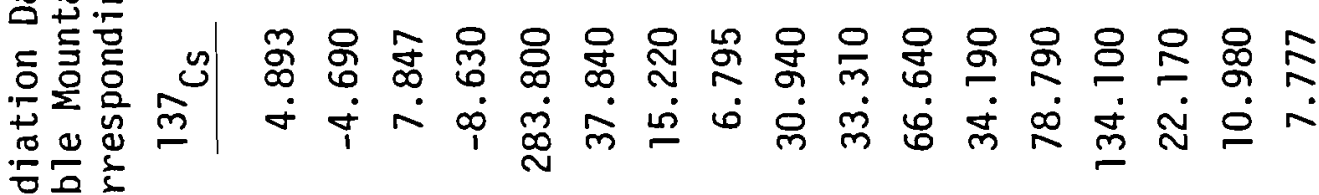

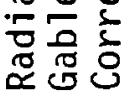

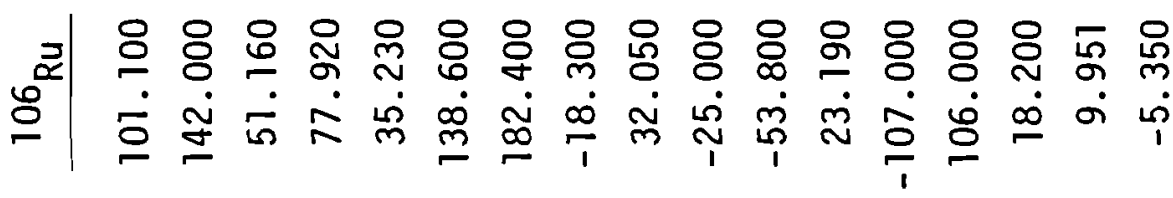

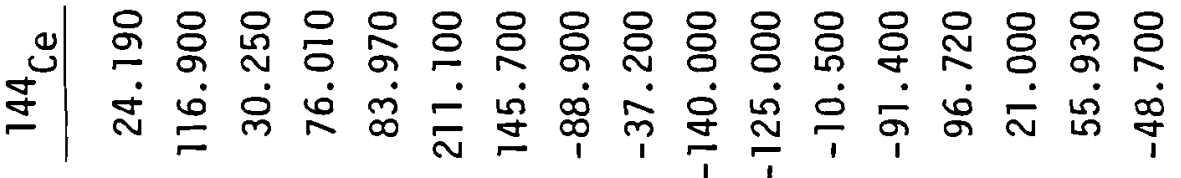


Radiation Data (dpm/g) of Deer Pellet Groups Collected from the

B-C Cribs Habitat with Radiation Number and Corresponding Diet Number.

\begin{tabular}{|c|c|c|c|c|c|c|c|c|c|}
\hline${ }^{144} \mathrm{Ce}$ & ${ }^{106} \mathrm{Ru}$ & ${ }^{137} \mathrm{Cs}$ & ${ }^{95} \mathrm{Zr}$ & ${ }^{54} \mathrm{Mn}$ & ${ }^{65} \mathrm{Zn}$ & $40_{K}$ & ${ }^{60} \mathrm{Co}$ & Rad. No. & Diet No. \\
\hline-89.500 & 40.710 & 3.961 & -1.350 & 4.376 & .819 & -70.700 & -3.630 & 441 & 87 \\
\hline 17.010 & 107.200 & 3.272 & -5.850 & -3.360 & -9.690 & -75.200 & -7.320 & 442 & 90 \\
\hline 8.567 & 44.020 & 17.710 & -5.920 & .961 & -3.170 & -39.400 & -2.330 & 443 & 84 \\
\hline 21.130 & 100.900 & 39.380 & 6.417 & 7.212 & -13.900 & -117.000 & 4.205 & 444 & 82 \\
\hline-79.900 & 53.920 & -2.230 & -8.680 & 3.208 & 11.510 & -142.000 & -9.620 & 445 & 83 \\
\hline 29.440 & 125.300 & -3.140 & -10.300 & .231 & -2.460 & -78.900 & -9.780 & 446 & 86 \\
\hline 15.230 & 84.930 & 6.800 & -7.100 & 3.502 & -24.000 & -2.750 & 3.872 & 447 & 88 \\
\hline 21.240 & 98.060 & -.682 & -.630 & -.083 & -23.300 & -67.000 & 7.073 & 448 & 81 \\
\hline 92.650 & 160.900 & -.632 & 3.470 & -5.350 & -30.900 & -1.290 & 2.226 & 449 & 89 \\
\hline 109.800 & 230.300 & -.225 & -.873 & -3.310 & -26.300 & 69.140 & -1.920 & 450 & 85 \\
\hline 58.360 & 205.700 & 1.834 & -1.920 & -3.130 & -15.800 & 47.320 & -6.020 & 451 & 94 \\
\hline 157.200 & 189.000 & -1.640 & -9.850 & -5.370 & -38.000 & 13.310 & 4.978 & 452 & 93 \\
\hline-2.890 & 88.840 & -3.340 & 1.065 & -2.870 & -11.500 & 18.550 & -1.100 & 453 & 92 \\
\hline 85.110 & 221.900 & -9.690 & -.940 & 1.303 & -38.000 & 51.200 & -3.370 & 454 & 97 \\
\hline 7.405 & 98.470 & 4.328 & 1.155 & -3.320 & -24.400 & -40.500 & 5.739 & 455 & 91 \\
\hline 107.300 & 185.000 & 1.089 & -11.500 & .280 & -27.100 & 38.900 & -1.590 & 456 & 95 \\
\hline 2.573 & 135.900 & -9.090 & -4.540 & -2.020 & -17.400 & 8.890 & 1.128 & 457 & 99 \\
\hline 106.100 & 109.800 & 5.007 & -3.190 & 2.510 & -19.500 & 3.275 & 3.435 & 458 & 100 \\
\hline 17.520 & 119.600 & 7.469 & -4.250 & 2.186 & -27.900 & -11.700 & 1.971 & 459 & 98 \\
\hline-.986 & 143.400 & 12.140 & .718 & .192 & -22.500 & -50.900 & 2.649 & 460 & 96 \\
\hline
\end{tabular}




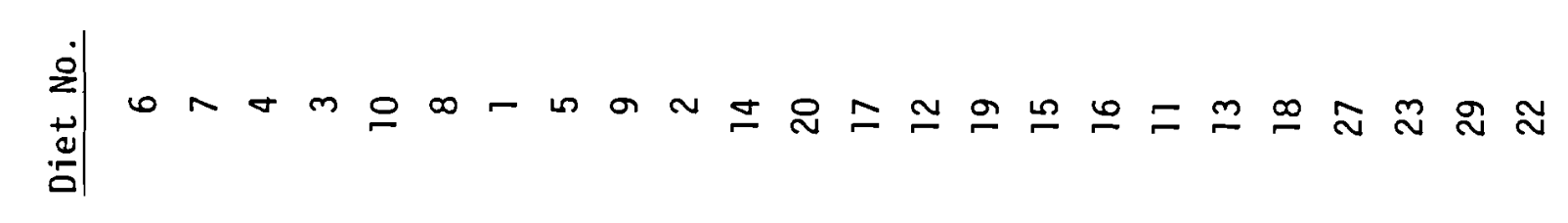

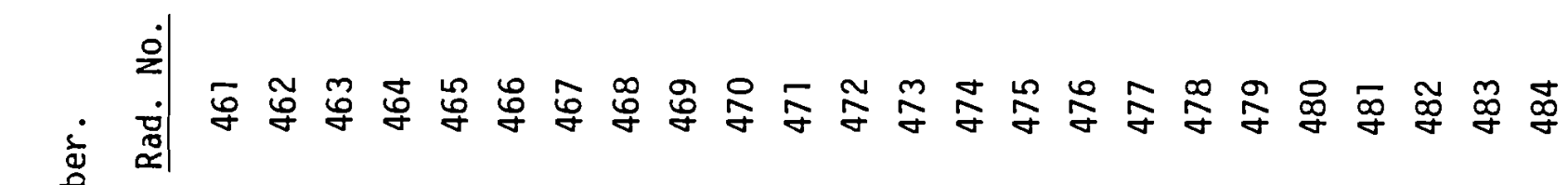

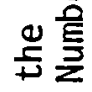

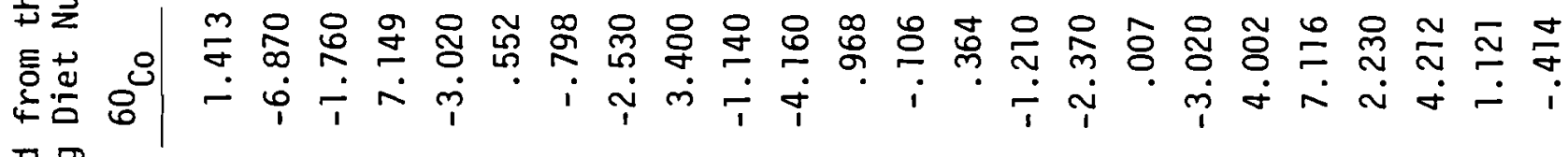
$\underset{\mathrm{d}}{\mathrm{g}} \stackrel{\mathrm{g}}{\mathrm{g}}$

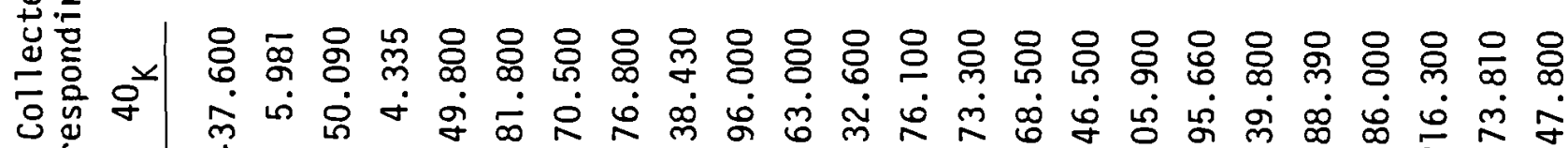
言言

)

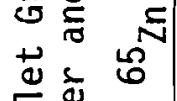

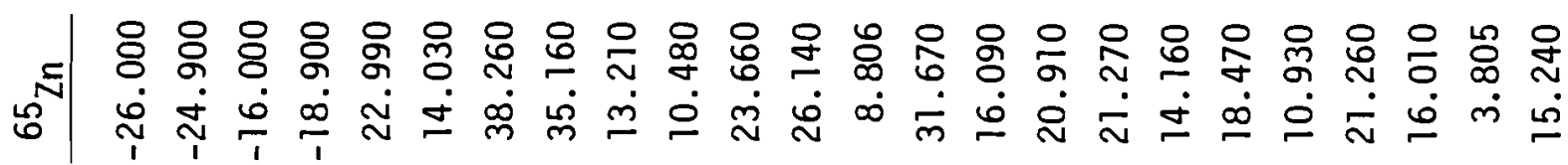
它焉

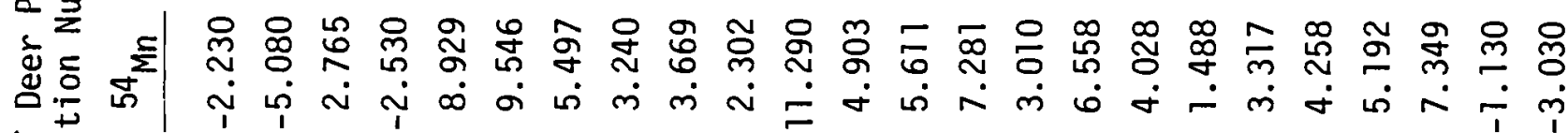

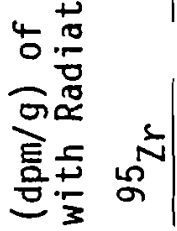

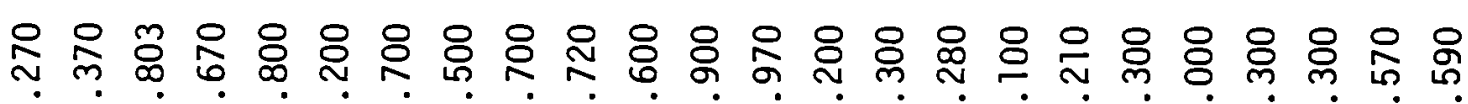
里

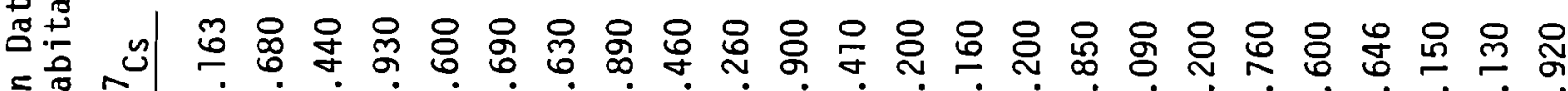
동 $+\frac{1}{0}$

동

$\approx \infty$

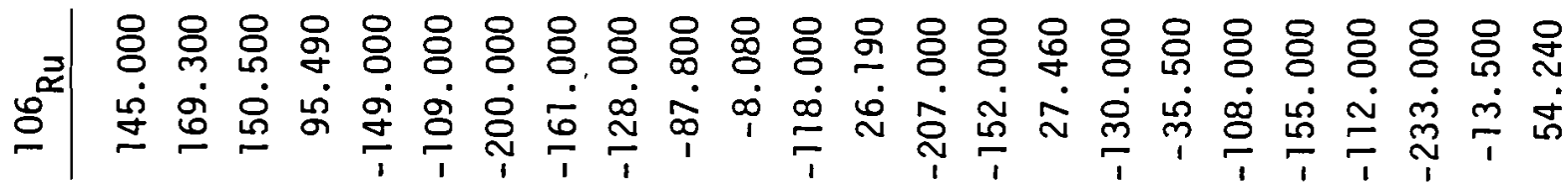

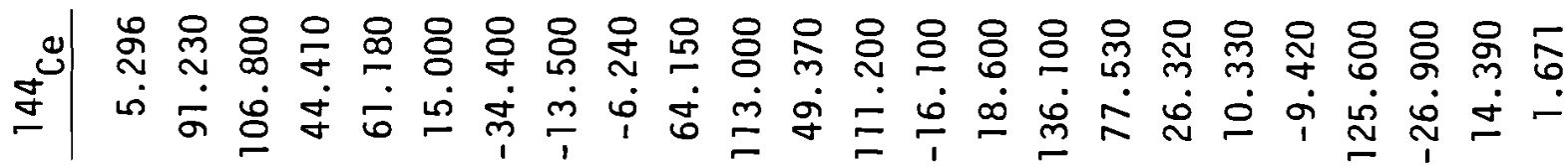


Radiation Data (dpm/g) of Deer Pellet Groups Collected from the

$B$ Pond Habitat with Radiation Number and Corresponding Diet Number (Contd).

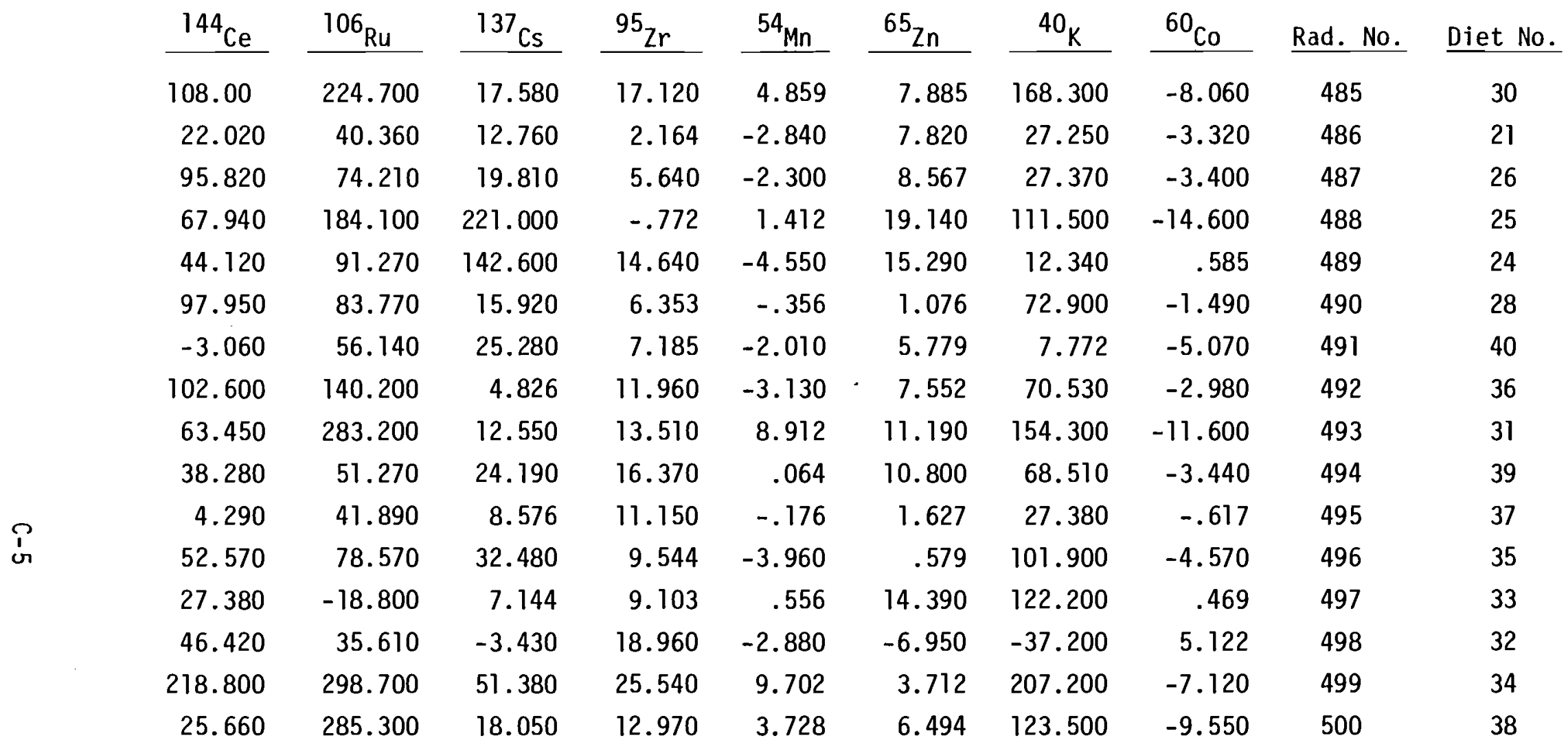




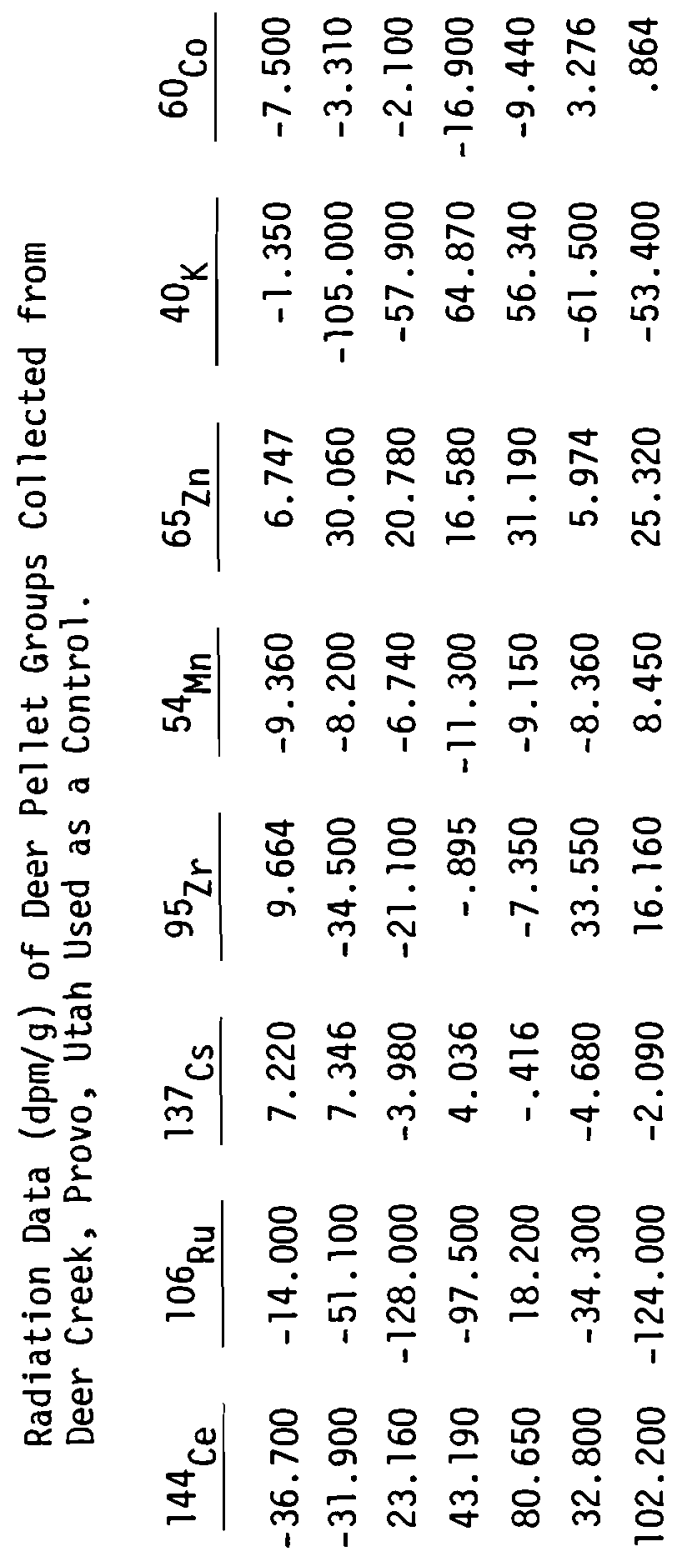




\section{OISTRIBUTION}

No. of

Copies

OFFSITE

A. A. Churm

DOE Chicago Patent Attorney

9800 South Cass Avenue

Argonne, IL 60439

27 DOE Technical Information Center

R. C. Clusen

Department of Energy

Assistant Secretary for

Environment

Washington, DC 20545

DOE Division of the Environment Washington, DC 20545

N. F. Barr

W. W. Burr

R. C. Dahlman

R. E. Franklin

W. S. Osburn

J. Swinebroad

R. L. Watters

P. B. Dunaway

DOE Nevada Operations Office

PO Box 14100

Las Vegas, NV 89114

0. D. Markham

Radiological and Environmental Sciences Laboratory

PO Box 2108

Idaho Falls, ID 83401

S. Meyers

DOE Office of Nuclear Waste

Management

Washington, DC 20545
No. of

Copies

OFFSITE

S. I. Auerbach

Oak Ridge National Laboratory

PO Box X

Oak Ridge, TN 37830

I. L. Brisbin, Jr.

Savannah River Ecology Laboratory

Aiken, SC 29801

Office of Nuclear Waste Isolation

Att: Beverly Rawles

Battelle Memorial Institute

505 King Avenue

Columbus, $\mathrm{OH} 43201$

T. E. Hakonson

Los Alamos Scientific

Laboratory

PO Box 1663

Los Alamos, NM 86544

M. Smith, Director

Savannah River Ecology Laboratory

Drawer E.

Aiken, SC 29801

F. W. Whicker

Radiology Radiation

Biology Department

Colorado State University

Fort Collins, CO 80521

United Nuclear Inc.

T. E. Dabrowski 
No. of

Copies

ONSITE

Hanford Engineering Development Laboratory

G. D. Carpenter

10 DOE - Richland Operations

R. E. (Ted) Austin/P. F. Dunigan

J. C. Cummings

D. R. Elle

B. R. Goranson

H. E. Ransom

J. L. Rhoades

J. J. Schreiber/R. E. Gerton

M. W. Shupe/M. J. Zamorski

F. R. Standerfer/P. G. Harris

M. G. White

30 Rockwel1 Hanford Operations

H. Babad

D. J. Brown

L. E. Bruns

J. L. Deichman

R. D. Fox

M. K. Harmon

W. F. Heine

R. E. Isaacson

J. E. Kinzer

W. P. Kunke 1
No. of

Copies
P. G. Lorenzini
C. W. Manry
D. A. Marsh
H. E. McGuire
R. M. Mitchell
W. L. Nees
D. Paine
J. V. Panesko
J. H. Roecker
R. E. Wheeler
D. D. Wodrich

43 Pacific Northwest Laboratory

L. L. Cadwel 1 (26)

R. E. Fitzner

K. E. Harding (4)

W. H. Rickard

N. R. Hinds

W. L. Templeton

M. L. Warner

W. R. Wiley

Technical Information (5)

Publishing Coordination (2) 